\title{
Application of the analytical hierarchy process to establish health care waste management systems that minimise infection risks in developing countries
}

\author{
Alan C. Brent ${ }^{\mathrm{a}}$, \\ David E.C. Rogers ${ }^{\mathrm{b}}$, \\ Tšaletseng S.M. Ramabitsa-Siimane ${ }^{\mathrm{a}}$ and \\ Mark B. Rohwer ${ }^{\mathrm{b}}$
}

${ }^{\mathrm{a}}$ Chair of Life Cycle Engineering, Department of Engineering and Technology Management, University of Pretoria, Lynnwood Road, 0002 Pretoria, Gauteng, South Africa

${ }^{b}$ Energy and Processes, Material Science and Manufacturing, Council for Scientific and Industrial Research, 0001 Pretoria, South Africa

Received 31 January 2006; accepted 7 June 2006. Available online 10 August 2006.

\section{Abstract}

This paper focuses on the application of the analytical hierarchy process (AHP) technique in the context of sustainable development to establish and optimise health care waste management (HCWM) systems in rural areas of developing countries. This is achieved by evaluating the way in which the AHP can best be combined with a life cycle management (LCM) approach, and addressing a main objective of HCWM systems, i.e. to minimize infection of patients and workers within the system. The modified approach was applied to two case studies: the sub-Saharan African countries of South Africa and Lesotho. Quantitative weightings from the AHP are used to identify alternative systems that have similar outcomes in meeting the systems objective, but may have different cost 
structures and infection risks. The two case studies illustrate how the AHP can be used (with strengths and weaknesses) in environmental engineering decision support in developing countries.

\section{Article Outline}

1. Introduction

1.1. Application of the AHP to sustainable development systems

1.2. Sustainable development and health care waste management (HCWM) systems

1.3. Application of the AHP as decision support for the sustainable development of health care waste management (HCWM) systems

1.4. Objectives of the research study

2. Research methodology

2.1. The representation of the AHP workshops of Southern African HCWM systems

2.2. The AHP workshops

2.2.1. Presentations

2.2.2. Open discussions

2.2.3. Consensus

2.2.4. AHP questionnaires

3. Integrating life cycle thinking to construct appropriate hierarchy trees

3.1. Waste life cycle management at primary health care facilities

3.2. Correlating AHP and waste life cycle management terminology

3.3. Structuring of hierarchical trees of options for the life cycle phases

4. Results of the workshops

4.1. Definitions of the hierarchical trees

4.2. Priority weights of the life cycle phase components and options

5. Discussion

5.1. The allocated time for the facilitation and coordination of the workshop process as a key issue in developing countries

5.2. The problem of diverse decision-makers in HCWM systems

5.3. Applying the priority weights to rank alternatives and assign risk factors

6. Conclusions and way forward

Acknowledgements

Appendix A. Hierarchical trees for the life cycle phases and research results

A.1. Acronyms of available options for the life cycle phases

References 


\section{Introduction}

This paper focuses on the application of the analytical hierarchy process (AHP) to address a specific sustainable development problem in developing countries, i.e. to minimise infection risks of health care waste management (HCWM) systems. Therefore, the AHP technique is introduced shortly with its strengths and weaknesses, and the application thereof is considered in the context of sustainable development and HCWM, to clarify the specific objectives of the study.

The AHP (Saaty, 1980 and Saaty, 1990) is a known multi-attribute weighting method for decision support (Madu, 1994). As such, the AHP has been used for solving complex decision-making problems in various disciplines, e.g. public policy (Kurttila et al., 2000), strategic planning (Bititci et al., 2001), viability determination (Alidi, 1996), forecasting (Carmone et al., 1997), and project management (Kamal, 2001). The AHP, which follows an approach of pair-wise comparison, provides a way for calibrating a numerical scale, particularly in new areas where measurements and quantitative comparisons do not exist. The process is summarised in Fig. 1.

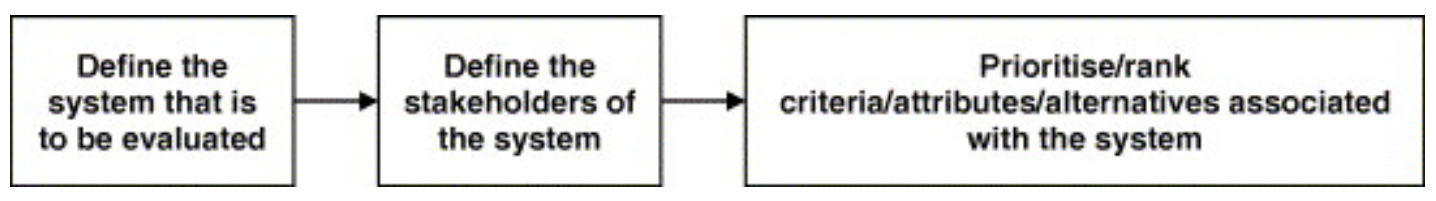

Fig. 1. A schematic diagram of the AHP process.

A number of benefits and limitations have been noted with the AHP process in general as a multi-criteria decision analysis (MCDA) technique (Morrissey and Browne, 2004):

- It allows a systematic approach to evaluate policy options and helps understanding of the problem.

- A mixture of quantitative and qualitative information can be incorporated. MCDA goes beyond the evaluation of purely economic consequences and allows non-economic 
criteria to be assessed on an equal basis, i.e. MCDA techniques offer a level of flexibility and inclusiveness that purely economic based models tend to lack.

- Account can be taken of the preferences of the various stakeholder groups with conflicting objectives (Bana et al., 1997 and Qureshi et al., 1999).

- MCDA methods do not produce the 'best' solution, but a set of preferred solutions or a general ranking of all solutions. Solving such a multi-criteria problem is, therefore, a compromise and depends on the circumstances in which the decision-aiding process is taking place.

- There is a need for personal judgement and experience in making the decisions.

- MCDA techniques are sometimes very cumbersome and unwieldy (Beynon et al., 2000).

- The allocation of weights to each criterion is subjective. Changing the weights could lead to a different result, i.e. rank reversal (Dyer, 1990).

In particular, specific limitations, associated with the application of the AHP to decisionmaking problems, have been expressed in terms of

- Its inadequacy to define complex systems, i.e. the hierarchical approach may be inappropriate for a specific system (Hokkanen and Salminen, 1997); and

- Inconsistencies ${ }^{1}$ between stakeholder prioritization of attributes for a defined system, whereby weights can be calculated. For example, individual stakeholders may be subject to judgemental errors in the pair-wise comparisons (Laininen and Hämäläinen, 2002) or may find it difficult to consider a set of pair-wise comparisons as a whole (Aguaron et al., 2003). As a result, these stakeholders may perceive intransitive relationships in the pairwise comparisons (Bodin and Gass, 2003). Alternatively, a group of stakeholders may find it difficult to reach consensus on a single or a set of pair-wise comparisons (Lai et al., 1999). Indeed, it has been argued that there is no consistency in actual choices (Hughes, 1990). 


\subsection{Application of the AHP to sustainable development systems}

The concept of sustainability and sustainable development ${ }^{2}$ can be understood intuitively, but it remains difficult to express it in concrete, operational terms (Briassoulis, 2001). However, many agree that sustainable development is about achieving environmental, economic, and social welfare for present as well as future generations (Azapagic and Perdan, 2000). From a governmental perspective this can be at national and global levels (UNCSD, 2001). From an organizational perspective this can be at a project level (Labuschagne et al., 2005a). In some cases stakeholders specifically require that environmental, economic, and social goals must be met across all levels of development. Sustainable development has subsequently been conceptualised as a state of dynamic equilibrium between societal demand for a preferred development and the supply of environmental and economic goods and services needed to meet this demand (Briassoulis, 2001). Systems approaches have been proposed to consider strategic sustainable development planning in different sectors (Robèrt et al., 2002 and Labuschagne et al., 2005b). But the intricate relationships between the three dimensions of sustainable development, i.e. environmental, economic and social welfare, have been difficult to model within the concept of a clear absolute system. Specifically, trade-offs between the three dimensions of sustainable development may not be possible to quantify as the benefits cannot be measured. Proposals for these trade-offs can be referred to as 'weak', i.e. indirectly indicating sustainability (Hanley et al., 1997, Rennings and Wiggering, 1997 and Atkinson, 2000).

Consensus on the general objectives and basic principles of sustainable development may be obtained in theory. But consensus on the details of how to achieve sustainable development or maintain sustainability is difficult to obtain in practice. This difficulty can be attributed to the variety of perceptions on specific socio-cultural and political contexts that change over time (Briassoulis, 2001 and Brent et al., 2005). For example, very high inconsistencies in the judgements of panels with regards to strategic sustainability assessments have been documented (Noble, 2004). This is most probably an inherent weakness of the AHP when it is applied without support data in the context of sustainable development. This weakness is highlighted in developing countries by the 
difficulties to establish operable systems and to obtain support data that can be measured against sustainable development criteria such as the principles proposed in Agenda $21 . \underline{3}$ Regardless, the AHP has been used before for the purposes of assessing and weighting criteria and indicators for sustainable development in specific applications (Mulder and Brent, 2006, Brent et al., 2005, Mendoza and Prabhu, 2000 and Mendoza and Prabhu, 2003).

\subsection{Sustainable development and health care waste management (HCWM) systems}

Health care is a basic need for current and future generations (UNDP, 2005), and is therefore one of the objectives of sustainable development systems. In developing countries the main symptom of poor health is the high mortality rate due to infectious diseases ${ }^{4}$ with the accompanying reduced life expectancy. For example, in Lesotho the life expectancy for women is 40 and for men 35 (WHO, 2005). The main objective identified of Health Care Waste Management (HCWM) systems in South Africa and Lesotho is to prevent the spread of infections from the health care waste to the workers and the community. This compares with the first objective of health care systems in general: "first do no harm" (Johannessen et al., 2000 and WHO, 2002). Although data on the spread of infections due to waste handling is limited, experts accept that $5 \%$ of all HIV infections in Africa are due to unsafe injections (Crabb, 2003), of which unsafe waste disposal is a component (WHO, 1999). Surveys of rural primary health care facilities indicate that needle pricks occur in the work place (Grimmond et al., 2003 and Ramabitsa-Siimane, 2006). The probability of infection due to needle prick is higher for hepatitis than for HIV (Kane et al., 1999).

The HCWM system can be seen as a sub-system of the health care system. Therefore, optimizing efficiency of the waste management system, although not a guarantee for improvement in health care systems, is a component of sustainable development in such systems.

The balancing of tradeoffs between the three dimensions of sustainable development, i.e. environment, economy, and society, may not be so difficult when a systems approach is 
taken to HCWM. Therefore, it can be argued that "strong sustainability" criteria can be identified for waste management systems, i.e. a direct relationship between action and sustainability response.

Health care systems can be described quantitatively using standardized systems tools, such as the life cycle assessment tool (ISO, 1997). LCA is incorporated in the ISO 14000 family of standards and is based on inventories of materials and quantification of adverse impacts. Such tools allow the use of a number of quantitative approaches, e.g. mass flow analysis (Brunner and Rechberger, 2004), and life cycle costing (Rebitzer and Hunkeler, 2003). Thereby, assessments can be made to identify how to develop more sustainable systems providing that the information is available. These assessments are difficult to perform in the developed world and there is, as yet, no consensus on a consistent methodology to measure these causes or effects. This is highlighted in the ongoing work of the life cycle impact assessment (LCIA) workgroup of the United Nations Environmental Programme (UNEP) global life cycle initiative (UNEP and SETAC, 2005). In the developing world there is often not enough quantitative information to follow these types of formalised methodologies (Brent, 2004).

In the developing country context, the availability of technology can also be seen to be a major limitation to introducing safe HCWM systems. Developing countries often do not have support systems to install, maintain, and operate developed world environmental technologies. The sustainable approach is to accommodate the use of more environmentally sound technologies (ESTs $)^{\frac{5}{}}$ in the design of the waste management system. These ESTs are safer than the technologies they replace and more suited to the economic, social, and environmental conditions of the developing country, which can be contextually unique (Tanner, 2005).

A checklist for the identification of ESTs is as follows (UN, 1992):

- Know-how in the organization adopting the EST;

- Procedures to use the EST in the organization; 
- Equipment and local technical support available;

- Organizational and managerial procedures with trained personnel; and

- Compatibility with nationally determined socio-economic, cultural, and environmental priorities.

In the absence of quantitative data on impacts and descriptions of systems capability to use ESTs in developing countries, a process is required by which the priorities can be set to establish safer systems.

1.3. Application of the AHP as decision support for the sustainable development of health care waste management (HCWM) systems

The complexities of health care waste management (HCWM) systems result from the many possible combinations of options, or alternatives, apart from singular technologies. However, when multi-criteria decision analysis (MCDA) models, such as the AHP, are used to consider waste management options, the models identified in the literature only take into account waste once generated (Morrissey and Browne, 2004). Waste prevention, waste minimisation, or product design for the environment, which would eliminate the production of materials that cannot be reused, recycled, or naturally biodegraded, are generally not considered (Morrissey and Browne, 2004). With respect to the sustainability of HCWM systems, the problems with accidental infection of health care personnel and patients have been recognized and safer technical alternatives are being researched (Dziekan et al., 2003) with the support of developing countries. In contrast to non-hazardous waste management approaches to sustainability, little can be achieved to prevent or minimise infectious waste, for which reuse, recycling, or natural biodegradation are not recommended due to safety and cost considerations (Rogers, 2004). In the developing country context, especially, the focus is currently on the management of generated waste in terms of

- Technical aspects, i.e. the most appropriate technologies (as discussed above) and/or procedures for health care facilities must be identified with respect to, for example, the 
availability, capacity, and maintainability (Rogers and Brent, in press and Rogers et al., 2001);

- Costs, i.e. costs must be minimised at national, district, and facility level (SA DoH, 2006 and Rogers et al., 2001); and

- Risks, i.e. health care facilities typically generate infectious (hazardous) waste with associated risks to human health (occupational and societal) and the natural environment, and these must be minimised (SA DEAT, 1998).

This paper concentrates on the infection risks related to the generated waste in HCWM systems, and specifically the waste generated at primary health care facilities ${ }^{\underline{6}}$ in rural areas of developing countries, in order to address the main research question: can the AHP be applied meaningfully in the context of sustainable development to establish HCWM systems that minimise infection risks in developing countries?

\subsection{Objectives of the research study}

The primary objectives of the research study were therefore:

- To propose a model, which integrates the AHP with other systems approaches in order to establish primary HCWM systems that minimise infection risks in developing countries; and

- To identify the key barriers to apply the AHP within such a model, which must be addressed when applying the model.

\section{Research methodology}

A case study research methodology was followed to evaluate the application of the AHP to HCWM systems. Case study methodology is preferred in examining contemporary events (Yin, 1989), and adding direct observations and systematic interviewing makes case studies more relevant than other strategies. However, a common concern about case studies is that they provide very little basis for scientific generalisation (Yin, 1989). The 
case studies consisted of two workshops in South Africa and Lesotho, with participants from the respective HCWM systems, where the AHP was applied. Therefore, the outcomes of this study are applicable in the Southern African context, but may not be correct for developing countries in other regions of the world.

The implications of this research approach were that four quality data tests, which have been noted as important (Reige, 2003), had to be verified. These tests, together with the associated techniques followed were (Reige, 2003 and Ramabitsa-Siimane, 2006):

- Construct validity: Interviews and observations made during site visits at primary health care clinics were used to clarify aspects of the respective HCWM systems, and the use of ESTs, for the workshop participants.

- External validation: The definition of the scope and boundary of the study was optimised during the study design such that the generalisation of HCWM systems in the Southern African context could be achieved.

- Reliability: Data were recorded in as concrete a manner as possible, by comparing the current situations with recognized best practices and interventions proven in the developing country context.

- Internal validity: Diagrams and illustrations prepared in the data-compiling phase were validated during the workshops.

\subsection{The representation of the AHP workshops of Southern African HCWM systems}

The two workshops stretched over one and two days, for South Africa and Lesotho, respectively. It has been stated that the size of an assessment panel depends on the objectives of the assessment, resources, and time available. As little as 10 participants are sufficient (Noble, 2004). This panel should comprise stakeholders, facilitators, and experts, although the level of expertise need not be the same as long as all participants are familiar with the problem (Saaty, 1980). Furthermore, to focus the AHP process it has been advised to engage a small group of participants (Noble, 2004 and Saaty, 1986). 
In the case of the South African and Lesotho workshops, 11 individuals participated over the full duration of the workshops that represent different operational levels in the respective HCWM systems:

- Strategic planning, e.g. occupational health, environment, and technical government officials and public-private specialists;

- Implementation planning, e.g. infection control, and technical government officials and public-private specialists; and

- Implementation, e.g. health inspectors, and waste management technical specialists.

The selected panels were representative in number but deficient in public-private significance. Less participants represented non-governmental organizations and the private sector involved with the HCWM systems of the countries. However, the main problem with the under serviced rural primary health care facilities lies with the national Departments of Health and it is the responsibility under the Health Acts in both countries that the respective departments are responsible to ensure minimum treatment standards are achieved in both public and private sectors (SA DoH, 2003 and L MHSW, 1970). Therefore, a larger representation of the public sector is appropriate for these cases.

\subsection{The AHP workshops}

\subsubsection{Presentations}

Both the participants and the facilitators of the workshop gave presentations. The facilitators used presentations to highlight and inform the panel of the research, its objectives and expected outcomes, i.e. to establish weighting values for waste management options at primary health care facilities in South Africa and Lesotho. The panel was also introduced to the AHP. Participants presented informally certain aspects of the respective HCWM systems in the separate session described below. 


\subsubsection{Open discussions}

Throughout the workshop, participants were advised to ask questions, which were answered by both the facilitators and the participants. This encouraged openness and transparency into the current situation of the HCWM systems. The questions, answers, and comments emanating from the different sessions of the workshop were recorded and incorporated into the results analyses.

\subsubsection{Consensus}

Definitions for the following issues were reached by consensus:

- Statement of the HCWM problems;

- Solutions to the identified problems;

- The objectives of the HCWM systems;

- The definition of HCW in the South African and Lesotho context; and

- The definition of a rural primary health care facility.

The nominal group technique was used in this part of the process (Dunham, 1998). This was in pursuit of reaching a cohesive understanding for the whole group. Although it has been argued that aggregating assessment data without examining individual differences may lead to a false sense of group consensus (Coxon, 1982), the aggregate of a group will provide results that are superior to those of an individual (Noble, 2004). Furthermore, there is a synergistic effect to aggregating individual judgments (Forman and Peniwati, 1998).

\subsubsection{AHP questionnaires}

The hierarchical trees of attributes and options for HCWM systems were constructed prior to the workshops (see Section 3 below) using national experts and survey data. The trees were presented on flip charts when doing the comparisons of the different attributes 
and options. Two session leaders and an assistant conducted this process, as has been suggested (Saaty, 1986). The panel of participants reached an agreement to alter the attributes and options to suit the South African and Lesotho context. A computer terminal was also set up to consolidate the results as they were generated. It also helped the facilitators to identify inconsistency in the individual set of comparisons. Members of the panel could then be urged to alter their weighting factors in the case of poor consistency ratios.

3. Integrating life cycle thinking to construct appropriate hierarchy trees

A model was developed for primary HCWM systems, termed WasteOpt, which is based on the combination of the life cycle management (LCM) approach (Hunkeler et al., 2004), and specifically the standardised life cycle assessment (LCA) (ISO, 1997) and life cycle costing (LCC) (Rebitzer and Hunkeler, 2003) tools, and the analytical hierarchy process (AHP). It therefore inherits the benefits of both processes (see Table 1). AHP is often applied as part of the LCA tool to evaluate environmental performances of system alternatives (Pineda-Henson et al., 2002).

\section{Table 1.}

The benefits of LCM and AHP

\begin{tabular}{|l|l|}
\hline Life cycle management & Analytical hierarchy process \\
\hline $\begin{array}{l}\text { 1. LCM includes procedures for all } \\
\text { inputs and outputs of a system, over } \\
\text { time and space, although social } \\
\text { aspects are limited at present }\end{array}$ & $\begin{array}{l}\text { 1. Does not insist on consensus but synthesizes } \\
\text { a representative outcome from diverse } \\
\text { judgements }\end{array}$ \\
$\begin{array}{l}\text { 2. Quantitative outcomes mitigate the } \\
\text { use of emotions in decision-making }\end{array}$ & $\begin{array}{l}\text { 2. Considers priorities of factors in a system and } \\
\text { enables the selection of the best alternative } \\
\text { based on goals }\end{array}$ \\
\hline
\end{tabular}




\begin{tabular}{|l|l|}
\hline Life cycle management & Analytical hierarchy process \\
\hline issues pertaining to a system & $\begin{array}{l}\text { method for establishing priorities } \\
\text { in solving problems }\end{array}$ \\
$\begin{array}{l}\text { 5. Reflects the natural tendency of the mind to } \\
\text { sort elements of a system into hierarchies while } \\
\text { also tracking the logical consistency of } \\
\text { judgements used for determining priorities }\end{array}$ \\
\hline
\end{tabular}

3.1. Waste life cycle management at primary health care facilities

A generalised life cycle system of health care waste has been introduced before in the context of primary health care facilities in rural areas in developing countries (Rogers et al., 2002). The life cycle system consists of four distinct main phases, each with a number of components, which are generic to any HCWM system (see Fig. 2). The reference of the life cycle system is a unit of waste generated at a typical primary health care facility. The assumptions concerning the spatial boundaries and inventories of the life cycle system are

- Health care waste is segregated from general (non-hazardous) waste.

- The life cycle commences when medical supplies have served their purpose or function and are therefore classified as waste. This corresponds to the "cradle". Health care waste ends its life cycle at final disposal ("grave"). The point of exposure (or contact) of individuals to the waste along the life cycle is another boundary of the system.

- The life cycle is described as having the four phases of generation, storage, treatment, and disposal (see Fig. 2).

- Each life cycle phase has components, e.g. transport to move the waste between generation, storage, treatment, and disposal areas (Prüss et al., 1999 and Carr, 2003). 
- Options for each component can be specified in nationally accepted standards. For example, specific standards are documented for South Africa (SABS, 1993). However, in many developing countries, these standards are not available, in which case available options can be identified and ranked by national technical experts.

- Decision-makers of waste management practices at district level, who have the ultimate responsibility according to the South African regulations (SA DoH, 2003), can identify alternative systems based on combinations of these options for each life cycle phase.

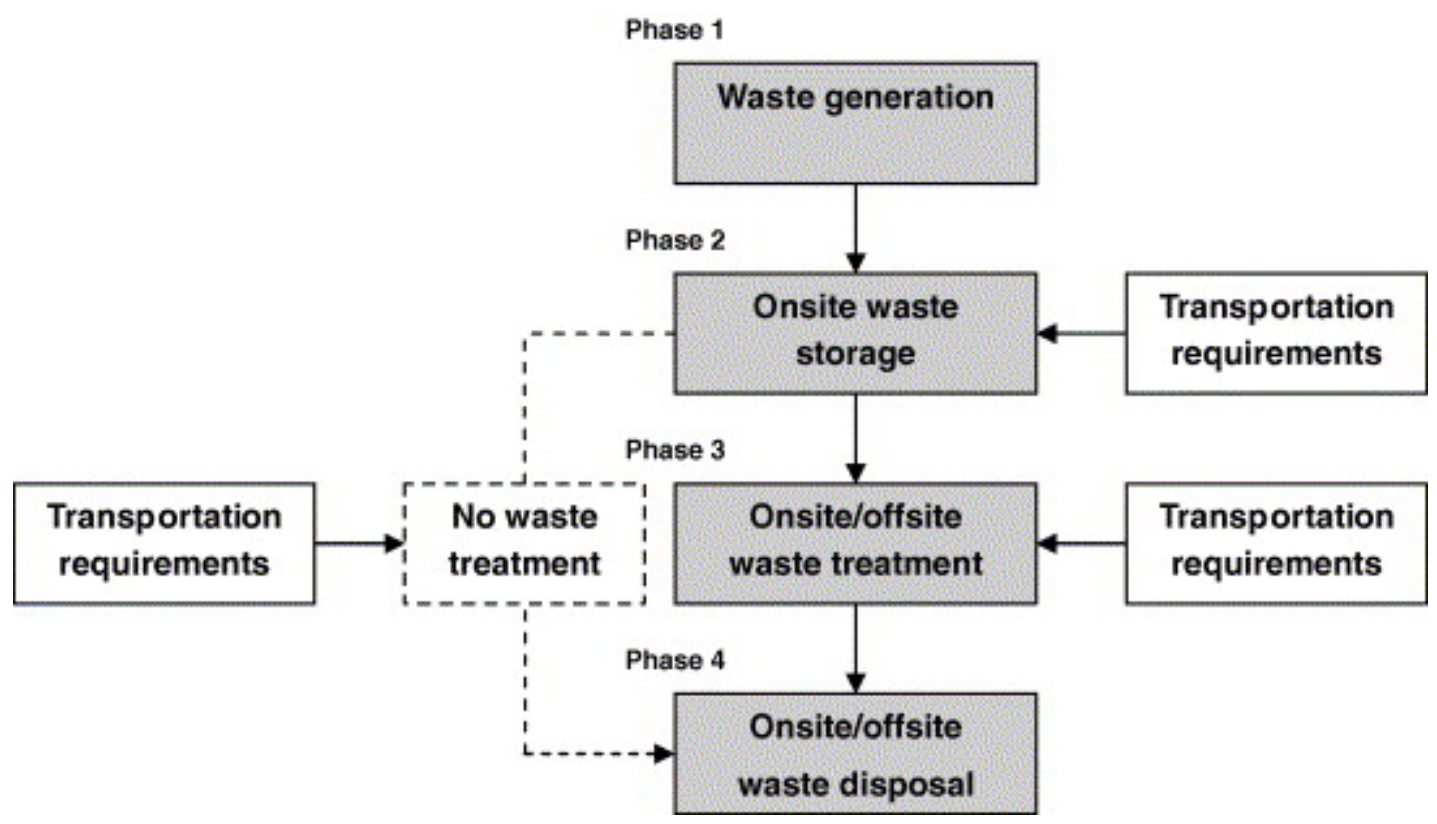

Fig. 2. The general life cycle of waste at primary health care facilities.

Databases have also been developed to provide examples of practical options for the life cycle phases (WHO, 2006). Although some of these options have been standardised (SABS, 1993), actual practices differ widely, which is due to choices based on the availability of working equipment, and financial resources, and training. The consequences of these choices are different levels of risk in terms of health impact due to various pathways to infection of health care workers, patients, and the public in general from the infectious waste, e.g. hepatitis B and HIV/AIDS via needle stick. 
The infection risks have been identified and assessed for certain options relating to treatment devices in terms of the procedure at the point where the health care waste is generated (Ekwueme et al., 2002). However, infection risks have not been assessed for the options for rural primary health care facilities for the other life cycle phase components. The approach followed here was to use expert opinion to place weights (in terms of infection risks, which are seen as the highest potential impact) on these options in the developing country context. These weights were used to compare alternatives for the four life cycle phases and to identify the alternative(s) with the minimum perceived infection risk. Ranking of the alternatives assists decision-makers to select the best available combination of options for the components of an entire HCWM system in a specific region.

\subsection{Correlating AHP and waste life cycle management terminology}

From the above it is necessary to correlate the different terminologies of the two decision support tools, if AHP is to be used to establish the (perceived) health risks associated with each option available for a life cycle phase component at rural primary health care facilities. The use of the AHP terms depends on the type of hierarchical tree and its application. Table 2 defines the terms of AHP when applied as part of the WasteOpt model.

\section{Table 2.}

Correlation of AHP and waste LCM terminology

\begin{tabular}{|l|l|l|}
\hline $\begin{array}{l}\text { Classified } \\
\text { hierarchy levels }\end{array}$ & $\begin{array}{l}\text { Conventional AHP } \\
\text { terminology }\end{array}$ & WasteOpt terminology \\
\hline Level 1 & Overall objective or focus & $\begin{array}{l}\text { Minimise infections risks for each } \\
\text { essential life cycle phase }\end{array}$ \\
\hline Level 2 & $\begin{array}{l}\text { Criteria, property, factor, } \\
\text { or influence }\end{array}$ & $\begin{array}{l}\text { Essential life cycle phase components, } \\
\text { and associated options }\end{array}$ \\
\hline Level 3 & $\begin{array}{l}\text { Alternatives, possibilities, } \\
\text { or outcomes }\end{array}$ & $\begin{array}{l}\text { Combination of life cycle phase } \\
\text { component options }\end{array}$ \\
\hline
\end{tabular}


Again, in the context of waste LCM, the overall objectives are to minimise the risk of infection at a clinic for a specific life cycle phase (see Fig. 2), and an alternative is therefore a combination of component options for each of the four main life cycle phases that can be chosen to minimise the overall risk of a HCWM system.

\subsection{Structuring of hierarchical trees of options for the life cycle phases}

The conventional AHP protocol (Saaty, 1980) requires that the elements be clustered into homogenous groups so they can meaningfully be compared with other elements in the level. It is also required that any element in one level must be capable of being related to some element in the next higher level. In a typical AHP the first level is the overall goal of the decision-maker. The second level consists of factors that contribute to the goal, while the third level denotes the alternatives available for application. Due to the fact that the factors in second level relate to a specific life cycle phase, individual hierarchies were constructed for each phase separately, as opposed to a single hierarchy for the entire system. Through the hierarchies, decision-makers are thereby enabled to select the preferred alternative for each life cycle phase. Options were identified for the life cycle components (of the phases) of waste systems at typical primary health care clinics in rural areas (see Fig. 2), and structured into the appropriate levels (see Table 2). These hierarchy trees are shown in Fig. 3, Fig. 4, Fig. 5, Fig. 6, Fig. 7 and Fig. 8 of Appendix A.

\section{Results of the workshops}

\subsection{Definitions of the hierarchical trees}

The definitions of each hierarchical tree (see Section 2.2.4) were arrived at by consensus of the participants of the workshops. While there was full consensus on the life cycle phases and the associated components, each country had its own set of technical options that were considered achievable. This reflects the level of technological development, i.e. South Africa has a more developed infrastructure. For this reason the South African group was in general agreement with the initially compiled life cycle phase components and associated options, whereas the group of participants of the Lesotho workshop made two major changes to the 
1. Options for the on-site disposal life cycle phase: The comparison for transport options was removed due to the argument that at the disposal site no transportation is practiced. The transport options for the treatment activity apply for the disposal activity as well, and a pit latrine was also considered to be a form of a controlled dump.

2. Options for central treatment activity: The incinerator technology comparisons were restricted to multi-chamber incinerator (MCI) and single-chamber incinerator (SCI) options. The consensus was reached by the group of experts and was justified by the fact that although the other techniques are in consideration for the country they have not been applied. Encapsulation treatment was also excluded because it was regarded as a stabilization method, which brought no change to the composition of the waste; it was therefore not considered a treatment method.

These changes are highlighted in Fig. 6 and Fig. 7 of Appendix A, respectively as the dashed outlined, non-shaded boxes.

\subsection{Priority weights of the life cycle phase components and options}

The AHP results of questionnaires for the two workshops are summarised in Table 4, Table 5, Table 6, Table 7, Table 8 and Table 9 of Appendix A. The tables highlight where individual participants were inconsistent within sets of comparisons. The original expectation was that a $10 \%$ consistency ratio $(\mathrm{CR})$ is required for valid results (Saaty, 1980). It has been suggested that consistency is particularly difficult when decisionmakers consider sustainable development aspects (Noble, 2004). However, for a 95\% confidence interval, the median $\mathrm{CR}$ can be up to $12 \%$ when dealing with a panel of multiple experts (Noble, 2004). Therefore, for these case studies, CRs of less than 12\% were considered as an adequate indication of consistency. The CRs of $12 \%$ and higher are bold in the tables of Appendix A.

The tables further show the average CRs for the individuals for all life cycle phase components, as well as the geometric mean, average, and standard deviations for the two groups for each set of comparisons. The calculations of the group average CRs only include those participants that were consistent in each set of comparisons, i.e. the CRs 
were less than 0.012 . The geometric mean weights for the different life cycle phase components and options, for the South African workshop, are summarised in the figures of Appendix A.

\section{Discussion}

5.1. The allocated time for the facilitation and coordination of the workshop process as a key issue in developing countries

The number of individual inconsistencies, when comparing the results of the two workshops in the tables of Appendix A, highlights the importance of the workshop process.

The length of time allocated to an AHP-dedicated workshop is of major importance. Because of the difficulty in keeping groups of people together, only one or two days for decision analysing are considered achievable. For example, in the Lesotho workshop additional people arrived on the second day and some left after the first day.

The one day that was allowed for the South African workshop, compared to the two days allocated to the Lesotho workshop, influenced the

- Time available for group discussions and subsequent consensus within sub-groups and the whole group in some cases; and

- The number of iterations; where inconsistencies were detected during the Lesotho workshop, subsequent iterations could be undertaken after further discussion, which has been shown to dramatically improve the CRs (Noble, 2004).

In another study where sustainable development aspects had to be weighted (Mulder and Brent, 2006), up to 5 days were required to reach good individual consistency within a panel of experts in a developing country context. This points to other limitations when applying the AHP. 


\subsection{The problem of diverse decision-makers in HCWM systems}

There were diverse participants in the two workshops, i.e. managers and implementers. The implementers at district level participated to acquire specific knowledge while the national managers argued at a higher level since they have more knowledge of the HCWM systems in general. The implementers were found to be "black or white" thinkers while the national experts perceived "grey" areas. The implementers subsequently formed sub-groups and could reach consensus amongst them, while the national planners differed in opinions and tended not to reach consensus in groups.

The ranking within the AHP by some national planners was taken as a separate judgement call for each comparison, instead of a judgement ranking within a set of comparisons. Therefore this level of participants tended to see discrete sets of options within an attribute option set. It was found to be very difficult to communicate this aspect of the AHP to the group at large. In these cases discussions between the workshop facilitators and individuals were required to inform participants of inconsistencies and how these come about, which, again, required additional time.

Some of the participants performed the ranking with pre-conceived ideas, which also negatively influenced consistencies within sets of comparisons. It is subsequently believed that direct weighing is easier and more relevant than pair-wise comparisons for these types of participants (Brent et al., 2005).

Some participants thought the conventional AHP scale of 1-9 was too small and did not allow them to allocate numbers properly. In contrast the scale confused other participants such that they mixed the left and the right hand sides of the scale when confronted by the comparison question, e.g. which option is more important, and by how much, to minimise infection risks. Furthermore, precision errors with the conventional AHP scale and eigenvector approach have been noted in literature (Laininen and Hämäläinen, 2002), and the precision level of each of the comparisons cannot be guaranteed.

For these reasons the spread of priority weights is rather large for some of the hierarchical tree attributes, which has been shown to be characteristic of the application 
of AHP to sustainable development (Noble, 2004). Considering the standard deviation and mean values in the tables of Appendix A, large spreads are more pronounced for the life cycle phase components than the associated specific options of components. This highlights the difficulty for the multiple decision-making panels to achieve consistent ranking of items on the upper levels of the hierarchical trees.

\subsection{Applying the priority weights to rank alternatives and assign risk factors}

The overall weights for alternatives, i.e. the combination of option choices for the essential life cycle phase components, were obtained with the conventional AHP by multiplication of the priority weight of each component option by the priority weight of the associated life cycle phase component. Table 9 provides, as an example, the waste generation life cycle phase using the South African workshop data. Determining priority from a safety perspective through the following equation has translated this ranking into impact indicators of the risk of each alternative for a life cycle phase:

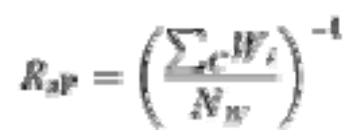

with $R_{\mathrm{aP}}=$ risk factor for an alternative in a life cycle phase, in relation to the risk factor of 1 for the alternative with the least risk for the phase; $W_{i}=$ overall weight for an option in a life cycle phase component $(C) ; N_{W}=$ normalisation value, i.e. the maximum combined weight from the choice of options for the components of a life cycle phase.

In Eq. (1) a risk factor is taken as the inverse for a normalised priority weighting. In other words, the alternative with the highest weight has the lowest risk factor and the alternative with the lowest weight the highest risk factor.

For the example of the waste generation life cycle phase, eight alternatives are possible. Using the overall weight values, the risk factors for the eight alternatives, using Eq. (1), are shown in Table 3. The risk factors for the other life cycle phases can be found elsewhere (Ramabitsa-Siimane, 2006). 


\section{Table 3.}

Assigning risk factors for the waste generation life cycle phase using the South African workshop results

\begin{tabular}{|l|l|}
\hline Options & Overall weights \\
\hline $\mathrm{EC}$ & 0.349 \\
\hline $\mathrm{NEC}$ & 0.045 \\
\hline $\mathrm{EL}$ & 0.200 \\
\hline $\mathrm{NEL}$ & 0.028 \\
\hline $\mathrm{DP}$ & 0.292 \\
\hline $\mathrm{NP}$ & 0.038 \\
\hline & \\
\hline Alternatives & Risk factors \\
\hline $\mathrm{EC}+\mathrm{EL}+\mathrm{DP}$ & 1.0 \\
\hline $\mathrm{nEC}+\mathrm{EL}+\mathrm{DP}$ & 1.6 \\
\hline $\mathrm{EC}+\mathrm{nEL}+\mathrm{DP}$ & 1.3 \\
\hline $\mathrm{nEC}+\mathrm{nEL}+\mathrm{DP}$ & 2.3 \\
\hline $\mathrm{EC}+\mathrm{EL}+\mathrm{nP}$ & 1.4 \\
\hline $\mathrm{nEC}+\mathrm{EL}+\mathrm{nP}$ & 3.0 \\
\hline $\mathrm{EC}+\mathrm{nEL}+\mathrm{nP}$ & 2.0 \\
\hline $\mathrm{nEC}+\mathrm{nEL}+\mathrm{nP}$ & 7.6 \\
\hline
\end{tabular}

An analysis of the risk factors in Table 3 shows a definite trend towards higher risk with less engineered technology and this is consistent with the approach used in the development of standards in the developed world (Grimmond et al., 2003). The largest risk reduction is associated with the introduction of an engineered safety box, e.g. from risk factor value of 7.6-2 in the last two alternatives. This risk reduction is consistent 
with the safety box policy of the World Health Organization (WHO) (Prüss et al., 1999). In the rural developing country situation, where training (DP) and working conditions (EL) are least controllable, the WHO intention is to ensure that at least there is a an engineered safety box in the generation life cycle phase.

The challenge of following this approach in the WasteOpt tool is the communication of the outcomes, i.e. the risk factors are not necessarily a true reflection of how much more risk is associated with one set of alternatives compared to another; it is only based on the combined perceptions of the selected panel.

\section{Conclusions and way forward}

A model is introduced that incorporates the standardised life cycle management (LCM) approach with the analytical hierarchy process (AHP), whereby health care waste management (HCWM) systems can be optimised in developing countries. The AHP was used to assign priorities to the alternatives in the life cycle of waste according to the infection risks associated with the life cycle component options that are available to decision-makers in the life cycle of waste. Thereby, alternatives, or combinations of options for essential components of a HCWM system were ranked according to the respective combined risks in relation to best practices for that developing country context. Also, allocations of resources for the implementation of these priorities can be assigned in short and medium terms based on availabilities of resources and largest benefit (Ramabitsa-Siimane, 2006). Therefore waste management systems can be optimized within the sustainability requirements of stakeholder acceptance, national safety requirements, and available system resources.

Two case studies were used to apply the AHP as part of the model. The case studies consisted of workshops that were held with stakeholders of the HCWM systems in South Africa and Lesotho. The case studies highlighted a number of barriers to the application of the AHP in developing countries, which must be addressed when applying the introduced WasteOpt tool: 
- The available time for a workshop: At least 2 days with two workshop facilitators and one assistant are required.

- Group size: A workshop should comprise of no more than 15 participants that represent the different stakeholder groups of the HCWM systems.

- Diversity of educational level and background of participants: Facilitators of workshops must take cognisance of the fact that the stakeholders of HCWM systems in developing countries represent different levels in public and private sectors, with varying educational backgrounds. Therefore separate discussions in subgroups may be necessary to reach consensus and thereby improve consistencies.

- AHP scale and set of comparisons: Much time is required to explain the AHP scale, the set of pair-wise comparisons, and the importance of consistency in order to achieve buyin to the process.

- Individual judgements: The problem of judgements, and possible outliers, has been noted (Laininen and Hämäläinen, 2002), which can be addressed with mathematical manipulation of the AHP matrices such as regression. At the very least it is important to report the intervals of priority weights (Mustajoki et al., 2005).

The choice of WasteOpt as a decision-making tool for developing country situations is based on its appropriateness to HCWM at all levels of administration and implementation in healthcare. Use of the hierarchy of waste management alone will not necessarily lead to economically and otherwise sustainable systems because it does not attempt to measure the impacts of the individual options available (White et al., 1995). The further development of the WasteOpt model would provide the benefits of ranking of options with little available information or data. 


\section{References}

Aguaron et al., 2003 J. Aguaron, M.T. Escobar and J.M. Moreno-Jimenez, Consistency stability intervals for a judgement in AHP decision support systems, European Journal of Operational Research 145 (2003), pp. 382-393.

Alidi, 1996 A.S. Alidi, Use of the analytical hierarchy process to measure the initial viability of industrial projects, International Journal of Project Management 14 (1996) (4), pp. 205-208.

Atkinson, 2000 G. Atkinson, Measuring corporate sustainability, Journal of Environmental Planning and Management 43 (2000) (2), pp. 235-252.

Azapagic and Perdan, 2000 A. Azapagic and S. Perdan, Indicator of sustainable development for industry: A general framework, Transactions on IchemE 78 (2000) (b), pp. $243-261$.

Bana et al., 1997 E. Bana, C.A. Costa, T.J. Stewart and J.C. Vansnick, Multicriteria decision analysis: Some thoughts based on the tutorial and discussion sessions of the ENIGMA meetings, European Journal of Operational Research 99 (1997), pp. 28-37.

Beynon et al., 2000 M. Beynon, B. Curry and P. Morgan, The Dempster-Shafer theory of evidence: An alternative approach to multicriteria decision modelling, Omega 28 (2000), pp. $37-50$.

Bititci et al., 2001 U.S. Bititci, P. Suwignjo and A.S. Carrie, Strategy management through quantitative modelling of performance measurement systems, International Journal of Production Economics 69 (2001), pp. 15-22.

Bodin and Gass, 2003 L. Bodin and S.I. Gass, On teaching the analytical hierarchy process, Computers and Operations Research 30 (2003) (10), pp. 1487-1498. 
Brent, 2004 A.C. Brent, A life cycle impact assessment procedure with resource groups as areas of protection, International Journal of Life Cycle Assessment 9 (2004) (3), pp. 172-179.

Brent et al., 2005 A.C. Brent, R. Heuberger and D. Manzini, Evaluating projects that are potentially eligible for clean development mechanism (CDM) funding in the South African context: A case study to establish weighting values for sustainable development criteria, Environmental and Development Economics 10 (2005) (5), pp. 631-649.

Briassoulis, $2001 \mathrm{H}$. Briassoulis, Sustainable development and its indicators: Through a (planner's) glass darkly, Journal of Environmental Planning and Management 44 (2001) (3), pp. 409-427.

Brunner and Rechberger, 2004 P.H. Brunner and H. Rechberger, Practical Handbook of Material Flow Analysis: Advanced Methods in Resource and Waste Management Series 1, CRC Press LLC, Florida (2004).

Bruntland, 1987 In: G. Bruntland, Editor, Our Common Future: The World Commission on Environment and Development, Oxford University Press, Oxford (1987).

Carmone et al., 1997 F.J. Carmone, A. Kara and S.H. Zanakis, A Monte Carlo investigation of incomplete pairwise comparison matrices in AHP, European Journal of Operational Research 102 (1997), pp. 538-553.

Carr, 2003 Carr, R. (Ed.), 2003. Decision-making guide for managing health-care waste from primary health-care centres. World Health Organization document, WHO/PHE/VAB/01.

Coxon, 1982 A.P. Coxon, The User's Guide to Multidimensional Scaling, Heinemann Educational Books, New Hampshire (1982).

Crabb, 2003 C. Crabb, Researchers argue that unsafe injections spread HIV more than unsafe sex, Bulletin of the World Health Organization 81 (2003) (4), p. 307. 
Dunham, 1998 Dunham, R.B., 1998. Nominal group technique: A users' guide. Organizational Behavior, University of Wisconsin. Available from:

$<$ http://instruction.bus.wisc.edu/obdemo/readings/ngt.html $>$, Accessed on 1 February 2006.

Dyer, 1990 J. Dyer, Remarks on the AHP, Management Science 36 (1990) (3), pp. 249258.

Dziekan et al., 2003 G. Dziekan, D. Chisholm, B. Johns, J. Rovira and Y.J.F. Hutin, The cost effectiveness of policies for the safe and appropriate use of injections in health care settings, Bulletin of the World Health Organization 81 (2003) (4), pp. 277-285.

Ekwueme et al., 2002 D.U. Ekwueme, B.G. Weniger and R.T. Chen, Model-based estimates of risks of disease transmission and economic costs of seven injection devices in sub-Saharan Africa, Bulletin of the World Health Organization 80 (2002) (11), pp. 859-870.

Forman and Peniwati, 1998 E. Forman and K. Peniwati, Aggregating individual judgments and priorities with the Analytical Hierarchy Process, European Journal of Operational Research 108 (1998), pp. 165-169.

Grimmond et al., 2003 T. Grimmond, T. Rings, C. Taylor, R. Creech, R. Kampen, W. Kable, P. Mead, P. Mackie and R. Pandur, Sharps injury reduction using Sharpssmart ${ }^{\text {TM}}$ : A reusable sharps management system, Journal of Hospital Infection 54 (2003), pp. 232238.

Hanley et al., 1997 N. Hanley, J. Shogren and B. White, Environmental Economics in Theory and Practice, Palgrave Macmillan, United Kingdom (1997).

Hokkanen and Salminen, 1997 J. Hokkanen and P. Salminen, Choosing a solid waste management system using multicriteria decision analysis, European Journal of Operational Research 98 (1997), pp. 19-36. 
Hughes, 1990 W.R. Hughes, A note on consistency in utility assessments, Decision Sciences 21 (1990) (4), pp. 882-887.

Hunkeler et al., 2004 Hunkeler, D., Saur, K., Rebitzer, G., Finkbeiner, M., Schmidt, W.P., Jensen, A.A., Stranddorf, H., Christiansen, K., 2004. Life-cycle management. Society of Environmental Toxicology and Chemistry (SETAC). Available from: $<$ http://www.setac.org>, Accessed on 1 February 2006.

ISO, 1997 International Organization for Standardisation (ISO), 1997. Environmental management - Life cycle assessment - Principles and framework. ISO 14040.

Johannessen et al., 2000 Johannessen, L.M., Dijkman, M., Bartone, C., Hanrahan, D., Boyer, M.G., Chandra, C., 2000. Health Care Waste Management guidance notes. Health, Nutrition and Population. The International Bank for Reconstruction and Development, World Bank, Washington.

Kamal, 2001 M.A.-S.A.-H. Kamal, Application of the AHP in project management, International Journal of Project Management 19 (2001), pp. 19-27.

Kane et al., 1999 A. Kane, J. Lloyd, M. Zaffran, L. Simonsen and J. Kane, Transmission of hepatitis B, hepatitis $C$, and human immunodeficiency viruses through unsafe injections in the developing world: Model based regional estimates, Bulletin of the World Health Organization 77 (1999) (10), pp. 801-807.

Kurttila et al., 2000 M. Kurttila, M. Pesonen, J. Kangas and M. Kajanus, Utilizing the analytic hierarchy process (AHP) in SWOT analysis: A hybrid method and its application to a forest-certification case, Forest Policy and Economics 1 (2000), pp. 41-52.

Labuschagne et al., 2005a C. Labuschagne, A.C. Brent and S.J. Claasen, Environmental and social impact considerations for sustainable project life cycle management in the process industry, Corporate Social Responsibility and Environmental Management 12 (2005) (1), pp. 38-54. 
Labuschagne et al., 2005b C. Labuschagne, A.C. Brent and R.P.G. van Erck, Assessing the sustainability performances of industries, Journal of Cleaner Production 13 (2005) (4), pp. 373-385.

Lai et al., 1999 V.S. Lai, R.P. Trueblood and B.K. Wong, Software selection: A case study of the application of the analytical hierarchy process to the selection of a multimedia authoring system, Information and Management 36 (1999) (4), pp. 221-232.

Laininen and Hämäläinen, 2002 P. Laininen and R.P. Hämäläinen, Analyzing AHPmatrices by regression, European Journal of Operational Research 148 (2002), pp. 514524.

L MHSW, 1970 Lesotho Ministry of Health and Social Welfare (L MHSW), 1970. Public Health Act. Lesotho Government, Maseru.

Madu, 1994 C.N. Madu, A quality confidence procedure for GDSS application in multicriteria decision making, IIE Transactions 26 (1994) (3), pp. 31-39.

Mendoza and Prabhu, 2000 G.A. Mendoza and R. Prabhu, Development of methodology for selecting criteria and indicators for sustainable forest management: A case study on participatory assessment, Environmental Management 26 (2000) (6), pp. 659-673.

Mendoza and Prabhu, 2003 G.A. Mendoza and R. Prabhu, Qualitative multi-criteria approaches to assessing indicators of sustainable forest resource management, Forest Ecology and Management 172 (2003), pp. 329-343.

Morrissey and Browne, 2004 A.J. Morrissey and J. Browne, Waste management models and their application to sustainable waste management, Waste Management 24 (2004), pp. $297-308$.

Mulder and Brent, 2006 J. Mulder and A.C. Brent, Selection of sustainable rural agriculture projects in South Africa: Case studies in the LandCare programme, Journal of Sustainable Agriculture 28 (2006) (2), pp. 55-84. 
Mustajoki et al., 2005 J. Mustajoki, R.P. Hämäläinen and A. Salo, Decision support by Interval SMART/SWING: Incorporating imprecision in the SMART and SWING methods, Decision Sciences 36 (2005) (2), pp. 317-339.

Noble, 2004 B.F. Noble, Strategic environmental assessment quality assurance: Evaluating and improving the consistency of judgments in assessment panels, Environmental Impact Assessment Review 24 (2004), pp. 3-25.

Pineda-Henson et al., 2002 R. Pineda-Henson, A.B. Culaba and G.A. Mendoza, Evaluating environmental performance of pulp and paper manufacturing using the analytical hierarchy process and life-cycle assessment, Journal of Industrial Ecology 6 (2002) (1), pp. 15-28.

Prüss et al., 1999 In: A. Prüss, E. Giroult and P. Rushbrook, Editors, Safe Management of Wastes from Health-care Activities, World Health Organization, Geneva (1999).

Qureshi et al., 1999 M.E. Qureshi, S.R. Harrison and M.K. Wegener, Validation of multicriteria analysis models, Agricultural Systems 62 (1999), pp. 105-116.

Ramabitsa-Siimane, 2006 Ramabitsa-Siimane, T.S.M., 2006. The identification of environmentally sound technologies for healthcare waste management in Lesotho. Masters thesis, Department of Engineering and Technology Management, University of Pretoria.

Rebitzer and Hunkeler, 2003 G. Rebitzer and D. Hunkeler, Life cycle costing in LCM: Ambitions, opportunities and limitations, International Journal of Life Cycle Assessment 8 (2003) (5), pp. 253-256.

Reige, 2003 A.M. Reige, Validity and reliability tests in case study research: A literature review with "hands-on" applications for each research phase, Qualitative Market Research: An International Journal 6 (2003) (2), pp. 75-86. 
Rennings and Wiggering, 1997 K. Rennings and H. Wiggering, Steps towards indicators of sustainable development: Linking economic and ecological concepts, Ecological Economics 20 (1997), pp. 25-36.

Robèrt et al., 2002 K.-H. Robèrt, B. Schmidt-Bleek, J. Aloisi de Larderel, G. Basile, J.L. Jansen, R. Kuehr, P. Price Thomas, M. Suzuki, P. Hawken and M. Wackernagel, Strategic sustainable development: Selection, design and synergies of applied tools, Journal of Cleaner Production 10 (2002), pp. 197-214.

Rogers, 2004 Rogers, D.E.C., 2004. The safe management and minimization of Health Care Waste. Department of Environmental Affairs and Development Planning, Western Cape Province, South Africa.

Rogers and Brent, in press Rogers, D.E.C., Brent, A.C. Small-scale medical waste incinerators: Experiences and trials in South Africa. Waste Management, in press.

Rogers et al., 2001 Rogers, D.E.C., Mvuma, G.G., Hietkamp, S., 2001. Emergency assessment and planning for primary health care waste management in Malawi. South African Council for Scientific and Industrial Research, Material Science and Manufacturing report HT282.

Rogers et al., 2002 D.E.C. Rogers, A.C. Brent and M.B. Rohwer, Life cycle check as decision support tool for medical waste management in underdeveloped areas of Africa, International Waste Management Biennial Congress \& Exhibition, Proceedings WasteCon (2002) (1), pp. 123-133 Durban, South Africa.

Saaty, 1980 T.L. Saaty, The Analytic Hierarchy Process, McGraw-Hill, New York (1980).

Saaty, 1986 T.L. Saaty, Decision-Making for Leaders, McGraw-Hill, New York (1986).

Saaty, 1990 T.L. Saaty, Multicriteria Decision Making: The Analytic Hierarchy Process, RWS Publications, Pittsburgh (1990). 
SABS, 1993 South African Bureau of Standards (SABS), 1993. Handling and disposal of waste materials within health care facilities. Standards South Africa document, SANS 10248/SABS 0248, Pretoria, South Africa.

SA DEAT, 1998 South African Department of Environmental Affairs and Tourism (SA DEAT), 1998. National Environmental Management Act. No. 107 of 1998, Pretoria, Available from: <http://www.environment.gov.za $>$, Accessed on 1 February 2006.

SA DoH, 1998 South African Department of Health (SA DoH), 1998. Classification of services at health care facilities. South African Government, Pretoria.

SA DoH, 2003 South African Department of Health (SA DoH), 2003. National Health Act. No. 61 of 2003, Pretoria, Available from: $<$ http://www.doh.gov.za/docs/legislationf.html>, Accessed on 1 February 2006.

SA DoH, 2006 South African Department of Health (SA DoH), 2006. National Health Information System. Available from: <http://www.doh.gov.za/nhis/>, Accessed on 1 February 2006.

Tanner, 2005 M. Tanner, Strengthening district health systems, Bulletin of the World Health Organization 83 (2005) (4), p. 403.

UN, 1992 United Nations (UN), 1992. Sustainable Development - Agenda 21. United Nations Conference on Environment and Development, Rio de Janerio, Brazil. Available from: $<$ http://www.un.org/esa/sustdev/documents/agenda21/index.htm $>$, Accessed on 1 February 2006.

UNCSD, 2001 United Nations Commission on Sustainable Development (UNCSD), 2001. Indicators of sustainable development: Guidelines and methodologies. Available from: <http://www.un.org/esa/sustdev/isd.htm>, Accessed on 1 February 2006.

UNDP, 2005 United Nations Development Programme (UNDP), 2005. Human Development Report. Available from: <http://hdr.undp.org/reports/global/2005/>, Accessed on 1 February 2006. 
UNEP and SETAC, 2005 United Nations Environmental Programme (UNEP) and the Society of Environmental Toxicology and Chemistry (SETAC), 2005. Global life cycle initiative. Available from: <http://www.unep.fr/pc/sustain/lcinitiative/home.htm>, Accessed on 1 February 2006.

White et al., 1995 Pr. White, M. Franke and P. Hindle, Integrated Solid Waste Management: A Life Cycle Inventory, Blackie Academic and Professional (1995).

WHO, 1999 World Health Organization (WHO), 1999. Aide Memoire for a national strategy for safe and appropriate use of injections. Department of Blood Safety and Clinical Technology, Geneva.

WHO, 2002 World Health Organization (WHO), 2002. First do no harm: introducing auto-disable syringes and ensuring injection safety in immunization systems of developing countries. Expanded Programme on Immunization and the Department of the Protection of the Human Environment, WHO/V\& B/02.26.

WHO, 2004 World Health Organization (WHO), 2004. The world health report 2004 changing history. Available from: < http://www.who.int/whr/previous/en/index.html $>$, Accessed on 1 February 2006.

WHO, 2005 World Health Organisation (WHO), 2005. The world health report 2005 make every mother and child count. Available from: <http://www.who.int/whr/2005/en/index.html>, Accessed on 1 February 2006.

WHO, 2006 World Health Organization (WHO), 2006. Health-care waste management. Available from: <http://www.healthcarewaste.org/>, Accessed on 1 February 2006.

Yin, 1989 R.K. Yin, Case Study Research Design and Methods (second ed.), Sage Publications, California (1989). 


\section{Appendices}

\section{Appendix A. Hierarchical trees for the life cycle phases and research results}

See Fig. 3, Fig. 4, Fig. 5, Fig. 6, Fig. 7 and Fig. 8 and Table 4, Table 5, Table 6, Table 7, Table 8 and Table 9.

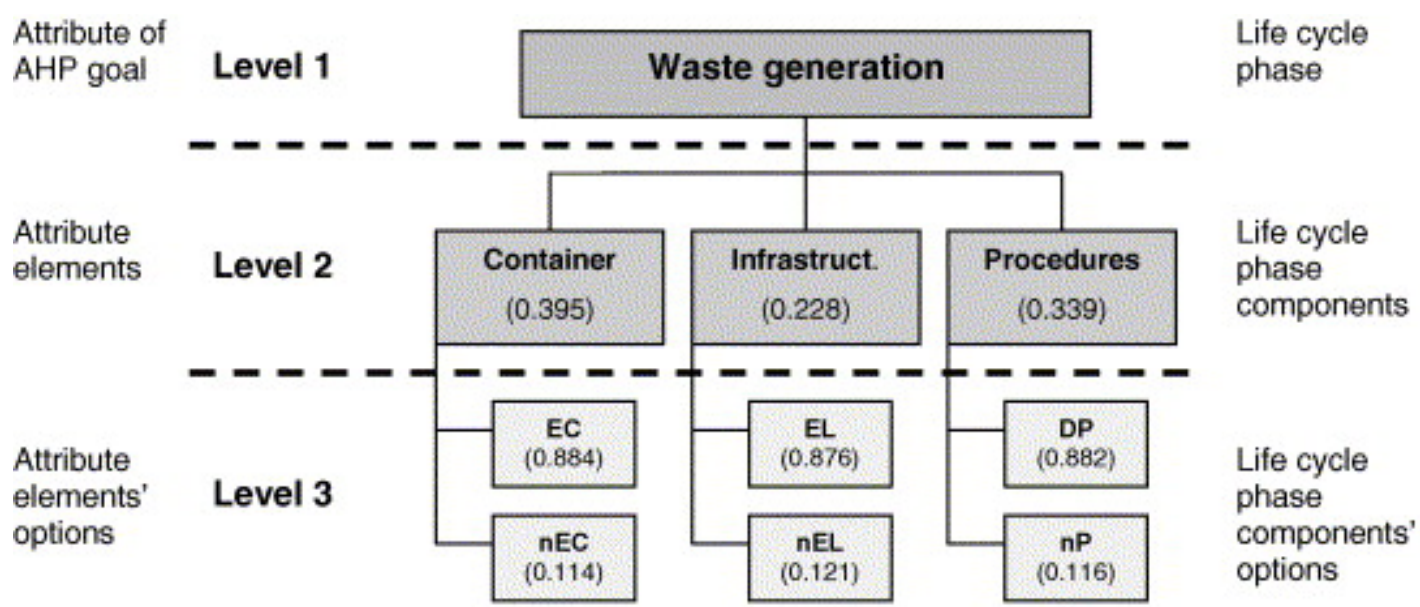

Alternatives are combinations of options, e.g. $\mathrm{nEC}+\mathrm{EL}+\mathrm{nP}$ 
Fig. 3. AHP options for the waste generation life cycle phase.

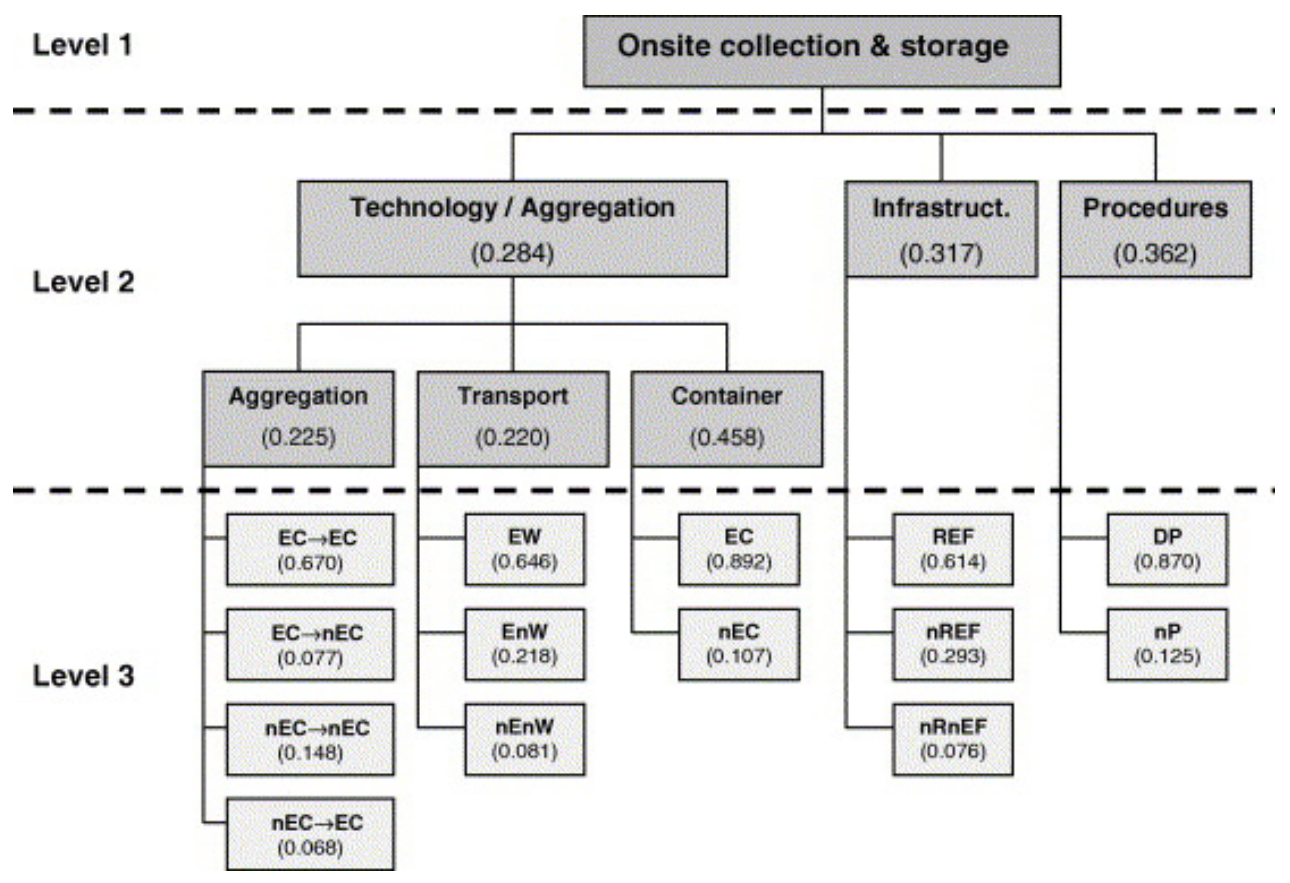

Fig. 4. AHP options for the onsite collection and storage life cycle phase.

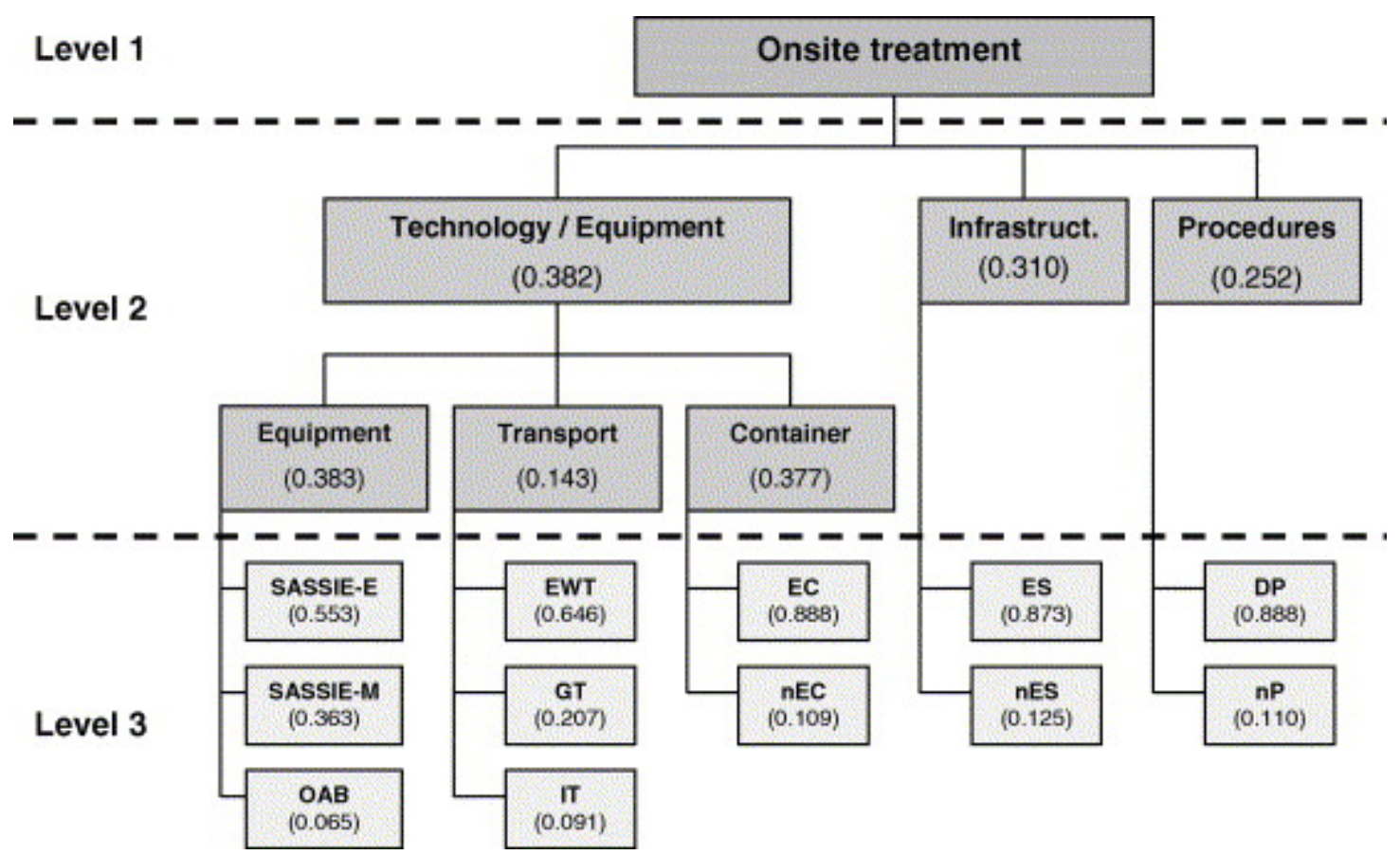


Fig. 5. AHP options for the onsite treatment waste life cycle phase.

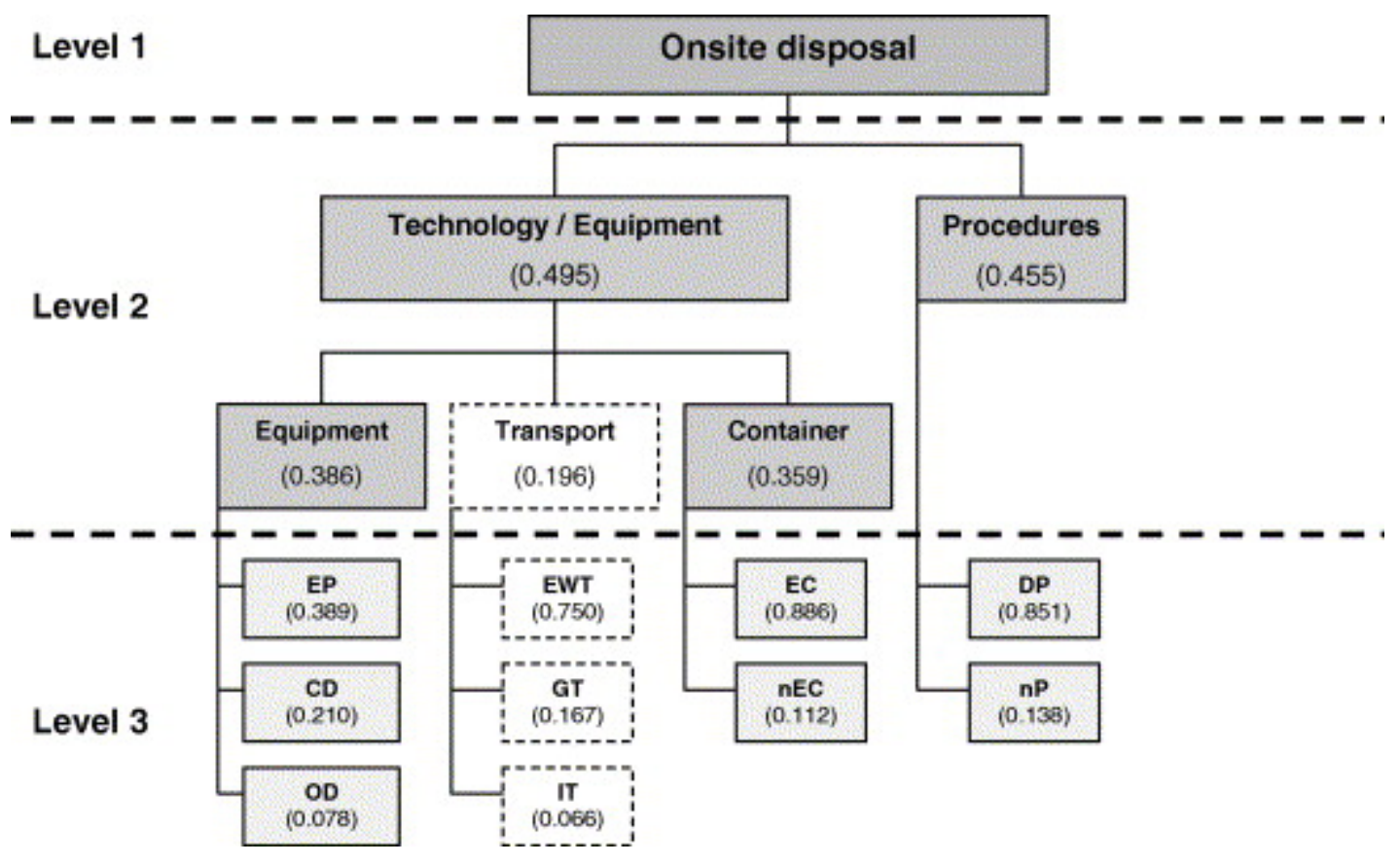

Fig. 6. AHP options for the onsite disposal waste life cycle phase.

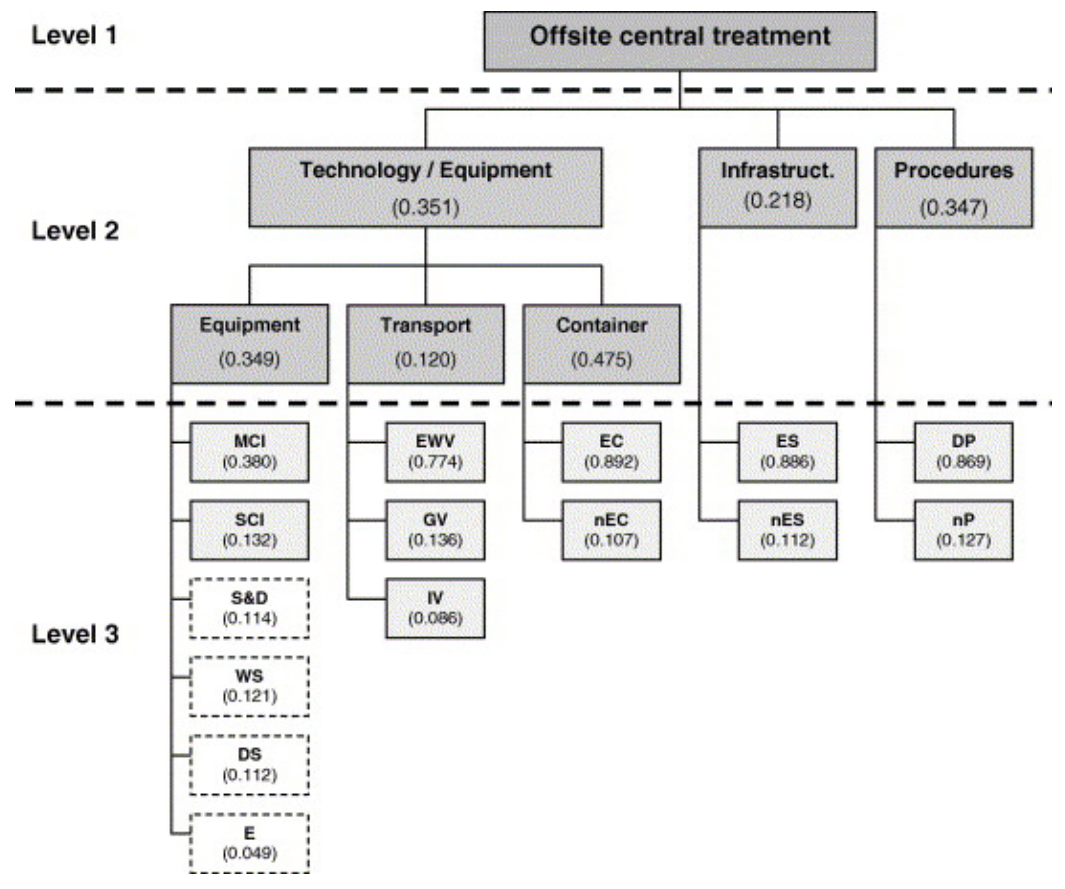


Fig. 7. AHP options for the offsite central treatment waste life cycle phase.

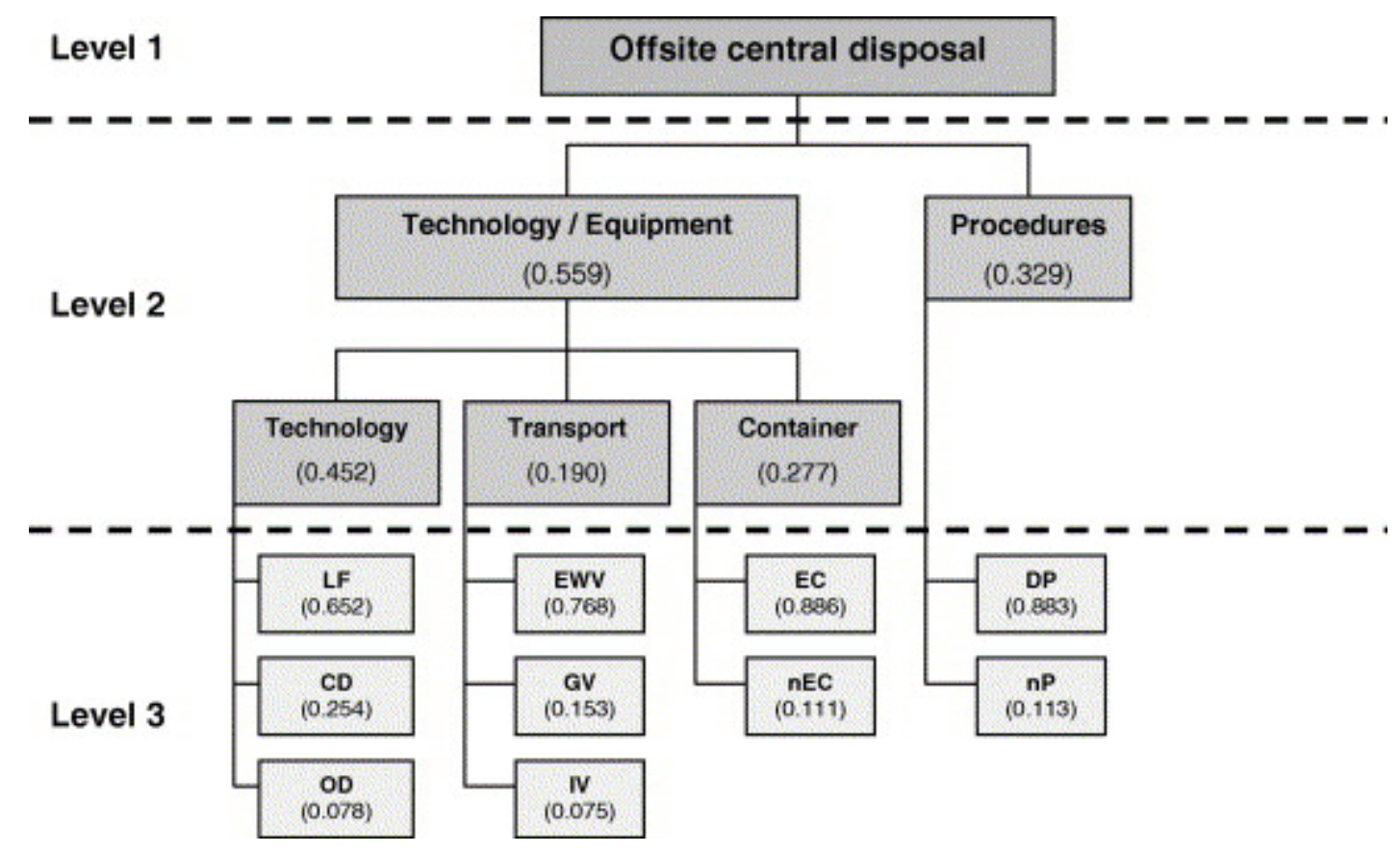

Fig. 8. AHP options for the offsite central disposal waste life cycle phase. 
Table 4.

AHP results for the waste generation life cycle phase

\begin{tabular}{|c|c|c|c|c|c|c|c|c|c|c|c|c|c|c|}
\hline \multirow{2}{*}{ Part. no. } & \multicolumn{4}{|c|}{ Level 2} & \multicolumn{3}{|c|}{ Level 3.1} & \multicolumn{3}{|c|}{ Level 3.2} & \multicolumn{3}{|c|}{ Level 3.3} & \multirow{2}{*}{ Avg. CR } \\
\hline & Cont. & Infra. & Proc. & CR & EC & nEC & $\mathbf{C R}$ & EL & nEL & $\mathbf{C R}$ & DP & $\mathbf{n P}$ & $\mathbf{C R}$ & \\
\hline \multicolumn{15}{|c|}{ South African workshop } \\
\hline 1 & 0.333 & 0.333 & 0.333 & 0.000 & 0.900 & 0.100 & 0.000 & 0.900 & 0.100 & 0.000 & 0.900 & 0.100 & 0.000 & 0.000 \\
\hline 2 & 0.460 & 0.221 & 0.319 & 0.117 & 0.900 & 0.100 & 0.000 & 0.889 & 0.111 & 0.000 & 0.889 & 0.111 & 0.000 & 0.029 \\
\hline 3 & 0.413 & 0.260 & 0.327 & 0.046 & 0.833 & 0.167 & 0.000 & 0.857 & 0.143 & 0.000 & 0.875 & 0.125 & 0.000 & 0.012 \\
\hline 4 & 0.400 & 0.400 & 0.200 & 0.000 & 0.875 & 0.125 & 0.000 & 0.875 & 0.125 & 0.000 & 0.833 & 0.167 & 0.000 & 0.000 \\
\hline 5 & 0.333 & 0.333 & 0.333 & 0.000 & 0.900 & 0.100 & 0.000 & 0.900 & 0.100 & 0.000 & 0.900 & 0.100 & 0.000 & 0.000 \\
\hline 6 & 0.333 & 0.333 & 0.333 & 0.000 & 0.900 & 0.100 & 0.000 & 0.900 & 0.100 & 0.000 & 0.900 & 0.100 & 0.000 & 0.000 \\
\hline 7 & 0.333 & 0.333 & 0.333 & 0.000 & 0.900 & 0.100 & 0.000 & 0.900 & 0.100 & 0.000 & 0.900 & 0.100 & 0.000 & 0.000 \\
\hline 8 & 0.333 & 0.333 & 0.333 & 0.000 & 0.900 & 0.100 & 0.000 & 0.900 & 0.100 & 0.000 & 0.900 & 0.100 & 0.000 & 0.000 \\
\hline 9 & 0.571 & 0.143 & 0.286 & 0.000 & 0.857 & 0.143 & 0.000 & 0.857 & 0.143 & 0.000 & 0.857 & 0.143 & 0.000 & 0.000 \\
\hline 10 & 0.455 & 0.091 & 0.455 & 0.056 & 0.875 & 0.125 & 0.000 & 0.833 & 0.167 & 0.000 & 0.875 & 0.125 & 0.000 & 0.014 \\
\hline 11 & 0.452 & 0.072 & 0.476 & 0.002 & 0.889 & 0.111 & 0.000 & 0.833 & 0.167 & 0.000 & 0.875 & 0.125 & 0.000 & 0.001 \\
\hline
\end{tabular}




\begin{tabular}{|c|c|c|c|c|c|c|c|c|c|c|c|c|c|c|}
\hline \multirow{2}{*}{ Part. no. } & \multicolumn{4}{|c|}{ Level 2} & \multicolumn{3}{|c|}{ Level 3.1} & \multicolumn{3}{|c|}{ Level 3.2} & \multicolumn{3}{|c|}{ Level 3.3} & \multirow{2}{*}{ Avg. CR } \\
\hline & Cont. & Infra. & Proc. & CR & EC & nEC & CR & EL & nEL & CR & DP & $\mathbf{n P}$ & CR & \\
\hline Mean & 0.395 & 0.228 & 0.331 & - & 0.884 & 0.114 & - & 0.876 & 0.121 & - & 0.882 & 0.116 & - & - \\
\hline Avg. & 0.401 & 0.259 & 0.339 & 0.020 & 0.884 & 0.116 & 0.000 & 0.877 & 0.123 & 0.000 & 0.882 & 0.118 & 0.000 & 0.005 \\
\hline Std. dev. & 0.078 & 0.112 & 0.074 & 0.038 & 0.022 & 0.022 & 0.000 & 0.027 & 0.027 & 0.000 & 0.022 & 0.022 & 0.000 & 0.010 \\
\hline \multicolumn{15}{|c|}{ Lesotho workshop } \\
\hline 1 & 0.413 & 0.260 & 0.327 & 0.046 & 0.833 & 0.167 & 0.000 & 0.875 & 0.125 & 0.000 & 0.875 & 0.125 & 0.000 & 0.012 \\
\hline 2 & 0.333 & 0.333 & 0.333 & 0.000 & 0.875 & 0.125 & 0.000 & 0.875 & 0.125 & 0.000 & 0.875 & 0.125 & 0.000 & 0.000 \\
\hline 3 & 0.333 & 0.333 & 0.333 & 0.000 & 0.900 & 0.100 & 0.000 & 0.900 & 0.100 & 0.000 & 0.900 & 0.100 & 0.000 & 0.000 \\
\hline 4 & 0.333 & 0.333 & 0.333 & 0.000 & 0.900 & 0.100 & 0.000 & 0.900 & 0.100 & 0.000 & 0.900 & 0.100 & 0.000 & 0.000 \\
\hline 5 & 0.333 & 0.333 & 0.333 & 0.000 & 0.900 & 0.100 & 0.000 & 0.900 & 0.100 & 0.000 & 0.900 & 0.100 & 0.000 & 0.000 \\
\hline 6 & 0.333 & 0.333 & 0.333 & 0.000 & 0.900 & 0.100 & 0.000 & 0.900 & 0.100 & 0.000 & 0.900 & 0.100 & 0.000 & 0.000 \\
\hline 7 & 0.333 & 0.333 & 0.333 & 0.000 & 0.900 & 0.100 & 0.000 & 0.900 & 0.100 & 0.000 & 0.900 & 0.100 & 0.000 & 0.000 \\
\hline 8 & 0.778 & 0.111 & 0.111 & 0.000 & 0.900 & 0.100 & 0.000 & 0.875 & 0.125 & 0.000 & 0.900 & 0.100 & 0.000 & 0.000 \\
\hline 9 & 0.279 & 0.072 & 0.649 & 0.056 & 0.900 & 0.100 & 0.000 & 0.900 & 0.100 & 0.000 & 0.900 & 0.100 & 0.000 & 0.014 \\
\hline 10 & 0.243 & 0.056 & 0.701 & 0.093 & 0.833 & 0.167 & 0.000 & 0.750 & 0.250 & 0.000 & 0.889 & 0.111 & 0.000 & 0.023 \\
\hline 11 & 0.455 & 0.091 & 0.455 & 0.000 & 0.857 & 0.143 & 0.000 & 0.800 & 0.200 & 0.000 & 0.857 & 0.143 & 0.000 & 0.000 \\
\hline
\end{tabular}




\begin{tabular}{|c|c|c|c|c|c|c|c|c|c|c|c|c|c|c|}
\hline \multirow{2}{*}{ Part. no. } & \multicolumn{4}{|c|}{ Level 2} & \multicolumn{3}{|c|}{ Level 3.1} & \multicolumn{3}{|c|}{ Level 3.2} & \multicolumn{3}{|c|}{ Level 3.3} & \multirow{2}{*}{ Avg. CF } \\
\hline & Cont. & Infra. & Proc. & CR & EC & nEC & CR & EL & nEL & CR & DP & $\mathbf{n P}$ & CR & \\
\hline Mean & 0.361 & 0.194 & 0.352 & - & 0.881 & 0.116 & - & 0.869 & 0.123 & - & 0.890 & 0.109 & - & - \\
\hline Avg. & 0.379 & 0.235 & 0.386 & 0.018 & 0.882 & 0.118 & 0.000 & 0.870 & 0.130 & 0.000 & 0.891 & 0.109 & 0.000 & 0.004 \\
\hline Std. dev. & 0.144 & 0.124 & 0.164 & 0.032 & 0.028 & 0.028 & 0.000 & 0.050 & 0.050 & 0.000 & 0.015 & 0.015 & 0.000 & 0.008 \\
\hline
\end{tabular}


Table 5.

AHP results for the waste collection and storage life cycle phase

\begin{tabular}{|c|c|c|c|c|c|c|c|c|c|c|c|c|c|c|c|c|c|c|c|c|c|c|c|c|c|c|c|c|}
\hline \multirow{2}{*}{$\begin{array}{l}\text { P } \\
\text { ar } \\
\text { t. } \\
\text { n } \\
\text { o. }\end{array}$} & \multicolumn{4}{|c|}{ Level 2.1} & \multicolumn{4}{|c|}{ Level 2.2} & \multicolumn{5}{|c|}{ Level 3.1} & \multicolumn{4}{|c|}{ Level 3.2} & \multicolumn{3}{|c|}{ Level 3.3} & \multicolumn{4}{|c|}{ Level 3.4} & \multicolumn{3}{|c|}{ Level 3.5} & \multirow{2}{*}{$\begin{array}{l}\text { A } \\
\text { vg } \\
\dot{C} \\
\text { C }\end{array}$} \\
\hline & $\begin{array}{l}\text { Tec } \\
\text { h./a } \\
\text { gg. }\end{array}$ & $\begin{array}{l}\text { In } \\
\text { fr } \\
\text { a. }\end{array}$ & $\begin{array}{l}\mathbf{P} \\
\text { ro } \\
\text { c. }\end{array}$ & $\begin{array}{l}\mathbf{C} \\
\mathbf{R}\end{array}$ & $\begin{array}{l}\text { A } \\
\text { gg } \\
\cdot\end{array}$ & $\begin{array}{l}\text { Tr } \\
\text { an } \\
\text {. }\end{array}$ & $\begin{array}{l}\text { C } \\
\text { on } \\
\text { t. }\end{array}$ & $\begin{array}{l}\mathbf{C} \\
\mathbf{R}\end{array}$ & $\begin{array}{l}\mathrm{EC} \\
>\mathbf{E} \\
\mathbf{C}\end{array}$ & $\begin{array}{l}\text { EC } \\
>\mathbf{n} \\
\text { EC }\end{array}$ & $\begin{array}{l}\mathbf{n E C} \\
>\mathbf{E} \\
\mathrm{C}\end{array}$ & $\begin{array}{l}\mathrm{nEC} \\
>\mathrm{nE} \\
\mathrm{C}\end{array}$ & $\begin{array}{l}\mathbf{C} \\
\mathbf{R}\end{array}$ & $\begin{array}{l}\mathbf{E} \\
\mathbf{W}\end{array}$ & $\begin{array}{l}\mathbf{n} \\
\mathbf{E} \\
\mathbf{W}\end{array}$ & $\begin{array}{l}\mathbf{n} \\
\mathbf{E} \\
\mathbf{n} \\
\mathbf{W}\end{array}$ & $\begin{array}{l}\mathbf{C} \\
\mathbf{R}\end{array}$ & $\begin{array}{l}\mathbf{E} \\
\mathbf{C}\end{array}$ & $\begin{array}{l}\mathbf{n} \\
\mathbf{E} \\
\mathbf{C}\end{array}$ & $\begin{array}{l}\mathbf{C} \\
\mathbf{R}\end{array}$ & $\begin{array}{l}\mathbf{R} \\
\mathbf{E} \\
\mathbf{F}\end{array}$ & $\begin{array}{l}\mathbf{n} \\
\mathbf{R} \\
\mathbf{E} \\
\mathbf{F}\end{array}$ & $\begin{array}{l}\mathbf{n} \\
\mathbf{R} \\
\mathbf{n} \\
\mathbf{E} \\
\mathbf{F}\end{array}$ & $\begin{array}{l}\mathbf{C} \\
\mathbf{R}\end{array}$ & $\begin{array}{l}\mathbf{D} \\
\mathbf{P}\end{array}$ & $\begin{array}{l}\mathbf{n} \\
\mathbf{P}\end{array}$ & $\begin{array}{l}\mathbf{C} \\
\mathbf{R}\end{array}$ & \\
\hline
\end{tabular}

South African workshop

\begin{tabular}{|c|c|c|c|c|c|c|c|c|c|c|c|c|c|c|c|c|c|c|c|c|c|c|c|c|c|c|c|c|}
\hline 1 & $\begin{array}{l}0.33 \\
3\end{array}$ & $\begin{array}{l}0 . \\
33 \\
3\end{array}$ & $\begin{array}{l}0 . \\
33 \\
3\end{array}$ & $\begin{array}{l}0 . \\
00 \\
0\end{array}$ & $\begin{array}{l}0 . \\
16 \\
7\end{array}$ & $\begin{array}{l}0 . \\
16 \\
7\end{array}$ & $\begin{array}{l}0 . \\
66 \\
7\end{array}$ & $\begin{array}{l}0 . \\
00 \\
0\end{array}$ & $\begin{array}{l}0.68 \\
3\end{array}$ & $\begin{array}{l}0.22 \\
8\end{array}$ & $\begin{array}{l}0.05 \\
2\end{array}$ & 0.037 & $\begin{array}{l}0 . \\
26 \\
2\end{array}$ & $\begin{array}{l}0 . \\
79 \\
6\end{array}$ & $\begin{array}{l}0 . \\
12 \\
5\end{array}$ & $\begin{array}{l}0 . \\
07 \\
9\end{array}$ & $\begin{array}{l}0 . \\
04 \\
6\end{array}$ & $\begin{array}{l}0 . \\
90 \\
0\end{array}$ & $\begin{array}{l}0 . \\
10 \\
0\end{array}$ & $\begin{array}{l}0 . \\
00 \\
0\end{array}$ & $\begin{array}{l}0 . \\
80 \\
2\end{array}$ & $\begin{array}{l}0 . \\
14 \\
2\end{array}$ & $\begin{array}{l}0 . \\
05 \\
6\end{array}$ & $\begin{array}{l}0 . \\
18 \\
7\end{array}$ & $\begin{array}{l}0 . \\
90 \\
0\end{array}$ & $\begin{array}{l}0 . \\
10 \\
0\end{array}$ & $\begin{array}{l}0 . \\
00 \\
0\end{array}$ & $\begin{array}{l}0 . \\
07 \\
1\end{array}$ \\
\hline 2 & $\begin{array}{l}0.54 \\
0\end{array}$ & $\begin{array}{l}0 . \\
16 \\
3\end{array}$ & $\begin{array}{l}0 . \\
29 \\
7\end{array}$ & $\begin{array}{l}\text { 0. } \\
31 \\
7\end{array}$ & $\begin{array}{l}0 . \\
47 \\
4\end{array}$ & $\begin{array}{l}0 . \\
14 \\
9\end{array}$ & $\begin{array}{l}0 . \\
37 \\
6\end{array}$ & $\begin{array}{l}0 . \\
04 \\
6\end{array}$ & $\begin{array}{l}0.74 \\
8\end{array}$ & $\begin{array}{l}0.09 \\
9\end{array}$ & $\begin{array}{l}0.08 \\
3\end{array}$ & 0.070 & $\begin{array}{l}0 . \\
02 \\
2\end{array}$ & $\begin{array}{l}0 . \\
73 \\
3\end{array}$ & $\begin{array}{l}0 . \\
22 \\
2\end{array}$ & $\begin{array}{l}0 . \\
04 \\
5\end{array}$ & $\begin{array}{l}\mathbf{0 .} \\
31 \\
7\end{array}$ & $\begin{array}{l}0 . \\
90 \\
0\end{array}$ & $\begin{array}{l}0 . \\
10 \\
0\end{array}$ & $\begin{array}{l}0 . \\
00 \\
0\end{array}$ & $\begin{array}{l}0 . \\
76 \\
6\end{array}$ & $\begin{array}{l}0 . \\
18 \\
3\end{array}$ & $\begin{array}{l}0 . \\
05 \\
1\end{array}$ & $\begin{array}{l}0 . \\
23 \\
2\end{array}$ & $\begin{array}{l}0 . \\
88 \\
9\end{array}$ & $\begin{array}{l}0 . \\
11 \\
1\end{array}$ & $\begin{array}{l}0 . \\
00 \\
0\end{array}$ & $\begin{array}{l}0 . \\
13 \\
3\end{array}$ \\
\hline 3 & $\begin{array}{l}0.46 \\
0\end{array}$ & $\begin{array}{l}0 . \\
22 \\
1\end{array}$ & $\begin{array}{l}0 . \\
31 \\
9\end{array}$ & $\begin{array}{l}0 . \\
11 \\
7\end{array}$ & $\begin{array}{l}0 . \\
26 \\
0\end{array}$ & $\begin{array}{l}0 . \\
21 \\
4\end{array}$ & $\begin{array}{l}0 . \\
52 \\
7\end{array}$ & $\begin{array}{l}\mathbf{0 .} \\
75 \\
1\end{array}$ & $\begin{array}{l}0.74 \\
4\end{array}$ & $\begin{array}{l}0.07 \\
0\end{array}$ & $\begin{array}{l}0.11 \\
7\end{array}$ & 0.070 & $\begin{array}{l}0 . \\
02 \\
2\end{array}$ & $\begin{array}{l}0 . \\
79 \\
6\end{array}$ & $\begin{array}{l}0 . \\
12 \\
5\end{array}$ & $\begin{array}{l}0 . \\
07 \\
9\end{array}$ & $\begin{array}{l}0 . \\
04 \\
6\end{array}$ & $\begin{array}{l}0 . \\
90 \\
0\end{array}$ & $\begin{array}{l}0 . \\
10 \\
0\end{array}$ & $\begin{array}{l}0 . \\
00 \\
0\end{array}$ & $\begin{array}{l}0 . \\
71 \\
1\end{array}$ & $\begin{array}{l}0 . \\
24 \\
3\end{array}$ & $\begin{array}{l}0 . \\
04 \\
6\end{array}$ & $\begin{array}{l}0 . \\
25 \\
4\end{array}$ & $\begin{array}{l}0 . \\
83 \\
3\end{array}$ & $\begin{array}{l}0 . \\
16 \\
7\end{array}$ & $\begin{array}{l}0 . \\
00 \\
0\end{array}$ & $\begin{array}{l}\text { 0. } \\
17 \\
0\end{array}$ \\
\hline 4 & $\begin{array}{l}0.58 \\
4\end{array}$ & $\begin{array}{l}0 . \\
23 \\
2\end{array}$ & $\begin{array}{l}0 . \\
18 \\
4\end{array}$ & $\begin{array}{l}0 . \\
18 \\
7\end{array}$ & $\begin{array}{l}0 . \\
15 \\
1\end{array}$ & $\begin{array}{l}0 . \\
21 \\
8\end{array}$ & $\begin{array}{l}0 . \\
63 \\
0\end{array}$ & $\begin{array}{l}0 . \\
09 \\
3\end{array}$ & $\begin{array}{l}0.63 \\
5\end{array}$ & $\begin{array}{l}0.07 \\
5\end{array}$ & $\begin{array}{l}0.21 \\
8\end{array}$ & 0.073 & $\begin{array}{l}0 . \\
00 \\
1\end{array}$ & $\begin{array}{l}0 . \\
42 \\
9\end{array}$ & $\begin{array}{l}0 . \\
42 \\
9\end{array}$ & $\begin{array}{l}0 . \\
14 \\
3\end{array}$ & $\begin{array}{l}0 . \\
00 \\
0\end{array}$ & $\begin{array}{l}0 . \\
87 \\
5\end{array}$ & $\begin{array}{l}0 . \\
12 \\
5\end{array}$ & $\begin{array}{l}0 . \\
00 \\
0\end{array}$ & $\begin{array}{l}0 . \\
69 \\
1\end{array}$ & $\begin{array}{l}0 . \\
21 \\
8\end{array}$ & $\begin{array}{l}0 . \\
09 \\
1\end{array}$ & $\begin{array}{l}0 . \\
04 \\
6\end{array}$ & $\begin{array}{l}0 . \\
83 \\
3\end{array}$ & $\begin{array}{l}0 . \\
16 \\
7\end{array}$ & $\begin{array}{l}0 . \\
00 \\
0\end{array}$ & $\begin{array}{l}0 . \\
04 \\
7\end{array}$ \\
\hline 5 & $\begin{array}{l}0.33 \\
3\end{array}$ & $\begin{array}{l}0 . \\
33 \\
3\end{array}$ & $\begin{array}{l}0 . \\
33 \\
3\end{array}$ & $\begin{array}{l}0 . \\
00 \\
0\end{array}$ & $\begin{array}{l}0 . \\
33 \\
3\end{array}$ & $\begin{array}{l}0 . \\
33 \\
3\end{array}$ & $\begin{array}{l}0 . \\
33 \\
3\end{array}$ & $\begin{array}{l}0 . \\
00 \\
0\end{array}$ & $\begin{array}{l}0.75 \\
0\end{array}$ & $\begin{array}{l}0.08 \\
3\end{array}$ & $\begin{array}{l}0.08 \\
3\end{array}$ & 0.083 & $\begin{array}{l}0 . \\
00 \\
0\end{array}$ & $\begin{array}{l}0 . \\
10 \\
0\end{array}$ & $\begin{array}{l}0 . \\
10 \\
0\end{array}$ & $\begin{array}{l}0 . \\
80 \\
0\end{array}$ & $\begin{array}{l}0 . \\
00 \\
0\end{array}$ & $\begin{array}{l}0 . \\
90 \\
0\end{array}$ & $\begin{array}{l}0 . \\
10 \\
0\end{array}$ & $\begin{array}{l}0 . \\
00 \\
0\end{array}$ & $\begin{array}{l}0 . \\
79 \\
7\end{array}$ & $\begin{array}{l}0 . \\
15 \\
1\end{array}$ & $\begin{array}{l}0 . \\
05 \\
2\end{array}$ & $\begin{array}{l}0 . \\
25 \\
4\end{array}$ & $\begin{array}{l}0 . \\
90 \\
0\end{array}$ & $\begin{array}{l}0 . \\
10 \\
0\end{array}$ & $\begin{array}{l}0 . \\
00 \\
0\end{array}$ & $\begin{array}{l}0 . \\
03 \\
6\end{array}$ \\
\hline
\end{tabular}




\begin{tabular}{|c|c|c|c|c|c|c|c|c|c|c|c|c|c|c|c|c|c|c|c|c|c|c|c|c|c|c|c|c|}
\hline \multirow{2}{*}{$\begin{array}{l}\text { P } \\
\text { ar } \\
\text { t. } \\
\text { n } \\
\text { o. }\end{array}$} & \multicolumn{4}{|c|}{ Level 2.1} & \multicolumn{4}{|c|}{ Level 2.2} & \multicolumn{5}{|c|}{ Level 3.1} & \multicolumn{4}{|c|}{ Level 3.2} & \multicolumn{3}{|c|}{ Level 3.3} & \multicolumn{4}{|c|}{ Level 3.4} & \multicolumn{3}{|c|}{ Level 3.5} & \multirow{2}{*}{$\begin{array}{l}\text { A } \\
\text { vg } \\
\text {. } \\
\text { C } \\
\text { R }\end{array}$} \\
\hline & $\begin{array}{l}\text { Tec } \\
\text { h./a } \\
\text { gg. }\end{array}$ & $\begin{array}{l}\text { In } \\
\text { fr } \\
\text { a. }\end{array}$ & $\begin{array}{l}\text { P } \\
\text { ro } \\
\text { C. }\end{array}$ & $\begin{array}{l}\mathbf{C} \\
\mathbf{R}\end{array}$ & $\begin{array}{l}\text { A } \\
\text { gg } \\
\cdot\end{array}$ & $\begin{array}{l}\text { Tr } \\
\text { an } \\
\cdot\end{array}$ & $\begin{array}{l}\text { C } \\
\text { on } \\
\text { t. }\end{array}$ & $\begin{array}{l}\mathbf{C} \\
\mathbf{R}\end{array}$ & $\begin{array}{l}\mathbf{E C} \\
>\mathbf{E} \\
\mathbf{C}\end{array}$ & $\begin{array}{l}\text { EC } \\
>\mathbf{n} \\
\text { EC }\end{array}$ & $\begin{array}{l}\text { nEC } \\
>E \\
C\end{array}$ & $\begin{array}{l}\mathbf{n E C} \\
>\mathbf{n E} \\
\mathbf{C}\end{array}$ & $\begin{array}{l}\mathbf{C} \\
\mathbf{R}\end{array}$ & $\begin{array}{l}\mathbf{E} \\
\mathbf{W}\end{array}$ & & & $\begin{array}{l}\mathbf{C} \\
\mathbf{R}\end{array}$ & $\begin{array}{l}\mathbf{E} \\
\mathbf{C}\end{array}$ & $\begin{array}{l}\mathbf{n} \\
\mathbf{E} \\
\mathbf{C}\end{array}$ & $\begin{array}{l}\mathbf{C} \\
\mathbf{R}\end{array}$ & $\begin{array}{l}\mathbf{R} \\
\mathbf{E} \\
\mathbf{F}\end{array}$ & $\begin{array}{l}\mathbf{n} \\
\mathbf{R} \\
\mathbf{E} \\
\mathbf{F}\end{array}$ & $\begin{array}{l}\mathbf{n} \\
\mathbf{R} \\
\mathbf{n} \\
\mathbf{E} \\
\mathbf{F}\end{array}$ & $\begin{array}{l}\mathbf{C} \\
\mathbf{R}\end{array}$ & $\begin{array}{l}\mathbf{D} \\
\mathbf{P}\end{array}$ & $\begin{array}{l}\mathbf{n} \\
\mathbf{P}\end{array}$ & $\begin{array}{l}\mathbf{C} \\
\mathbf{R}\end{array}$ & \\
\hline 6 & $\begin{array}{l}0.33 \\
3\end{array}$ & $\begin{array}{l}0 . \\
33 \\
3\end{array}$ & $\begin{array}{l}0 . \\
33 \\
3\end{array}$ & $\begin{array}{l}0 . \\
00 \\
0\end{array}$ & $\begin{array}{l}0 . \\
33 \\
3\end{array}$ & $\begin{array}{l}0 . \\
33 \\
3\end{array}$ & $\begin{array}{l}0 . \\
33 \\
3\end{array}$ & $\begin{array}{l}0 . \\
00 \\
0\end{array}$ & $\begin{array}{l}0.75 \\
0\end{array}$ & $\begin{array}{l}0.08 \\
3\end{array}$ & $\begin{array}{l}0.08 \\
3\end{array}$ & 0.083 & $\begin{array}{l}0 . \\
00 \\
0\end{array}$ & $\begin{array}{l}0 . \\
78 \\
3\end{array}$ & $\begin{array}{l}1 \\
4\end{array}$ & . & $\begin{array}{l}0 . \\
43 \\
1\end{array}$ & $\begin{array}{l}0 . \\
90 \\
0\end{array}$ & $\begin{array}{l}0 . \\
10 \\
0\end{array}$ & $\begin{array}{l}0 . \\
00 \\
0\end{array}$ & $\begin{array}{l}0 . \\
78 \\
7\end{array}$ & $\begin{array}{l}0 . \\
16 \\
7\end{array}$ & $\begin{array}{l}0 . \\
04 \\
6\end{array}$ & $\begin{array}{l}\mathbf{0} . \\
37 \\
6\end{array}$ & $\begin{array}{l}0 . \\
90 \\
0\end{array}$ & $\begin{array}{l}0 . \\
10 \\
0\end{array}$ & $\begin{array}{l}0 . \\
00 \\
0\end{array}$ & $\begin{array}{l}0 . \\
11 \\
5\end{array}$ \\
\hline 7 & $\begin{array}{l}0.33 \\
3\end{array}$ & $\begin{array}{l}0 . \\
33 \\
3\end{array}$ & $\begin{array}{l}0 . \\
33 \\
3\end{array}$ & $\begin{array}{l}0 . \\
00 \\
0\end{array}$ & $\begin{array}{l}0 . \\
33 \\
3\end{array}$ & $\begin{array}{l}0 . \\
33 \\
3\end{array}$ & $\begin{array}{l}0 . \\
33 \\
3\end{array}$ & $\begin{array}{l}0 . \\
00 \\
0\end{array}$ & $\begin{array}{l}0.69 \\
8\end{array}$ & $\begin{array}{l}0.07 \\
1\end{array}$ & $\begin{array}{l}0.19 \\
7\end{array}$ & 0.033 & $\begin{array}{l}0 . \\
31 \\
4\end{array}$ & $\begin{array}{l}0 . \\
81 \\
8\end{array}$ & $\begin{array}{l}0 \\
1\end{array}$ & ( & $\begin{array}{l}0 . \\
00 \\
0\end{array}$ & $\begin{array}{l}0 . \\
90 \\
0\end{array}$ & $\begin{array}{l}0 . \\
10 \\
0\end{array}$ & $\begin{array}{l}0 . \\
00 \\
0\end{array}$ & $\begin{array}{l}0 . \\
78 \\
3\end{array}$ & $\begin{array}{l}0 . \\
17 \\
4\end{array}$ & $\begin{array}{l}0 . \\
04 \\
3\end{array}$ & $\begin{array}{l}0 . \\
43 \\
1\end{array}$ & $\begin{array}{l}0 . \\
90 \\
0\end{array}$ & $\begin{array}{l}0 . \\
10 \\
0\end{array}$ & $\begin{array}{l}0 . \\
00 \\
0\end{array}$ & $\begin{array}{l}0 . \\
10 \\
6\end{array}$ \\
\hline 8 & $\begin{array}{l}0.33 \\
3\end{array}$ & $\begin{array}{l}0 . \\
33 \\
3\end{array}$ & $\begin{array}{l}0 . \\
33 \\
3\end{array}$ & $\begin{array}{l}0 . \\
00 \\
0\end{array}$ & $\begin{array}{l}0 . \\
33 \\
3\end{array}$ & $\begin{array}{l}0 . \\
33 \\
3\end{array}$ & $\begin{array}{l}0 . \\
33 \\
3\end{array}$ & $\begin{array}{l}0 . \\
00 \\
0\end{array}$ & $\begin{array}{l}0.53 \\
2\end{array}$ & $\begin{array}{l}0.05 \\
8\end{array}$ & $\begin{array}{l}0.35 \\
2\end{array}$ & 0.058 & $\begin{array}{l}0 . \\
01 \\
5\end{array}$ & $\begin{array}{l}0 . \\
65 \\
3\end{array}$ & 2 & 2 & $\begin{array}{l}0 . \\
06 \\
3\end{array}$ & $\begin{array}{l}0 . \\
88 \\
9\end{array}$ & $\begin{array}{l}0 . \\
11 \\
1\end{array}$ & $\begin{array}{l}0 . \\
00 \\
0\end{array}$ & $\begin{array}{l}0 . \\
65 \\
3\end{array}$ & $\begin{array}{l}0 . \\
28 \\
5\end{array}$ & $\begin{array}{l}0 . \\
06 \\
2\end{array}$ & $\begin{array}{l}0 . \\
06 \\
3\end{array}$ & $\begin{array}{l}0 . \\
88 \\
9\end{array}$ & $\begin{array}{l}0 . \\
11 \\
1\end{array}$ & $\begin{array}{l}0 . \\
00 \\
0\end{array}$ & $\begin{array}{l}0 . \\
02 \\
0\end{array}$ \\
\hline 9 & $\begin{array}{l}0.25 \\
0\end{array}$ & $\begin{array}{l}0 . \\
25 \\
0\end{array}$ & $\begin{array}{l}0 . \\
50 \\
0\end{array}$ & $\begin{array}{l}0 . \\
00 \\
0\end{array}$ & $\begin{array}{l}0 . \\
06 \\
7\end{array}$ & $\begin{array}{l}0 . \\
29 \\
3\end{array}$ & $\begin{array}{l}0 . \\
64 \\
1\end{array}$ & $\begin{array}{l}0 . \\
08 \\
6\end{array}$ & $\begin{array}{l}0.69 \\
5\end{array}$ & $\begin{array}{l}0.09 \\
9\end{array}$ & $\begin{array}{l}0.13 \\
1\end{array}$ & 0.075 & $\begin{array}{l}0 . \\
05 \\
7\end{array}$ & $\begin{array}{l}0 . \\
69 \\
1\end{array}$ & 8 & ( & $\begin{array}{l}0 . \\
04 \\
6\end{array}$ & $\begin{array}{l}0 . \\
87 \\
5\end{array}$ & $\begin{array}{l}0 . \\
12 \\
5\end{array}$ & $\begin{array}{l}0 . \\
00 \\
0\end{array}$ & $\begin{array}{l}0 . \\
63 \\
7\end{array}$ & $\begin{array}{l}0 . \\
25 \\
8\end{array}$ & $\begin{array}{l}0 . \\
10 \\
5\end{array}$ & $\begin{array}{l}0 . \\
03 \\
3\end{array}$ & $\begin{array}{l}0 . \\
80 \\
0\end{array}$ & $\begin{array}{l}0 . \\
20 \\
0\end{array}$ & $\begin{array}{l}0 . \\
00 \\
0\end{array}$ & $\begin{array}{l}0 . \\
03 \\
2\end{array}$ \\
\hline 10 & $\begin{array}{l}0.09 \\
1\end{array}$ & $\begin{array}{l}0 \\
45 \\
5\end{array}$ & $\begin{array}{l}0 . \\
45 \\
5\end{array}$ & $\begin{array}{l}0 . \\
00 \\
0\end{array}$ & $\begin{array}{l}0 . \\
07 \\
0\end{array}$ & $\begin{array}{l}0 . \\
17 \\
8\end{array}$ & $\begin{array}{l}0 . \\
75 \\
1\end{array}$ & $\begin{array}{l}0 . \\
02 \\
5\end{array}$ & $\begin{array}{l}0.55 \\
5\end{array}$ & $\begin{array}{l}0.05 \\
9\end{array}$ & $\begin{array}{l}0.34 \\
1\end{array}$ & 0.045 & $\begin{array}{l}0 . \\
10 \\
4\end{array}$ & $\begin{array}{l}0 . \\
65 \\
5\end{array}$ & 0 & 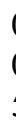 & $\begin{array}{l}0 . \\
06 \\
9\end{array}$ & $\begin{array}{l}0 . \\
87 \\
5\end{array}$ & $\begin{array}{l}0 . \\
12 \\
5\end{array}$ & $\begin{array}{l}0 . \\
00 \\
0\end{array}$ & $\begin{array}{l}0 . \\
65 \\
5\end{array}$ & $\begin{array}{l}0 . \\
29 \\
0\end{array}$ & $\begin{array}{l}0 . \\
05 \\
5\end{array}$ & $\begin{array}{l}0 . \\
06 \\
9\end{array}$ & $\begin{array}{l}0 . \\
83 \\
3\end{array}$ & $\begin{array}{l}0 . \\
16 \\
7\end{array}$ & $\begin{array}{l}0 . \\
00 \\
0\end{array}$ & $\begin{array}{l}0 . \\
03 \\
8\end{array}$ \\
\hline 11 & $\begin{array}{l}0.62 \\
2\end{array}$ & $\begin{array}{l}0 . \\
08 \\
1\end{array}$ & $\begin{array}{l}0 . \\
29 \\
7\end{array}$ & $\begin{array}{l}\mathbf{0 .} \\
37 \\
6\end{array}$ & $\begin{array}{l}0 . \\
48 \\
7\end{array}$ & $\begin{array}{l}0 . \\
07 \\
8\end{array}$ & $\begin{array}{l}0 . \\
43 \\
5\end{array}$ & $\begin{array}{l}0 . \\
01 \\
1\end{array}$ & $\begin{array}{l}0.71 \\
8\end{array}$ & $\begin{array}{l}0.13 \\
0\end{array}$ & $\begin{array}{l}0.11 \\
9\end{array}$ & 0.033 & $\begin{array}{l}0 . \\
15 \\
7\end{array}$ & $\begin{array}{l}0 . \\
46 \\
2\end{array}$ & 2 & , & $\begin{array}{l}0 . \\
00 \\
0\end{array}$ & $\begin{array}{l}0 . \\
90 \\
0\end{array}$ & $\begin{array}{l}0 . \\
10 \\
0\end{array}$ & $\begin{array}{l}0 . \\
00 \\
0\end{array}$ & $\begin{array}{l}0 . \\
46 \\
2\end{array}$ & $\begin{array}{l}0 . \\
46 \\
2\end{array}$ & $\begin{array}{l}0 . \\
07 \\
7\end{array}$ & $\begin{array}{l}0 . \\
00 \\
0\end{array}$ & $\begin{array}{l}0 . \\
90 \\
0\end{array}$ & $\begin{array}{l}0 . \\
10 \\
0\end{array}$ & $\begin{array}{l}0 . \\
00 \\
0\end{array}$ & $\begin{array}{l}0 . \\
07 \\
8\end{array}$ \\
\hline $\begin{array}{l}\text { M } \\
\text { ea }\end{array}$ & $\begin{array}{l}0.28 \\
4\end{array}$ & $\begin{array}{l}0 . \\
31\end{array}$ & $\begin{array}{l}0 . \\
36\end{array}$ & - & $\begin{array}{l}0 . \\
22\end{array}$ & $\begin{array}{l}0 . \\
22\end{array}$ & $\begin{array}{l}0 . \\
45\end{array}$ & - & $\begin{array}{l}0.67 \\
0\end{array}$ & $\begin{array}{l}0.07 \\
7\end{array}$ & $\begin{array}{l}0.14 \\
8\end{array}$ & 0.068 & - & $\begin{array}{l}0 . \\
64\end{array}$ & & & - & $\begin{array}{l}0 . \\
89\end{array}$ & $\begin{array}{l}0 . \\
10\end{array}$ & - & $\begin{array}{l}0 . \\
61\end{array}$ & $\begin{array}{l}0 . \\
29\end{array}$ & $\begin{array}{l}0 . \\
07\end{array}$ & - & $\begin{array}{l}0 . \\
87\end{array}$ & $\begin{array}{l}0 . \\
12\end{array}$ & - & - \\
\hline
\end{tabular}




\begin{tabular}{|c|c|c|c|c|c|c|c|c|c|c|c|c|c|c|c|c|c|c|c|c|c|c|c|c|c|c|c|c|c|c|}
\hline \multirow{2}{*}{$\begin{array}{l}\mathbf{P} \\
\text { ar } \\
\text { t. } \\
\text { n } \\
\text { o. }\end{array}$} & \multicolumn{4}{|c|}{ Level 2.1} & \multicolumn{4}{|c|}{ Level 2.2} & \multicolumn{5}{|c|}{ Level 3.1} & \multicolumn{4}{|c|}{ Level 3.2} & \multicolumn{4}{|c|}{ Level 3.3} & \multicolumn{5}{|c|}{ Level 3.4} & \multicolumn{3}{|c|}{ Level 3.5} & \multirow{2}{*}{$\begin{array}{l}\text { A } \\
\text { vg } \\
\dot{C} \\
\text { R }\end{array}$} \\
\hline & $\begin{array}{l}\text { Tec } \\
\text { h./a } \\
\text { gg. }\end{array}$ & $\begin{array}{l}\text { In } \\
\text { fr } \\
\text { a. }\end{array}$ & $\begin{array}{l}\mathbf{P} \\
\text { ro } \\
\text { c. }\end{array}$ & $\begin{array}{l}\mathbf{C} \\
\mathbf{R}\end{array}$ & $\begin{array}{l}\text { A } \\
\text { gg } \\
\cdot\end{array}$ & $\begin{array}{l}\text { Tr } \\
\text { an } \\
.\end{array}$ & $\begin{array}{l}\text { C } \\
\text { on } \\
\text { t. }\end{array}$ & $\begin{array}{l}\mathbf{C} \\
\mathbf{R}\end{array}$ & $\begin{array}{l}\mathbf{E C} \\
>\mathbf{E} \\
\mathbf{C}\end{array}$ & $\begin{array}{l}\text { EC } \\
>\text { n } \\
\text { EC }\end{array}$ & $\begin{array}{l}\mathrm{nEC} \\
>\mathrm{E} \\
\mathrm{C}\end{array}$ & $\begin{array}{l}\mathbf{n E C} \\
>\mathbf{n E} \\
\mathrm{C}\end{array}$ & $\begin{array}{l}\mathbf{C} \\
\mathbf{R}\end{array}$ & $\begin{array}{l}\mathbf{E} \\
\mathbf{W}\end{array}$ & $\begin{array}{l}\mathbf{n} \\
\mathbf{E} \\
\mathbf{W}\end{array}$ & $\begin{array}{l}\mathbf{n} \\
\mathbf{E} \\
\mathbf{n} \\
\mathbf{W}\end{array}$ & $\begin{array}{l}\mathbf{C} \\
\mathbf{R}\end{array}$ & E & & $\begin{array}{l}\mathrm{E} \\
\mathrm{E}\end{array}$ & $\begin{array}{l}\mathbf{C} \\
\mathbf{R}\end{array}$ & $\begin{array}{l}\mathbf{R} \\
\mathbf{E} \\
\mathbf{F}\end{array}$ & $\begin{array}{l}\mathbf{n} \\
\mathbf{R} \\
\mathbf{E} \\
\mathbf{F}\end{array}$ & $\begin{array}{l}\mathbf{E} \\
\mathbf{F}\end{array}$ & & $\begin{array}{l}\text { C } \\
\text { R }\end{array}$ & $\begin{array}{l}\mathbf{D} \\
\mathbf{P}\end{array}$ & $\begin{array}{l}\mathbf{n} \\
\mathbf{P}\end{array}$ & $\begin{array}{l}\mathbf{C} \\
\mathbf{R}\end{array}$ & \\
\hline $\mathrm{n}$ & & 7 & 2 & & 5 & 0 & 8 & & & & & & & 6 & 8 & 1 & & 2 & & 7 & & 4 & 3 & 6 & & & 0 & 5 & & \\
\hline $\begin{array}{l}\text { A } \\
\text { vg } \\
.\end{array}$ & $\begin{array}{l}0.30 \\
8\end{array}$ & $\begin{array}{l}0 . \\
32 \\
4\end{array}$ & $\begin{array}{l}0 . \\
36 \\
7\end{array}$ & $\begin{array}{l}0 . \\
01 \\
5\end{array}$ & $\begin{array}{l}0 . \\
27 \\
5\end{array}$ & $\begin{array}{l}0 . \\
24 \\
2\end{array}$ & $\begin{array}{l}0 . \\
48 \\
3\end{array}$ & $\begin{array}{l}0 . \\
02 \\
6\end{array}$ & $\begin{array}{l}0.67 \\
6\end{array}$ & $\begin{array}{l}0.07 \\
8\end{array}$ & $\begin{array}{l}0.17 \\
6\end{array}$ & 0.070 & $\begin{array}{l}0 . \\
02 \\
8\end{array}$ & $\begin{array}{l}0 . \\
66 \\
3\end{array}$ & $\begin{array}{l}0 . \\
25 \\
3\end{array}$ & $\begin{array}{l}0 . \\
08 \\
5\end{array}$ & $\begin{array}{l}0 . \\
03 \\
4\end{array}$ & $\begin{array}{l}8 \\
2\end{array}$ & & $\begin{array}{l}0 . \\
10 \\
3\end{array}$ & $\begin{array}{l}0 . \\
00 \\
0\end{array}$ & $\begin{array}{l}0 . \\
62 \\
0\end{array}$ & $\begin{array}{l}0 . \\
30 \\
3\end{array}$ & $\begin{array}{l}0 \\
8\end{array}$ & & $\begin{array}{l}0 . \\
04 \\
2\end{array}$ & $\begin{array}{l}0 . \\
87 \\
1\end{array}$ & $\begin{array}{l}0 . \\
12 \\
9\end{array}$ & $\begin{array}{l}0 . \\
00 \\
0\end{array}$ & $\begin{array}{l}0 . \\
07 \\
7\end{array}$ \\
\hline $\begin{array}{l}\text { St } \\
\text { d. } \\
\text { de } \\
\text { v. }\end{array}$ & $\begin{array}{l}0.10 \\
5\end{array}$ & $\begin{array}{l}0 . \\
06 \\
9\end{array}$ & $\begin{array}{l}0 . \\
06 \\
9\end{array}$ & $\begin{array}{l}0 . \\
04 \\
1\end{array}$ & $\begin{array}{l}0 . \\
15 \\
3\end{array}$ & $\begin{array}{l}0 . \\
09 \\
5\end{array}$ & $\begin{array}{l}0 . \\
16 \\
9\end{array}$ & $\begin{array}{l}0 . \\
03 \\
7\end{array}$ & $\begin{array}{l}0.09 \\
1\end{array}$ & $\begin{array}{l}0.01 \\
6\end{array}$ & $\begin{array}{l}0.11 \\
4\end{array}$ & 0.013 & $\begin{array}{l}0 . \\
03 \\
6\end{array}$ & $\begin{array}{l}0 . \\
14 \\
9\end{array}$ & $\begin{array}{l}0 . \\
14 \\
0\end{array}$ & $\begin{array}{l}0 . \\
02 \\
7\end{array}$ & $\begin{array}{l}0 . \\
02 \\
9\end{array}$ & $\begin{array}{l}2 \\
0\end{array}$ & & 21 & $\begin{array}{l}0 . \\
00 \\
0\end{array}$ & $\begin{array}{l}0 . \\
09 \\
0\end{array}$ & $\begin{array}{l}0 . \\
09 \\
4\end{array}$ & $\begin{array}{l}0 \\
1\end{array}$ & & $\begin{array}{l}0 . \\
02 \\
8\end{array}$ & $\begin{array}{l}0 . \\
21 \\
3\end{array}$ & $\begin{array}{l}0 . \\
21 \\
3\end{array}$ & $\begin{array}{l}0 . \\
00 \\
0\end{array}$ & $\begin{array}{l}0 . \\
05 \\
0\end{array}$ \\
\hline \multicolumn{31}{|c|}{ Lesotho workshop } \\
\hline 1 & $\begin{array}{l}0.06 \\
9\end{array}$ & $\begin{array}{l}0 . \\
24 \\
4\end{array}$ & $\begin{array}{l}0 . \\
68 \\
7\end{array}$ & $\begin{array}{l}0 . \\
10 \\
7\end{array}$ & $\begin{array}{l}0 . \\
05 \\
9\end{array}$ & $\begin{array}{l}0 . \\
24 \\
0\end{array}$ & $\begin{array}{l}0 . \\
70 \\
1\end{array}$ & $\begin{array}{l}0 . \\
25 \\
4\end{array}$ & $\begin{array}{l}0.29 \\
3\end{array}$ & $\begin{array}{l}0.29 \\
3\end{array}$ & $\begin{array}{l}0.20 \\
7\end{array}$ & 0.207 & $\begin{array}{l}0 . \\
04 \\
5\end{array}$ & $\begin{array}{l}0 . \\
73 \\
1\end{array}$ & $\begin{array}{l}0 . \\
18 \\
8\end{array}$ & $\begin{array}{l}0 . \\
08 \\
1\end{array}$ & $\begin{array}{l}0 . \\
0 \\
6\end{array}$ & 5 & & $\begin{array}{l}0 . \\
12\end{array}$ & $\begin{array}{l}0 . \\
00 \\
0\end{array}$ & $\begin{array}{l}0 . \\
73 \\
1\end{array}$ & $\begin{array}{l}0 . \\
18 \\
8\end{array}$ & 1 & & $\begin{array}{l}0 . \\
05 \\
6\end{array}$ & $\begin{array}{l}0 . \\
88 \\
9\end{array}$ & $\begin{array}{l}0 . \\
11 \\
1\end{array}$ & $\begin{array}{l}0 . \\
00 \\
0\end{array}$ & $\begin{array}{l}0 . \\
07 \\
4\end{array}$ \\
\hline 2 & $\begin{array}{l}0.06 \\
7\end{array}$ & $\begin{array}{l}0 . \\
46 \\
7\end{array}$ & $\begin{array}{l}0 . \\
46 \\
7\end{array}$ & $\begin{array}{l}0 . \\
00 \\
0\end{array}$ & $\begin{array}{l}0 . \\
06 \\
7\end{array}$ & $\begin{array}{l}0 . \\
46 \\
7\end{array}$ & $\begin{array}{l}0 . \\
46 \\
7\end{array}$ & $\begin{array}{l}0 . \\
00 \\
0\end{array}$ & $\begin{array}{l}0.37 \\
7\end{array}$ & $\begin{array}{l}0.07 \\
2\end{array}$ & $\begin{array}{l}0.49 \\
6\end{array}$ & 0.055 & $\begin{array}{l}0 . \\
05 \\
7\end{array}$ & $\begin{array}{l}0 . \\
73 \\
5\end{array}$ & $\begin{array}{l}0 . \\
20 \\
7\end{array}$ & $\begin{array}{l}0 . \\
05 \\
8\end{array}$ & $\begin{array}{l}0 . \\
10 \\
0\end{array}$ & 0 & & $\begin{array}{l}0 . \\
0\end{array}$ & $\begin{array}{l}0 . \\
00 \\
0\end{array}$ & $\begin{array}{l}0 . \\
77 \\
3\end{array}$ & $\begin{array}{l}0 . \\
13 \\
9\end{array}$ & 8 & & $\begin{array}{l}0 . \\
04 \\
6\end{array}$ & $\begin{array}{l}0 . \\
88 \\
9\end{array}$ & $\begin{array}{l}0 . \\
11 \\
1\end{array}$ & $\begin{array}{l}0 . \\
00 \\
0\end{array}$ & $\begin{array}{l}0 . \\
02 \\
9\end{array}$ \\
\hline 3 & $\begin{array}{l}0.06 \\
3\end{array}$ & $\begin{array}{l}0 . \\
45 \\
8\end{array}$ & $\begin{array}{l}0 . \\
47 \\
9\end{array}$ & $\begin{array}{l}0 . \\
00 \\
2\end{array}$ & $\begin{array}{l}0 . \\
81 \\
8\end{array}$ & $\begin{array}{l}0 . \\
09 \\
1\end{array}$ & $\begin{array}{l}0 . \\
09 \\
1\end{array}$ & $\begin{array}{l}0 . \\
00 \\
0\end{array}$ & $\begin{array}{l}0.37 \\
7\end{array}$ & $\begin{array}{l}0.07 \\
3\end{array}$ & $\begin{array}{l}0.49 \\
6\end{array}$ & 0.055 & $\begin{array}{l}0 . \\
05 \\
7\end{array}$ & $\begin{array}{l}0 . \\
74 \\
3\end{array}$ & $\begin{array}{l}0 . \\
19 \\
4\end{array}$ & $\begin{array}{l}0 . \\
06 \\
3\end{array}$ & $\begin{array}{l}0 \\
1\end{array}$ & 0 & & 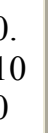 & $\begin{array}{l}0 . \\
00 \\
0\end{array}$ & $\begin{array}{l}0 . \\
81 \\
8\end{array}$ & $\begin{array}{l}0 . \\
09 \\
1\end{array}$ & 1 & & $\begin{array}{l}0 . \\
00 \\
0\end{array}$ & $\begin{array}{l}0 . \\
90 \\
0\end{array}$ & $\begin{array}{l}0 . \\
10 \\
0\end{array}$ & $\begin{array}{l}0 . \\
00 \\
0\end{array}$ & $\begin{array}{l}0 . \\
01 \\
7\end{array}$ \\
\hline 4 & 0.81 & 0. & 0 & 0 & 0 & 0 & 0. & 0 . & 0.20 & 0.20 & 0.29 & 0.293 & 0. & 0. & 0. & 0. & 0. & 0 & & 0. & 0. & 0. & 0 . & 0 & & 0. & 0 & 0. & 0. & 0. \\
\hline
\end{tabular}




\begin{tabular}{|c|c|c|c|c|c|c|c|c|c|c|c|c|c|c|c|c|c|c|c|c|c|c|c|c|c|c|c|c|c|c|}
\hline \multirow{3}{*}{$\begin{array}{l}\text { P } \\
\text { ar } \\
\text { t. } \\
\text { n } \\
\text { o. }\end{array}$} & \multicolumn{4}{|c|}{ Level 2.1} & \multicolumn{5}{|c|}{ Level 2.2} & \multicolumn{5}{|c|}{ Level 3.1} & \multicolumn{4}{|c|}{ Level 3.2} & \multicolumn{3}{|c|}{ Level 3.3} & \multicolumn{5}{|c|}{ Level 3.4} & \multicolumn{3}{|c|}{ Level 3.5} & \multirow{2}{*}{$\begin{array}{l}\text { A } \\
\text { vg } \\
\text {. } \\
\text { C } \\
\text { R }\end{array}$} \\
\hline & $\begin{array}{l}\text { Tec } \\
\text { h./a } \\
\text { gg. }\end{array}$ & $\begin{array}{l}\text { In } \\
\text { fr } \\
\text { a. }\end{array}$ & $\begin{array}{l}\text { P } \\
\text { ro } \\
\text { c. }\end{array}$ & $\begin{array}{l}\mathbf{C} \\
\mathbf{R}\end{array}$ & $\begin{array}{l}\text { A } \\
\text { gg } \\
\text { - }\end{array}$ & $\begin{array}{l}\text { Ti } \\
\text { an } \\
\text {. }\end{array}$ & C & & $\begin{array}{l}\mathbf{C} \\
\mathbf{R}\end{array}$ & $\begin{array}{l}\mathbf{E C} \\
>\mathbf{E} \\
\mathbf{C}\end{array}$ & $\begin{array}{l}\text { EC } \\
>\mathbf{n} \\
\text { EC }\end{array}$ & $\begin{array}{l}\mathbf{n E C} \\
>\mathbf{E} \\
\mathrm{C}\end{array}$ & $\begin{array}{l}\mathbf{n E C} \\
>\mathbf{n E} \\
\mathrm{C}\end{array}$ & $\begin{array}{l}\mathbf{C} \\
\mathbf{R}\end{array}$ & $\begin{array}{l}\mathbf{E} \\
\mathbf{W}\end{array}$ & $\begin{array}{l}\mathbf{n} \\
\mathbf{E} \\
\mathbf{W}\end{array}$ & $\begin{array}{l}\mathbf{n} \\
\mathbf{E} \\
\mathbf{n} \\
\mathbf{W}\end{array}$ & $\begin{array}{l}\mathbf{C} \\
\mathbf{R}\end{array}$ & $\begin{array}{l}\mathbf{E} \\
\mathbf{C}\end{array}$ & $\begin{array}{l}\mathbf{n} \\
\mathbf{E} \\
\mathbf{C}\end{array}$ & $\begin{array}{l}\mathbf{C} \\
\mathbf{R}\end{array}$ & $\begin{array}{l}\mathbf{R} \\
\mathbf{E} \\
\mathbf{F}\end{array}$ & 1 & & $\begin{array}{l}\mathbf{n} \\
\mathbf{R} \\
\mathbf{n} \\
\mathbf{E} \\
\mathbf{F}\end{array}$ & $\begin{array}{l}\mathbf{C} \\
\mathbf{R}\end{array}$ & $\begin{array}{l}\mathbf{D} \\
\mathbf{P}\end{array}$ & $\begin{array}{l}\mathbf{n} \\
\mathbf{P}\end{array}$ & $\begin{array}{l}\mathbf{C} \\
\mathbf{R}\end{array}$ & \\
\hline & 8 & $\begin{array}{l}09 \\
1\end{array}$ & $\begin{array}{l}09 \\
1\end{array}$ & $\begin{array}{l}00 \\
0\end{array}$ & $\begin{array}{l}81 \\
8\end{array}$ & $\begin{array}{l}09 \\
1\end{array}$ & 0 & & $\begin{array}{l}00 \\
0\end{array}$ & 7 & 7 & 3 & & $\begin{array}{l}09 \\
1\end{array}$ & $\begin{array}{l}58 \\
2\end{array}$ & $\begin{array}{l}36 \\
7\end{array}$ & $\begin{array}{l}05 \\
1\end{array}$ & $\begin{array}{l}04 \\
6\end{array}$ & $\begin{array}{l}90 \\
0\end{array}$ & $\begin{array}{l}10 \\
0\end{array}$ & $\begin{array}{l}0 \\
0\end{array}$ & $\begin{array}{l}6 \\
2\end{array}$ & 2 & & $\begin{array}{l}06 \\
5\end{array}$ & $\begin{array}{l}02 \\
5\end{array}$ & $\begin{array}{l}90 \\
0\end{array}$ & $\begin{array}{l}10 \\
0\end{array}$ & $\begin{array}{l}00 \\
0\end{array}$ & $\begin{array}{l}02 \\
3\end{array}$ \\
\hline 5 & $\begin{array}{l}0.33 \\
3\end{array}$ & $\begin{array}{l}0 . \\
33 \\
3\end{array}$ & $\begin{array}{l}0 . \\
33 \\
3\end{array}$ & $\begin{array}{l}0 . \\
00 \\
0\end{array}$ & $\begin{array}{l}0 . \\
25 \\
0\end{array}$ & $\begin{array}{l}0 . \\
25 \\
0\end{array}$ & 0 & & $\begin{array}{l}0 . \\
00 \\
0\end{array}$ & $\begin{array}{l}0.20 \\
7\end{array}$ & $\begin{array}{l}0.20 \\
7\end{array}$ & $\begin{array}{l}0.29 \\
3\end{array}$ & 0.293 & $\begin{array}{l}0 . \\
09 \\
1\end{array}$ & $\begin{array}{l}0 . \\
75 \\
1\end{array}$ & $\begin{array}{l}0 . \\
17 \\
8\end{array}$ & $\begin{array}{l}0 . \\
07 \\
0\end{array}$ & $\begin{array}{l}0 . \\
02 \\
5\end{array}$ & $\begin{array}{l}0 . \\
90 \\
0\end{array}$ & $\begin{array}{l}0 . \\
10 \\
0\end{array}$ & $\begin{array}{l}0 \\
0 \\
0\end{array}$ & $\begin{array}{l}0 \\
6 \\
7\end{array}$ & 0 & & $\begin{array}{l}0 . \\
06 \\
3\end{array}$ & $\begin{array}{l}0 . \\
02 \\
5\end{array}$ & $\begin{array}{l}0 . \\
90 \\
0\end{array}$ & $\begin{array}{l}0 . \\
10 \\
0\end{array}$ & $\begin{array}{l}0 . \\
00 \\
0\end{array}$ & $\begin{array}{l}0 . \\
02 \\
0\end{array}$ \\
\hline 6 & $\begin{array}{l}0.10 \\
4\end{array}$ & $\begin{array}{l}0 . \\
12 \\
7\end{array}$ & $\begin{array}{l}0 . \\
76 \\
9\end{array}$ & $\begin{array}{l}0 . \\
03 \\
3\end{array}$ & $\begin{array}{l}0 . \\
09 \\
5\end{array}$ & $\begin{array}{l}0 . \\
25 \\
0\end{array}$ & $\begin{array}{l}6 \\
5\end{array}$ & & $\begin{array}{l}0 . \\
06 \\
3\end{array}$ & $\begin{array}{l}0.25 \\
0\end{array}$ & $\begin{array}{l}0.25 \\
0\end{array}$ & $\begin{array}{l}0.25 \\
0\end{array}$ & 0.250 & $\begin{array}{l}0 . \\
15 \\
5\end{array}$ & $\begin{array}{l}0 . \\
74 \\
3\end{array}$ & $\begin{array}{l}0 . \\
19 \\
4\end{array}$ & $\begin{array}{l}0 . \\
06 \\
3\end{array}$ & $\begin{array}{l}0 . \\
06 \\
1\end{array}$ & $\begin{array}{l}0 . \\
90 \\
0\end{array}$ & $\begin{array}{l}0 . \\
10 \\
0\end{array}$ & $\begin{array}{l}0 \\
0\end{array}$ & $\begin{array}{l}5 \\
2\end{array}$ & 1 & & $\begin{array}{l}0 . \\
05 \\
1\end{array}$ & $\begin{array}{l}0 . \\
04 \\
6\end{array}$ & $\begin{array}{l}0 . \\
90 \\
0\end{array}$ & $\begin{array}{l}0 . \\
10 \\
0\end{array}$ & $\begin{array}{l}0 . \\
00 \\
0\end{array}$ & $\begin{array}{l}0 . \\
05 \\
1\end{array}$ \\
\hline 7 & $\begin{array}{l}0.33 \\
3\end{array}$ & $\begin{array}{l}0 . \\
33 \\
3\end{array}$ & $\begin{array}{l}0 . \\
33 \\
3\end{array}$ & $\begin{array}{l}0 . \\
00 \\
0\end{array}$ & $\begin{array}{l}0 . \\
63 \\
4\end{array}$ & $\begin{array}{l}0 . \\
17 \\
4\end{array}$ & $\begin{array}{l}1 \\
2\end{array}$ & & $\begin{array}{l}0 . \\
00 \\
8\end{array}$ & $\begin{array}{l}0.65 \\
3\end{array}$ & $\begin{array}{l}0.22 \\
8\end{array}$ & $\begin{array}{l}0.06 \\
0\end{array}$ & 0.060 & $\begin{array}{l}0 . \\
09 \\
1\end{array}$ & $\begin{array}{l}0 . \\
79 \\
6\end{array}$ & $\begin{array}{l}0 . \\
12 \\
5\end{array}$ & $\begin{array}{l}0 . \\
07 \\
9\end{array}$ & $\begin{array}{l}0 . \\
04 \\
6\end{array}$ & $\begin{array}{l}0 . \\
90 \\
0\end{array}$ & $\begin{array}{l}0 . \\
10 \\
0\end{array}$ & $\begin{array}{l}0 \\
0 \\
0\end{array}$ & $\begin{array}{l}6 \\
6\end{array}$ & 8 & & $\begin{array}{l}0 . \\
05 \\
8\end{array}$ & $\begin{array}{l}0 . \\
04 \\
6\end{array}$ & $\begin{array}{l}0 . \\
87 \\
5\end{array}$ & $\begin{array}{l}0 . \\
12 \\
5\end{array}$ & $\begin{array}{l}0 . \\
00 \\
0\end{array}$ & $\begin{array}{l}0 . \\
02 \\
7\end{array}$ \\
\hline 8 & $\begin{array}{l}0.33 \\
3\end{array}$ & $\begin{array}{l}0 . \\
33 \\
3\end{array}$ & $\begin{array}{l}0 . \\
33 \\
3\end{array}$ & $\begin{array}{l}0 . \\
00 \\
0\end{array}$ & $\begin{array}{l}0 . \\
48 \\
1\end{array}$ & $\begin{array}{l}0 . \\
05 \\
6\end{array}$ & $\begin{array}{l}4 \\
2\end{array}$ & & $\begin{array}{l}0 . \\
00 \\
1\end{array}$ & $\begin{array}{l}0.75 \\
0\end{array}$ & $\begin{array}{l}0.08 \\
3\end{array}$ & $\begin{array}{l}0.08 \\
3\end{array}$ & 0.083 & $\begin{array}{l}0 . \\
00 \\
0\end{array}$ & $\begin{array}{l}0 . \\
10 \\
2\end{array}$ & $\begin{array}{l}0 . \\
72 \\
6\end{array}$ & $\begin{array}{l}0 . \\
17 \\
2\end{array}$ & $\begin{array}{l}0 . \\
02 \\
5\end{array}$ & $\begin{array}{l}0 . \\
90 \\
0\end{array}$ & $\begin{array}{l}0 . \\
10 \\
0\end{array}$ & $\begin{array}{l}0 \\
0\end{array}$ & $\begin{array}{l}7 \\
5\end{array}$ & 7 & & $\begin{array}{l}0 . \\
05 \\
8\end{array}$ & $\begin{array}{l}0 . \\
10 \\
1\end{array}$ & $\begin{array}{l}0 . \\
87 \\
5\end{array}$ & $\begin{array}{l}0 . \\
12 \\
5\end{array}$ & $\begin{array}{l}0 . \\
00 \\
0\end{array}$ & $\begin{array}{l}0 . \\
01 \\
8\end{array}$ \\
\hline 9 & $\begin{array}{l}0.47 \\
1\end{array}$ & $\begin{array}{l}0 . \\
05 \\
9\end{array}$ & $\begin{array}{l}0 \\
47 \\
1\end{array}$ & $\begin{array}{l}0 . \\
00 \\
0\end{array}$ & $\begin{array}{l}0 . \\
47 \\
1\end{array}$ & $\begin{array}{l}0 . \\
05 \\
9\end{array}$ & 1 & & $\begin{array}{l}0 . \\
00 \\
0\end{array}$ & $\begin{array}{l}0.69 \\
2\end{array}$ & $\begin{array}{l}0.14 \\
0\end{array}$ & $\begin{array}{l}0.07 \\
0\end{array}$ & 0.098 & $\begin{array}{l}0 . \\
09 \\
1\end{array}$ & $\begin{array}{l}0 . \\
20 \\
5\end{array}$ & $\begin{array}{l}0 . \\
72 \\
2\end{array}$ & $\begin{array}{l}0 . \\
07 \\
3\end{array}$ & $\begin{array}{l}0 . \\
10 \\
7\end{array}$ & $\begin{array}{l}0 . \\
90 \\
0\end{array}$ & $\begin{array}{l}0 . \\
10 \\
0\end{array}$ & $\begin{array}{l}0 \\
0\end{array}$ & $\begin{array}{l}6 \\
5\end{array}$ & 7 & & $\begin{array}{l}0 . \\
07 \\
8\end{array}$ & $\begin{array}{l}0 . \\
08 \\
1\end{array}$ & $\begin{array}{l}0 . \\
90 \\
0\end{array}$ & $\begin{array}{l}0 . \\
10 \\
0\end{array}$ & $\begin{array}{l}0 . \\
00 \\
0\end{array}$ & $\begin{array}{l}0 . \\
04 \\
0\end{array}$ \\
\hline 10 & $\begin{array}{l}0.20 \\
0\end{array}$ & $\begin{array}{l}0 . \\
20 \\
0\end{array}$ & $\begin{array}{l}0 . \\
60 \\
0\end{array}$ & $\begin{array}{l}0 . \\
00 \\
0\end{array}$ & $\begin{array}{l}0 . \\
14 \\
9\end{array}$ & $\begin{array}{l}0 . \\
06 \\
6\end{array}$ & 5 & & $\begin{array}{l}0 . \\
06 \\
9\end{array}$ & $\begin{array}{l}0.56 \\
8\end{array}$ & $\begin{array}{l}0.31 \\
9\end{array}$ & $\begin{array}{l}0.06 \\
9\end{array}$ & 0.042 & $\begin{array}{l}0 . \\
06 \\
3\end{array}$ & $\begin{array}{l}0 . \\
73 \\
1\end{array}$ & $\begin{array}{l}0 \\
18 \\
8\end{array}$ & $\begin{array}{l}0 . \\
08 \\
1\end{array}$ & $\begin{array}{l}0 . \\
05 \\
6\end{array}$ & $\begin{array}{l}0 . \\
87 \\
5\end{array}$ & $\begin{array}{l}0 . \\
12 \\
5\end{array}$ & 0 & $\begin{array}{l}6 \\
9\end{array}$ & 9 & & $\begin{array}{l}0 . \\
07 \\
2\end{array}$ & $\begin{array}{l}0 . \\
05 \\
6\end{array}$ & $\begin{array}{l}0 . \\
83 \\
3\end{array}$ & $\begin{array}{l}0 . \\
16 \\
7\end{array}$ & $\begin{array}{l}0 . \\
00 \\
0\end{array}$ & $\begin{array}{l}0 . \\
03 \\
5\end{array}$ \\
\hline 11 & 0.67 & 0. & 0. & 0. & 0. & 0. & 4 & & 0 . & 0.50 & 0.25 & 0.21 & 0.037 & 0. & 0 & 0. & 0. & 0. & 0. & 0. & 0 & 0 & 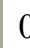 & & 0. & 0. & 0 & 0. & 0 & 0 \\
\hline
\end{tabular}




\begin{tabular}{|c|c|c|c|c|c|c|c|c|c|c|c|c|c|c|c|c|c|c|c|c|c|c|c|c|c|c|c|c|}
\hline \multirow{2}{*}{$\begin{array}{l}\mathbf{P} \\
\text { ar } \\
\text { t. } \\
\text { n } \\
\text { o. }\end{array}$} & \multicolumn{4}{|c|}{ Level 2.1} & \multicolumn{4}{|c|}{ Level 2.2} & \multicolumn{5}{|c|}{ Level 3.1} & \multicolumn{4}{|c|}{ Level 3.2} & \multicolumn{3}{|c|}{ Level 3.3} & \multicolumn{4}{|c|}{ Level 3.4} & \multicolumn{3}{|c|}{ Level 3.5} & \multirow{2}{*}{$\begin{array}{l}\text { A } \\
\text { vg } \\
\dot{C} \\
\text { C }\end{array}$} \\
\hline & $\begin{array}{l}\text { Tec } \\
\text { h./a } \\
\text { gg. }\end{array}$ & $\begin{array}{l}\text { In } \\
\text { fr } \\
\text { a. }\end{array}$ & $\begin{array}{l}\text { P } \\
\text { ro } \\
\text { c. }\end{array}$ & $\begin{array}{l}\mathbf{C} \\
\mathbf{R}\end{array}$ & $\begin{array}{l}\text { A } \\
\text { gg } \\
\text { - }\end{array}$ & $\begin{array}{l}\mathrm{Tr} \\
\text { an } \\
\cdot\end{array}$ & $\begin{array}{l}\text { C } \\
\text { on } \\
\text { t. }\end{array}$ & $\begin{array}{l}\mathbf{C} \\
\mathbf{R}\end{array}$ & $\begin{array}{l}\mathbf{E C} \\
>\mathbf{E} \\
\mathbf{C}\end{array}$ & $\begin{array}{l}\text { EC } \\
>\text { n } \\
\text { EC }\end{array}$ & $\begin{array}{l}\mathrm{nEC} \\
>\mathrm{E} \\
\mathrm{C}\end{array}$ & $\begin{array}{l}\mathbf{n E C} \\
>\mathbf{n E} \\
\mathrm{C}\end{array}$ & $\begin{array}{l}\mathbf{C} \\
\mathbf{R}\end{array}$ & $\begin{array}{l}\mathbf{E} \\
\mathbf{W}\end{array}$ & $\begin{array}{l}\mathbf{n} \\
\mathbf{E} \\
\mathbf{W}\end{array}$ & $\begin{array}{l}\mathbf{n} \\
\mathbf{E} \\
\mathbf{n} \\
\mathbf{W}\end{array}$ & $\begin{array}{l}\mathbf{C} \\
\mathbf{R}\end{array}$ & $\begin{array}{l}\mathbf{E} \\
\mathbf{C}\end{array}$ & $\begin{array}{l}\mathbf{n} \\
\mathbf{E} \\
\mathbf{C}\end{array}$ & $\begin{array}{l}\mathbf{C} \\
\mathbf{R}\end{array}$ & $\begin{array}{l}\mathbf{R} \\
\mathbf{E} \\
\mathbf{F}\end{array}$ & $\begin{array}{l}\mathbf{n} \\
\mathbf{R} \\
\mathbf{E} \\
\mathbf{F}\end{array}$ & $\begin{array}{l}\mathbf{n} \\
\mathbf{R} \\
\mathbf{n} \\
\mathbf{E} \\
\mathbf{F}\end{array}$ & $\begin{array}{l}\mathbf{C} \\
\mathbf{R}\end{array}$ & $\begin{array}{l}\mathbf{D} \\
\mathbf{P}\end{array}$ & $\begin{array}{l}\mathbf{n} \\
\mathbf{P}\end{array}$ & $\begin{array}{l}\mathbf{C} \\
\mathbf{R}\end{array}$ & \\
\hline & 8 & $\begin{array}{l}14 \\
2\end{array}$ & $\begin{array}{l}17 \\
9\end{array}$ & $\begin{array}{l}04 \\
6\end{array}$ & $\begin{array}{l}12 \\
9\end{array}$ & $\begin{array}{l}08 \\
5\end{array}$ & $\begin{array}{l}78 \\
5\end{array}$ & $\begin{array}{l}06 \\
6\end{array}$ & 1 & 1 & 1 & & $\begin{array}{l}06 \\
8\end{array}$ & $\begin{array}{l}79 \\
8\end{array}$ & $\begin{array}{l}13 \\
8\end{array}$ & $\begin{array}{l}06 \\
4\end{array}$ & $\begin{array}{l}09 \\
3\end{array}$ & $\begin{array}{l}85 \\
7\end{array}$ & $\begin{array}{l}14 \\
3\end{array}$ & $\begin{array}{l}00 \\
0\end{array}$ & $\begin{array}{l}65 \\
5\end{array}$ & $\begin{array}{l}29 \\
0\end{array}$ & $\begin{array}{l}05 \\
5\end{array}$ & $\begin{array}{l}06 \\
9\end{array}$ & $\begin{array}{l}85 \\
7\end{array}$ & $\begin{array}{l}14 \\
3\end{array}$ & $\begin{array}{l}00 \\
0\end{array}$ & $\begin{array}{l}04 \\
9\end{array}$ \\
\hline $\begin{array}{l}\mathrm{M} \\
\text { ea } \\
\mathrm{n}\end{array}$ & $\begin{array}{l}0.22 \\
0\end{array}$ & $\begin{array}{l}0 . \\
21 \\
1\end{array}$ & $\begin{array}{l}0 . \\
37 \\
4\end{array}$ & - & $\begin{array}{l}0 . \\
28 \\
0\end{array}$ & $\begin{array}{l}0 . \\
12 \\
3\end{array}$ & $\begin{array}{l}0 . \\
35 \\
6\end{array}$ & - & $\begin{array}{l}0.42 \\
8\end{array}$ & $\begin{array}{l}0.16 \\
7\end{array}$ & $\begin{array}{l}0.16 \\
9\end{array}$ & 0.090 & - & $\begin{array}{l}0 . \\
60 \\
1\end{array}$ & $\begin{array}{l}0 . \\
21 \\
6\end{array}$ & $\begin{array}{l}0 . \\
07 \\
4\end{array}$ & - & $\begin{array}{l}0 . \\
89 \\
1\end{array}$ & $\begin{array}{l}0 . \\
10 \\
8\end{array}$ & & $\begin{array}{l}0 . \\
68 \\
1\end{array}$ & $\begin{array}{l}0 . \\
22 \\
6\end{array}$ & $\begin{array}{l}0 . \\
06 \\
8\end{array}$ & - & $\begin{array}{l}0 . \\
88 \\
3\end{array}$ & $\begin{array}{l}0 . \\
11 \\
5\end{array}$ & - & - \\
\hline $\begin{array}{l}\text { A } \\
\text { vg } \\
.\end{array}$ & $\begin{array}{l}0.31 \\
5\end{array}$ & $\begin{array}{l}0 . \\
25 \\
3\end{array}$ & $\begin{array}{l}0 . \\
43 \\
1\end{array}$ & $\begin{array}{l}0 . \\
01 \\
7\end{array}$ & $\begin{array}{l}0 . \\
39 \\
1\end{array}$ & $\begin{array}{l}0 . \\
15 \\
9\end{array}$ & $\begin{array}{l}0 . \\
45 \\
0\end{array}$ & $\begin{array}{l}0 . \\
02 \\
1\end{array}$ & $\begin{array}{l}0.46 \\
7\end{array}$ & $\begin{array}{l}0.19 \\
2\end{array}$ & $\begin{array}{l}0.22 \\
3\end{array}$ & 0.118 & $\begin{array}{l}0 . \\
07 \\
2\end{array}$ & $\begin{array}{l}0 . \\
67 \\
1\end{array}$ & $\begin{array}{l}0 . \\
25 \\
1\end{array}$ & $\begin{array}{l}0 . \\
07 \\
8\end{array}$ & $\begin{array}{l}0 . \\
05 \\
7\end{array}$ & $\begin{array}{l}0 . \\
89 \\
2\end{array}$ & $\begin{array}{l}0 . \\
10 \\
8\end{array}$ & $\begin{array}{l}0 . \\
00 \\
0\end{array}$ & $\begin{array}{l}0 . \\
68 \\
5\end{array}$ & $\begin{array}{l}0 . \\
24 \\
1\end{array}$ & $\begin{array}{l}0 . \\
06 \\
9\end{array}$ & $\begin{array}{l}0 . \\
05 \\
0\end{array}$ & $\begin{array}{l}0 . \\
88 \\
3\end{array}$ & $\begin{array}{l}0 . \\
11 \\
7\end{array}$ & $\begin{array}{l}0 . \\
00 \\
0\end{array}$ & $\begin{array}{l}0 . \\
03 \\
5\end{array}$ \\
\hline $\begin{array}{l}\text { St } \\
\text { d. } \\
\text { de } \\
\text { v. }\end{array}$ & $\begin{array}{l}0.25 \\
6\end{array}$ & $\begin{array}{l}0 . \\
14 \\
2\end{array}$ & $\begin{array}{l}0 . \\
20 \\
5\end{array}$ & $\begin{array}{l}0 . \\
03 \\
4\end{array}$ & $\begin{array}{l}0 . \\
29 \\
4\end{array}$ & $\begin{array}{l}0 . \\
13 \\
2\end{array}$ & $\begin{array}{l}0 . \\
25 \\
7\end{array}$ & $\begin{array}{l}0 . \\
03 \\
1\end{array}$ & $\begin{array}{l}0.19 \\
4\end{array}$ & $\begin{array}{l}0.09 \\
3\end{array}$ & $\begin{array}{l}0.16 \\
6\end{array}$ & 0.095 & $\begin{array}{l}0 . \\
04 \\
0\end{array}$ & $\begin{array}{l}0 . \\
20 \\
9\end{array}$ & $\begin{array}{l}0 . \\
17 \\
9\end{array}$ & $\begin{array}{l}0 . \\
03 \\
4\end{array}$ & $\begin{array}{l}0 . \\
02 \\
5\end{array}$ & $\begin{array}{l}0 . \\
01 \\
5\end{array}$ & $\begin{array}{l}0 . \\
01 \\
5\end{array}$ & $\begin{array}{l}0 . \\
00 \\
0\end{array}$ & $\begin{array}{l}0 . \\
07 \\
0\end{array}$ & $\begin{array}{l}0 . \\
07 \\
8\end{array}$ & $\begin{array}{l}0 . \\
01 \\
4\end{array}$ & $\begin{array}{l}0 . \\
02 \\
8\end{array}$ & $\begin{array}{l}0 . \\
02 \\
2\end{array}$ & $\begin{array}{l}0 . \\
02 \\
2\end{array}$ & $\begin{array}{l}0 . \\
00 \\
0\end{array}$ & $\begin{array}{l}0 . \\
01 \\
7\end{array}$ \\
\hline
\end{tabular}


Table 6.

AHP results for the waste treatment life cycle phase (onsite)

\begin{tabular}{|c|c|c|c|c|c|c|c|c|c|c|c|c|c|c|c|c|c|c|c|c|c|c|c|c|c|c|}
\hline \multirow{2}{*}{$\begin{array}{l}\text { Pa } \\
\text { rt. } \\
\text { no. }\end{array}$} & \multicolumn{4}{|c|}{ Level 2.1} & \multicolumn{4}{|c|}{ Level 2.2} & \multicolumn{4}{|c|}{ Level 3.1} & \multicolumn{4}{|c|}{ Level 3.2} & \multicolumn{3}{|c|}{ Level 3.3} & \multicolumn{3}{|c|}{ Level 3.4} & \multicolumn{3}{|c|}{ Level 3.5} & \multirow{2}{*}{$\begin{array}{l}\text { Av } \\
\text { g. } \\
\text { C } \\
\text { R }\end{array}$} \\
\hline & $\begin{array}{l}\text { Tech. } \\
\text { /Eq. }\end{array}$ & $\begin{array}{l}\text { Inf } \\
\text { ra. }\end{array}$ & $\begin{array}{l}\text { Pr } \\
\text { oc. }\end{array}$ & $\begin{array}{l}\mathbf{C} \\
\mathbf{R}\end{array}$ & $\begin{array}{l}\mathbf{E q} \\
\text {. }\end{array}$ & $\begin{array}{l}\text { Tr } \\
\text { an. }\end{array}$ & $\begin{array}{l}\text { Co } \\
\text { nt. }\end{array}$ & $\begin{array}{l}\mathbf{C} \\
\mathbf{R}\end{array}$ & $\begin{array}{l}\text { SA } \\
\text { SSI } \\
-E\end{array}$ & $\begin{array}{l}\text { SA } \\
\text { SSI } \\
\text {-M }\end{array}$ & $\begin{array}{l}\mathbf{O} \\
\mathbf{A} \\
\mathbf{B}\end{array}$ & $\begin{array}{l}\mathbf{C} \\
\mathbf{R}\end{array}$ & $\begin{array}{l}\mathbf{E} \\
\mathbf{W} \\
\mathbf{T}\end{array}$ & $\begin{array}{l}\mathbf{G} \\
\mathbf{T}\end{array}$ & IT & $\begin{array}{l}\mathbf{C} \\
\mathbf{R}\end{array}$ & $\begin{array}{l}\mathbf{E} \\
\mathbf{C}\end{array}$ & $\begin{array}{l}\mathbf{n E} \\
\mathrm{C}\end{array}$ & $\begin{array}{l}\mathbf{C} \\
\mathbf{R}\end{array}$ & ES & $\begin{array}{l}\mathbf{n E} \\
\mathrm{S}\end{array}$ & $\begin{array}{l}\mathbf{C} \\
\mathbf{R}\end{array}$ & $\begin{array}{l}\mathbf{D} \\
\mathbf{P}\end{array}$ & $\mathbf{n P}$ & $\begin{array}{l}\mathbf{C} \\
\mathbf{R}\end{array}$ & \\
\hline \multicolumn{27}{|c|}{ South African workshop } \\
\hline 1 & 0.333 & $\begin{array}{l}0.3 \\
33\end{array}$ & $\begin{array}{l}0.3 \\
33\end{array}$ & $\begin{array}{l}0.0 \\
00\end{array}$ & $\begin{array}{l}0.4 \\
74\end{array}$ & $\begin{array}{l}0.0 \\
53\end{array}$ & $\begin{array}{l}0.4 \\
74\end{array}$ & $\begin{array}{l}0.0 \\
00\end{array}$ & $\begin{array}{l}0.76 \\
2\end{array}$ & $\begin{array}{l}0.19 \\
0\end{array}$ & $\begin{array}{l}0.0 \\
48\end{array}$ & $\begin{array}{l}0.4 \\
31\end{array}$ & $\begin{array}{l}0.8 \\
14\end{array}$ & $\begin{array}{l}0.1 \\
14\end{array}$ & $\begin{array}{l}0.0 \\
72\end{array}$ & $\begin{array}{l}0.0 \\
46\end{array}$ & $\begin{array}{l}0.9 \\
00\end{array}$ & $\begin{array}{l}0.1 \\
00\end{array}$ & $\begin{array}{l}0.0 \\
00\end{array}$ & $\begin{array}{l}0.9 \\
00\end{array}$ & $\begin{array}{l}0.1 \\
00\end{array}$ & $\begin{array}{l}0.0 \\
00\end{array}$ & $\begin{array}{l}0.9 \\
00\end{array}$ & $\begin{array}{l}0.1 \\
00\end{array}$ & $\begin{array}{l}0.0 \\
00\end{array}$ & $\begin{array}{l}0.0 \\
68\end{array}$ \\
\hline 2 & 0.550 & $\begin{array}{l}0.2 \\
10\end{array}$ & $\begin{array}{l}0.2 \\
40\end{array}$ & $\begin{array}{l}0.0 \\
16\end{array}$ & $\begin{array}{l}0.6 \\
34\end{array}$ & $\begin{array}{l}0.1 \\
74\end{array}$ & $\begin{array}{l}0.1 \\
92\end{array}$ & $\begin{array}{l}0.0 \\
08\end{array}$ & $\begin{array}{l}0.78 \\
0\end{array}$ & $\begin{array}{l}0.17 \\
0\end{array}$ & $\begin{array}{l}0.0 \\
50\end{array}$ & $\begin{array}{l}0.2 \\
75\end{array}$ & $\begin{array}{l}0.7 \\
75\end{array}$ & $\begin{array}{l}0.1 \\
78\end{array}$ & $\begin{array}{l}0.0 \\
47\end{array}$ & $\begin{array}{l}0.3 \\
30\end{array}$ & $\begin{array}{l}0.9 \\
00\end{array}$ & $\begin{array}{l}0.1 \\
00\end{array}$ & $\begin{array}{l}0.0 \\
00\end{array}$ & $\begin{array}{l}0.8 \\
75\end{array}$ & $\begin{array}{l}0.1 \\
25\end{array}$ & $\begin{array}{l}0.0 \\
00\end{array}$ & $\begin{array}{l}0.8 \\
89\end{array}$ & $\begin{array}{l}0.1 \\
11\end{array}$ & $\begin{array}{l}0.0 \\
00\end{array}$ & $\begin{array}{l}0.0 \\
90\end{array}$ \\
\hline 3 & 0.667 & $\begin{array}{l}0.1 \\
67\end{array}$ & $\begin{array}{l}0.1 \\
67\end{array}$ & $\begin{array}{l}0.0 \\
00\end{array}$ & $\begin{array}{l}0.6 \\
67\end{array}$ & $\begin{array}{l}0.1 \\
67\end{array}$ & $\begin{array}{l}0.1 \\
67\end{array}$ & $\begin{array}{l}0.0 \\
00\end{array}$ & $\begin{array}{l}0.76 \\
2\end{array}$ & $\begin{array}{l}0.19 \\
0\end{array}$ & $\begin{array}{l}0.0 \\
48\end{array}$ & $\begin{array}{l}0.4 \\
31\end{array}$ & $\begin{array}{l}0.7 \\
66\end{array}$ & $\begin{array}{l}0.1 \\
58\end{array}$ & $\begin{array}{l}0.0 \\
76\end{array}$ & $\begin{array}{l}0.1 \\
17\end{array}$ & $\begin{array}{l}0.9 \\
00\end{array}$ & $\begin{array}{l}0.1 \\
00\end{array}$ & $\begin{array}{l}0.0 \\
00\end{array}$ & $\begin{array}{l}0.8 \\
75\end{array}$ & $\begin{array}{l}0.1 \\
25\end{array}$ & $\begin{array}{l}0.0 \\
00\end{array}$ & $\begin{array}{l}0.8 \\
33\end{array}$ & $\begin{array}{l}0.1 \\
67\end{array}$ & $\begin{array}{l}0.0 \\
00\end{array}$ & $\begin{array}{l}0.0 \\
78\end{array}$ \\
\hline 4 & 0.300 & $\begin{array}{l}0.6 \\
00\end{array}$ & $\begin{array}{l}0.1 \\
00\end{array}$ & $\begin{array}{l}0.0 \\
00\end{array}$ & $\begin{array}{l}0.3 \\
09\end{array}$ & $\begin{array}{l}0.1 \\
09\end{array}$ & $\begin{array}{l}0.5 \\
82\end{array}$ & $\begin{array}{l}0.0 \\
03\end{array}$ & $\begin{array}{l}0.73 \\
6\end{array}$ & $\begin{array}{l}0.19 \\
9\end{array}$ & $\begin{array}{l}0.0 \\
65\end{array}$ & $\begin{array}{l}0.2 \\
07\end{array}$ & $\begin{array}{l}0.6 \\
26\end{array}$ & $\begin{array}{l}0.3 \\
01\end{array}$ & $\begin{array}{l}0.0 \\
72\end{array}$ & $\begin{array}{l}0.0 \\
01\end{array}$ & $\begin{array}{l}0.9 \\
00\end{array}$ & $\begin{array}{l}0.1 \\
00\end{array}$ & $\begin{array}{l}0.0 \\
00\end{array}$ & $\begin{array}{l}0.8 \\
75\end{array}$ & $\begin{array}{l}0.1 \\
25\end{array}$ & $\begin{array}{l}0.0 \\
00\end{array}$ & $\begin{array}{l}0.8 \\
89\end{array}$ & $\begin{array}{l}0.1 \\
11\end{array}$ & $\begin{array}{l}0.0 \\
00\end{array}$ & $\begin{array}{l}0.0 \\
30\end{array}$ \\
\hline 5 & 0.333 & $\begin{array}{l}0.3 \\
33\end{array}$ & $\begin{array}{l}0.3 \\
33\end{array}$ & $\begin{array}{l}0.0 \\
00\end{array}$ & $\begin{array}{l}0.3 \\
33\end{array}$ & $\begin{array}{l}0.3 \\
33\end{array}$ & $\begin{array}{l}0.3 \\
33\end{array}$ & $\begin{array}{l}0.0 \\
00\end{array}$ & $\begin{array}{l}0.78 \\
0\end{array}$ & $\begin{array}{l}0.17 \\
0\end{array}$ & $\begin{array}{l}0.0 \\
50\end{array}$ & $\begin{array}{l}0.2 \\
75\end{array}$ & $\begin{array}{l}0.7 \\
85\end{array}$ & $\begin{array}{l}0.1 \\
61\end{array}$ & $\begin{array}{l}0.0 \\
53\end{array}$ & $\begin{array}{l}0.2 \\
18\end{array}$ & $\begin{array}{l}0.8 \\
89\end{array}$ & $\begin{array}{l}0.1 \\
11\end{array}$ & $\begin{array}{l}0.0 \\
00\end{array}$ & $\begin{array}{l}0.8 \\
75\end{array}$ & $\begin{array}{l}0.1 \\
25\end{array}$ & $\begin{array}{l}0.0 \\
00\end{array}$ & $\begin{array}{l}0.9 \\
00\end{array}$ & $\begin{array}{l}0.1 \\
00\end{array}$ & $\begin{array}{l}0.0 \\
00\end{array}$ & $\begin{array}{l}0.0 \\
70\end{array}$ \\
\hline 6 & 0.333 & $\begin{array}{l}0.3 \\
33\end{array}$ & $\begin{array}{l}0.3 \\
33\end{array}$ & $\begin{array}{l}0.0 \\
00\end{array}$ & $\begin{array}{l}0.3 \\
33\end{array}$ & $\begin{array}{l}0.3 \\
33\end{array}$ & $\begin{array}{l}0.3 \\
33\end{array}$ & $\begin{array}{l}0.0 \\
00\end{array}$ & $\begin{array}{l}0.78 \\
5\end{array}$ & $\begin{array}{l}0.16 \\
1\end{array}$ & $\begin{array}{l}0.0 \\
53\end{array}$ & $\begin{array}{l}0.2 \\
18\end{array}$ & $\begin{array}{l}0.8 \\
04\end{array}$ & $\begin{array}{l}0.1 \\
22\end{array}$ & $\begin{array}{l}0.0 \\
74\end{array}$ & $\begin{array}{l}0.0 \\
32\end{array}$ & $\begin{array}{l}0.9 \\
00\end{array}$ & $\begin{array}{l}0.1 \\
00\end{array}$ & $\begin{array}{l}0.0 \\
00\end{array}$ & $\begin{array}{l}0.9 \\
00\end{array}$ & $\begin{array}{l}0.1 \\
00\end{array}$ & $\begin{array}{l}0.0 \\
00\end{array}$ & $\begin{array}{l}0.9 \\
00\end{array}$ & $\begin{array}{l}0.1 \\
00\end{array}$ & $\begin{array}{l}0.0 \\
00\end{array}$ & $\begin{array}{l}0.0 \\
36\end{array}$ \\
\hline 7 & 0.333 & $\begin{array}{l}0.3 \\
33\end{array}$ & $\begin{array}{l}0.3 \\
33\end{array}$ & $\begin{array}{l}0.0 \\
00\end{array}$ & $\begin{array}{l}0.4 \\
67\end{array}$ & $\begin{array}{l}0.0 \\
67\end{array}$ & $\begin{array}{l}0.4 \\
67\end{array}$ & $\begin{array}{l}0.0 \\
00\end{array}$ & $\begin{array}{l}0.73 \\
6\end{array}$ & $\begin{array}{l}0.21 \\
1\end{array}$ & $\begin{array}{l}0.0 \\
54\end{array}$ & $\begin{array}{l}0.6 \\
26\end{array}$ & $\begin{array}{l}0.3 \\
33\end{array}$ & $\begin{array}{l}0.3 \\
33\end{array}$ & $\begin{array}{l}0.3 \\
33\end{array}$ & $\begin{array}{l}0.0 \\
00\end{array}$ & $\begin{array}{l}0.9 \\
00\end{array}$ & $\begin{array}{l}0.1 \\
00\end{array}$ & $\begin{array}{l}0.0 \\
00\end{array}$ & $\begin{array}{l}0.8 \\
33\end{array}$ & $\begin{array}{l}0.1 \\
67\end{array}$ & $\begin{array}{l}0.0 \\
00\end{array}$ & $\begin{array}{l}0.8 \\
89\end{array}$ & $\begin{array}{l}0.1 \\
11\end{array}$ & $\begin{array}{l}0.0 \\
00\end{array}$ & $\begin{array}{l}0.0 \\
89\end{array}$ \\
\hline 8 & 0.333 & $\begin{array}{l}0.3 \\
33\end{array}$ & $\begin{array}{l}0.3 \\
33\end{array}$ & $\begin{array}{l}0.0 \\
00\end{array}$ & $\begin{array}{l}0.4 \\
00\end{array}$ & $\begin{array}{l}0.2 \\
00\end{array}$ & $\begin{array}{l}0.4 \\
00\end{array}$ & $\begin{array}{l}0.0 \\
00\end{array}$ & $\begin{array}{l}0.47 \\
1\end{array}$ & $\begin{array}{l}0.47 \\
1\end{array}$ & $\begin{array}{l}0.0 \\
59\end{array}$ & $\begin{array}{l}0.0 \\
00\end{array}$ & $\begin{array}{l}0.7 \\
61\end{array}$ & $\begin{array}{l}0.1 \\
66\end{array}$ & $\begin{array}{l}0.0 \\
73\end{array}$ & $\begin{array}{l}0.0 \\
63\end{array}$ & $\begin{array}{l}0.8 \\
89\end{array}$ & $\begin{array}{l}0.1 \\
11\end{array}$ & $\begin{array}{l}0.0 \\
00\end{array}$ & $\begin{array}{l}0.8 \\
89\end{array}$ & $\begin{array}{l}0.1 \\
11\end{array}$ & $\begin{array}{l}0.0 \\
00\end{array}$ & $\begin{array}{l}0.9 \\
00\end{array}$ & $\begin{array}{l}0.1 \\
00\end{array}$ & $\begin{array}{l}0.0 \\
00\end{array}$ & $\begin{array}{l}0.0 \\
09\end{array}$ \\
\hline
\end{tabular}




\begin{tabular}{|c|c|c|c|c|c|c|c|c|c|c|c|c|c|c|c|c|c|c|c|c|c|c|c|c|c|c|c|}
\hline \multirow{2}{*}{$\begin{array}{l}\text { Pa } \\
\text { rt. } \\
\text { no. }\end{array}$} & \multicolumn{4}{|c|}{ Level 2.1} & \multicolumn{4}{|c|}{ Level 2.2} & \multicolumn{4}{|c|}{ Level 3.1} & \multicolumn{5}{|c|}{ Level 3.2} & \multicolumn{3}{|c|}{ Level 3.3} & \multicolumn{3}{|c|}{ Level 3.4} & \multicolumn{3}{|c|}{ Level 3.5} & \multirow{2}{*}{$\begin{array}{l}\text { Av } \\
\text { g. } \\
\text { C } \\
\text { R }\end{array}$} \\
\hline & $\begin{array}{l}\text { Tech. } \\
\text { /Eq. }\end{array}$ & $\begin{array}{l}\text { Inf } \\
\text { ra. }\end{array}$ & $\begin{array}{l}\text { Pr } \\
\text { oc. }\end{array}$ & $\begin{array}{l}\mathbf{C} \\
\mathbf{R}\end{array}$ & $\begin{array}{l}\text { Eq } \\
.\end{array}$ & $\begin{array}{l}\text { Tr } \\
\text { an. }\end{array}$ & $\begin{array}{l}\text { Co } \\
\text { nt. }\end{array}$ & $\begin{array}{l}\mathbf{C} \\
\mathbf{R}\end{array}$ & \begin{tabular}{|l} 
SA \\
SSI \\
$-E$
\end{tabular} & $\begin{array}{l}\text { SA } \\
\text { SSI } \\
\text {-M }\end{array}$ & $\begin{array}{l}\text { O } \\
\text { A } \\
\text { B }\end{array}$ & $\begin{array}{l}C \\
\text { R }\end{array}$ & 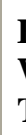 & & $\begin{array}{l}G \\
T\end{array}$ & IT & $\begin{array}{l}\mathbf{C} \\
\mathbf{R}\end{array}$ & $\begin{array}{l}\text { E } \\
\mathbf{C}\end{array}$ & $\begin{array}{l}\mathbf{n E} \\
\mathbf{C}\end{array}$ & $\begin{array}{l}\mathbf{C} \\
\mathbf{R}\end{array}$ & ES & $\begin{array}{l}\mathbf{n E} \\
\mathbf{S}\end{array}$ & $\begin{array}{l}\mathbf{C} \\
\mathbf{R}\end{array}$ & $\begin{array}{l}\mathbf{D} \\
\mathbf{P}\end{array}$ & $\mathbf{n P}$ & $\begin{array}{l}\mathbf{C} \\
\mathbf{R}\end{array}$ & \\
\hline 9 & 0.251 & $\begin{array}{l}0.6 \\
73\end{array}$ & $\begin{array}{l}0.0 \\
75\end{array}$ & $\begin{array}{l}0.1 \\
41\end{array}$ & $\begin{array}{l}0.2 \\
40\end{array}$ & $\begin{array}{l}0.2 \\
10\end{array}$ & $\begin{array}{l}0.5 \\
50\end{array}$ & $\begin{array}{l}0.0 \\
16\end{array}$ & $\begin{array}{l}0.64 \\
9\end{array}$ & $\begin{array}{l}0.27 \\
9\end{array}$ & $\begin{array}{l}0 . \\
72\end{array}$ & $\begin{array}{l}0 . \\
56\end{array}$ & & & $\begin{array}{l}0.2 \\
86\end{array}$ & $\begin{array}{l}0.1 \\
43\end{array}$ & $\begin{array}{l}0.0 \\
00\end{array}$ & $\begin{array}{l}0.8 \\
00\end{array}$ & $\begin{array}{l}0.2 \\
00\end{array}$ & $\begin{array}{l}0.0 \\
00\end{array}$ & $\begin{array}{l}0.8 \\
57\end{array}$ & $\begin{array}{l}0.1 \\
43\end{array}$ & $\begin{array}{l}0.0 \\
00\end{array}$ & $\begin{array}{l}0.8 \\
75\end{array}$ & $\begin{array}{l}0.1 \\
25\end{array}$ & $\begin{array}{l}0.0 \\
00\end{array}$ & $\begin{array}{l}0.0 \\
30\end{array}$ \\
\hline 10 & 0.602 & $\begin{array}{l}0.3 \\
24\end{array}$ & $\begin{array}{l}0.0 \\
75\end{array}$ & $\begin{array}{l}0.2 \\
01\end{array}$ & $\begin{array}{l}0.6 \\
33\end{array}$ & $\begin{array}{l}0.0 \\
63\end{array}$ & $\begin{array}{l}0.3 \\
04\end{array}$ & $\begin{array}{l}0.1 \\
17\end{array}$ & $\begin{array}{l}0.77 \\
2\end{array}$ & $\begin{array}{l}0.17 \\
3\end{array}$ & $\begin{array}{l}0 . \\
55\end{array}$ & $\begin{array}{l}0 . \\
80\end{array}$ & & & $\begin{array}{l}0.1 \\
88\end{array}$ & $\begin{array}{l}0.0 \\
81\end{array}$ & $\begin{array}{l}0.0 \\
56\end{array}$ & $\begin{array}{l}0.9 \\
00\end{array}$ & $\begin{array}{l}0.1 \\
00\end{array}$ & $\begin{array}{l}0.0 \\
00\end{array}$ & $\begin{array}{l}0.8 \\
33\end{array}$ & $\begin{array}{l}0.1 \\
67\end{array}$ & $\begin{array}{l}0.0 \\
00\end{array}$ & $\begin{array}{l}0.9 \\
00\end{array}$ & $\begin{array}{l}0.1 \\
00\end{array}$ & $\begin{array}{l}0.0 \\
00\end{array}$ & $\begin{array}{l}0.0 \\
79\end{array}$ \\
\hline 11 & 0.400 & $\begin{array}{l}0.4 \\
00\end{array}$ & $\begin{array}{l}0.2 \\
00\end{array}$ & $\begin{array}{l}0.4 \\
31\end{array}$ & $\begin{array}{l}0.1 \\
35\end{array}$ & $\begin{array}{l}0.1 \\
55\end{array}$ & $\begin{array}{l}0.7 \\
10\end{array}$ & $\begin{array}{l}0.0 \\
16\end{array}$ & $\begin{array}{l}0.77 \\
4\end{array}$ & $\begin{array}{l}0.17 \\
0\end{array}$ & $\begin{array}{l}0 . \\
56\end{array}$ & $\begin{array}{l}0 . \\
16\end{array}$ & & & $\begin{array}{l}0.3 \\
57\end{array}$ & $\begin{array}{l}0.0 \\
54\end{array}$ & $\begin{array}{l}0.0 \\
32\end{array}$ & $\begin{array}{l}0.9 \\
00\end{array}$ & $\begin{array}{l}0.1 \\
00\end{array}$ & $\begin{array}{l}0.0 \\
00\end{array}$ & $\begin{array}{l}0.8 \\
89\end{array}$ & $\begin{array}{l}0.1 \\
11\end{array}$ & $\begin{array}{l}0.0 \\
00\end{array}$ & $\begin{array}{l}0.9 \\
00\end{array}$ & $\begin{array}{l}0.1 \\
00\end{array}$ & $\begin{array}{l}0.0 \\
00\end{array}$ & $\begin{array}{l}0.1 \\
28\end{array}$ \\
\hline $\begin{array}{l}\mathrm{M} \\
\mathrm{ea} \\
\mathrm{n}\end{array}$ & 0.382 & \begin{tabular}{|l|}
0.3 \\
10
\end{tabular} & $\begin{array}{l}0.2 \\
52\end{array}$ & - & $\begin{array}{l}0.3 \\
83\end{array}$ & $\begin{array}{l}0.1 \\
43\end{array}$ & $\begin{array}{l}0.3 \\
77\end{array}$ & - & $\begin{array}{l}0.55 \\
3\end{array}$ & $\begin{array}{l}0.36 \\
3\end{array}$ & $\begin{array}{l}0 . \\
65\end{array}$ & - & & & $\begin{array}{l}0.2 \\
07\end{array}$ & $\begin{array}{l}0.0 \\
91\end{array}$ & - & $\begin{array}{l}0.8 \\
88\end{array}$ & \begin{tabular}{|l}
0.1 \\
09
\end{tabular} & - & $\begin{array}{l}0.8 \\
73\end{array}$ & $\begin{array}{l}0.1 \\
25\end{array}$ & - & $\begin{array}{l}0.8 \\
88\end{array}$ & $\begin{array}{l}0.1 \\
10\end{array}$ & - & - \\
\hline $\begin{array}{l}\text { Av } \\
\text { g. }\end{array}$ & 0.398 & $\begin{array}{l}0.3 \\
30\end{array}$ & $\begin{array}{l}0.2 \\
72\end{array}$ & $\begin{array}{l}0.0 \\
02\end{array}$ & $\begin{array}{l}0.4 \\
20\end{array}$ & $\begin{array}{l}0.1 \\
69\end{array}$ & $\begin{array}{l}0.4 \\
10\end{array}$ & $\begin{array}{l}0.0 \\
15\end{array}$ & $\begin{array}{l}0.56 \\
0\end{array}$ & $\begin{array}{l}0.37 \\
5\end{array}$ & $\begin{array}{l}0 . \\
66\end{array}$ & $\begin{array}{l}0 . \\
2 \varepsilon\end{array}$ & & & $\begin{array}{l}0.2 \\
25\end{array}$ & $\begin{array}{l}0.1 \\
09\end{array}$ & $\begin{array}{l}0.0 \\
39\end{array}$ & $\begin{array}{l}0.8 \\
89\end{array}$ & $\begin{array}{l}0.1 \\
11\end{array}$ & $\begin{array}{l}0.0 \\
00\end{array}$ & $\begin{array}{l}0.8 \\
73\end{array}$ & $\begin{array}{l}0.1 \\
27\end{array}$ & $\begin{array}{l}0.0 \\
00\end{array}$ & $\begin{array}{l}0.8 \\
89\end{array}$ & $\begin{array}{l}0.1 \\
11\end{array}$ & $\begin{array}{l}0.0 \\
00\end{array}$ & $\begin{array}{l}0.0 \\
64\end{array}$ \\
\hline $\begin{array}{l}\text { St } \\
\text { d. } \\
\text { de } \\
\text { v. }\end{array}$ & 0.134 & $\begin{array}{l}0.1 \\
28\end{array}$ & $\begin{array}{l}0.0 \\
93\end{array}$ & $\begin{array}{l}0.0 \\
06\end{array}$ & $\begin{array}{l}0.1 \\
73\end{array}$ & $\begin{array}{l}0.0 \\
98\end{array}$ & $\begin{array}{l}0.1 \\
66\end{array}$ & $\begin{array}{l}0.0 \\
35\end{array}$ & $\begin{array}{l}0.12 \\
6\end{array}$ & $\begin{array}{l}0.13 \\
6\end{array}$ & $\begin{array}{l}0 . \\
09\end{array}$ & $\begin{array}{l}0 . \\
40\end{array}$ & & & $\begin{array}{l}0.0 \\
94\end{array}$ & $\begin{array}{l}0.0 \\
88\end{array}$ & $\begin{array}{l}0.0 \\
38\end{array}$ & $\begin{array}{l}0.0 \\
30\end{array}$ & $\begin{array}{l}0.0 \\
30\end{array}$ & $\begin{array}{l}0.0 \\
00\end{array}$ & $\begin{array}{l}0.0 \\
23\end{array}$ & $\begin{array}{l}0.0 \\
23\end{array}$ & \begin{tabular}{|l}
0.0 \\
00
\end{tabular} & $\begin{array}{l}0.0 \\
20\end{array}$ & $\begin{array}{l}0.0 \\
20\end{array}$ & $\begin{array}{l}0.0 \\
00\end{array}$ & $\begin{array}{l}0.0 \\
35\end{array}$ \\
\hline \multicolumn{28}{|c|}{ Lesotho workshop } \\
\hline 1 & 0.416 & $\begin{array}{l}0.4 \\
58\end{array}$ & $\begin{array}{l}0.1 \\
26\end{array}$ & $\begin{array}{l}0.0 \\
08\end{array}$ & $\begin{array}{l}0.6 \\
87\end{array}$ & $\begin{array}{l}0.0 \\
69\end{array}$ & $\begin{array}{l}0.2 \\
44\end{array}$ & $\begin{array}{l}0.1 \\
07\end{array}$ & \begin{tabular}{|l}
0.64 \\
1
\end{tabular} & $\begin{array}{l}0.29 \\
3\end{array}$ & $\begin{array}{l}0 . \\
67\end{array}$ & $\begin{array}{l}0 . \\
86\end{array}$ & & & $\begin{array}{l}0.1 \\
53\end{array}$ & $\begin{array}{l}0.0 \\
70\end{array}$ & $\begin{array}{l}0.0 \\
90\end{array}$ & $\begin{array}{l}0.8 \\
89\end{array}$ & $\begin{array}{l}0.1 \\
11\end{array}$ & $\begin{array}{l}0.0 \\
00\end{array}$ & $\begin{array}{l}0.8 \\
57\end{array}$ & $\begin{array}{l}0.1 \\
43\end{array}$ & $\begin{array}{l}0.0 \\
00\end{array}$ & $\begin{array}{l}0.8 \\
33\end{array}$ & $\begin{array}{l}0.1 \\
67\end{array}$ & $\begin{array}{l}0.0 \\
00\end{array}$ & $\begin{array}{l}0.0 \\
42\end{array}$ \\
\hline 2 & 0.413 & 0.3 & 0.2 & 0.0 & 0.6 & 0.1 & 0.1 & 0.0 & 0.65 & 0.29 & 0. & 0 . & & & 0.1 & 0.0 & 0.0 & 0.8 & 0.1 & 0.0 & 0.8 & 0.1 & 0.0 & 0.8 & 0.1 & 0.0 & 0.0 \\
\hline
\end{tabular}




\begin{tabular}{|c|c|c|c|c|c|c|c|c|c|c|c|c|c|c|c|c|c|c|c|c|c|c|c|c|c|c|}
\hline \multirow{2}{*}{$\begin{array}{l}\text { Pa } \\
\text { rt. } \\
\text { no. }\end{array}$} & \multicolumn{4}{|c|}{ Level 2.1} & \multicolumn{4}{|c|}{ Level 2.2} & \multicolumn{4}{|c|}{ Level 3.1} & \multicolumn{4}{|c|}{ Level 3.2} & \multicolumn{3}{|c|}{ Level 3.3} & \multicolumn{3}{|c|}{ Level 3.4} & \multicolumn{3}{|c|}{ Level 3.5} & \multirow{2}{*}{$\begin{array}{l}\text { Av } \\
\text { g. } \\
\text { C } \\
\text { R }\end{array}$} \\
\hline & $\begin{array}{l}\text { Tech. } \\
\text { /Eq. }\end{array}$ & $\begin{array}{l}\text { Inf } \\
\text { ra. }\end{array}$ & $\begin{array}{l}\text { Pr } \\
\text { oc. }\end{array}$ & $\begin{array}{l}\mathbf{C} \\
\mathbf{R}\end{array}$ & $\begin{array}{l}\mathbf{E q} \\
\cdot\end{array}$ & $\begin{array}{l}\text { Tr } \\
\text { an. }\end{array}$ & $\begin{array}{l}\text { Co } \\
\text { nt. }\end{array}$ & $\begin{array}{l}\mathbf{C} \\
\mathbf{R}\end{array}$ & $\begin{array}{l}\text { SA } \\
\text { SSI } \\
-E\end{array}$ & $\begin{array}{l}\text { SA } \\
\text { SSI } \\
-\mathbf{M}\end{array}$ & $\begin{array}{l}\mathbf{O} \\
\mathbf{A} \\
\mathbf{B}\end{array}$ & $\begin{array}{l}\mathbf{C} \\
\mathbf{R}\end{array}$ & $\begin{array}{l}\mathbf{E} \\
\mathbf{W} \\
\mathbf{T}\end{array}$ & $\begin{array}{l}\mathbf{G} \\
\mathbf{T}\end{array}$ & IT & $\begin{array}{l}\mathbf{C} \\
\mathbf{R}\end{array}$ & $\begin{array}{l}\mathbf{E} \\
\mathbf{C}\end{array}$ & $\begin{array}{l}\mathbf{n E} \\
\mathrm{C}\end{array}$ & $\begin{array}{l}\mathbf{C} \\
\mathbf{R}\end{array}$ & ES & $\begin{array}{l}\mathbf{n E} \\
\mathrm{S}\end{array}$ & $\begin{array}{l}\mathbf{C} \\
\mathbf{R}\end{array}$ & $\begin{array}{l}\mathbf{D} \\
\mathbf{P}\end{array}$ & $\mathbf{n P}$ & $\begin{array}{l}\mathbf{C} \\
\mathbf{R}\end{array}$ & \\
\hline & & 27 & 60 & 46 & 59 & 85 & 56 & 25 & 5 & 0 & 55 & 69 & 85 & 49 & 66 & 69 & 75 & 25 & 00 & 75 & 25 & 00 & 75 & 25 & 00 & 30 \\
\hline 3 & 0.413 & $\begin{array}{l}0.3 \\
27\end{array}$ & $\begin{array}{l}0.2 \\
60\end{array}$ & $\begin{array}{l}0.0 \\
46\end{array}$ & $\begin{array}{l}0.5 \\
50\end{array}$ & $\begin{array}{l}0.2 \\
10\end{array}$ & $\begin{array}{l}0.2 \\
40\end{array}$ & $\begin{array}{l}0.0 \\
16\end{array}$ & $\begin{array}{l}0.65 \\
5\end{array}$ & $\begin{array}{l}0.29 \\
0\end{array}$ & $\begin{array}{l}0.0 \\
55\end{array}$ & $\begin{array}{l}0.0 \\
69\end{array}$ & $\begin{array}{l}0.7 \\
93\end{array}$ & $\begin{array}{l}0.1 \\
31\end{array}$ & $\begin{array}{l}0.0 \\
76\end{array}$ & $\begin{array}{l}0.0 \\
19\end{array}$ & $\begin{array}{l}0.9 \\
00\end{array}$ & $\begin{array}{l}0.1 \\
00\end{array}$ & $\begin{array}{l}0.0 \\
00\end{array}$ & $\begin{array}{l}0.8 \\
75\end{array}$ & $\begin{array}{l}0.1 \\
25\end{array}$ & $\begin{array}{l}0.0 \\
00\end{array}$ & $\begin{array}{l}0.8 \\
75\end{array}$ & $\begin{array}{l}0.1 \\
25\end{array}$ & $\begin{array}{l}0.0 \\
00\end{array}$ & $\begin{array}{l}0.0 \\
21\end{array}$ \\
\hline 4 & 0.333 & $\begin{array}{l}0.3 \\
33\end{array}$ & $\begin{array}{l}0.3 \\
33\end{array}$ & $\begin{array}{l}0.0 \\
00\end{array}$ & $\begin{array}{l}0.2 \\
50\end{array}$ & $\begin{array}{l}0.2 \\
50\end{array}$ & $\begin{array}{l}0.5 \\
00\end{array}$ & $\begin{array}{l}0.0 \\
00\end{array}$ & $\begin{array}{l}0.71 \\
7\end{array}$ & $\begin{array}{l}0.21 \\
7\end{array}$ & $\begin{array}{l}0.0 \\
66\end{array}$ & $\begin{array}{l}0.0 \\
32\end{array}$ & $\begin{array}{l}0.7 \\
70\end{array}$ & $\begin{array}{l}0.1 \\
62\end{array}$ & $\begin{array}{l}0.1 \\
68\end{array}$ & $\begin{array}{l}0.0 \\
46\end{array}$ & $\begin{array}{l}0.9 \\
00\end{array}$ & $\begin{array}{l}0.1 \\
00\end{array}$ & $\begin{array}{l}0.0 \\
00\end{array}$ & $\begin{array}{l}0.9 \\
00\end{array}$ & $\begin{array}{l}0.1 \\
00\end{array}$ & $\begin{array}{l}0.0 \\
00\end{array}$ & $\begin{array}{l}0.9 \\
00\end{array}$ & $\begin{array}{l}0.1 \\
00\end{array}$ & $\begin{array}{l}0.0 \\
00\end{array}$ & $\begin{array}{l}0.0 \\
11\end{array}$ \\
\hline 5 & 0.333 & $\begin{array}{l}0.3 \\
33\end{array}$ & $\begin{array}{l}0.3 \\
33\end{array}$ & $\begin{array}{l}0.0 \\
00\end{array}$ & $\begin{array}{l}0.2 \\
50\end{array}$ & $\begin{array}{l}0.2 \\
50\end{array}$ & $\begin{array}{l}0.5 \\
00\end{array}$ & $\begin{array}{l}0.0 \\
00\end{array}$ & $\begin{array}{l}0.73 \\
5\end{array}$ & $\begin{array}{l}0.20 \\
7\end{array}$ & $\begin{array}{l}0.0 \\
58\end{array}$ & $\begin{array}{l}0.1 \\
01\end{array}$ & $\begin{array}{l}0.7 \\
70\end{array}$ & $\begin{array}{l}0.1 \\
62\end{array}$ & $\begin{array}{l}0.1 \\
68\end{array}$ & $\begin{array}{l}0.0 \\
46\end{array}$ & $\begin{array}{l}0.9 \\
00\end{array}$ & $\begin{array}{l}0.1 \\
00\end{array}$ & $\begin{array}{l}0.0 \\
00\end{array}$ & $\begin{array}{l}0.9 \\
00\end{array}$ & $\begin{array}{l}0.1 \\
00\end{array}$ & $\begin{array}{l}0.0 \\
00\end{array}$ & $\begin{array}{l}0.9 \\
00\end{array}$ & $\begin{array}{l}0.1 \\
00\end{array}$ & $\begin{array}{l}0.0 \\
00\end{array}$ & $\begin{array}{l}0.0 \\
21\end{array}$ \\
\hline 6 & 0.413 & $\begin{array}{l}0.3 \\
27\end{array}$ & $\begin{array}{l}0.2 \\
60\end{array}$ & $\begin{array}{l}0.0 \\
46\end{array}$ & $\begin{array}{l}0.5 \\
15\end{array}$ & $\begin{array}{l}0.0 \\
97\end{array}$ & $\begin{array}{l}0.3 \\
88\end{array}$ & $\begin{array}{l}0.0 \\
69\end{array}$ & $\begin{array}{l}0.60 \\
6\end{array}$ & $\begin{array}{l}0.33 \\
3\end{array}$ & $\begin{array}{l}0.0 \\
61\end{array}$ & $\begin{array}{l}0.0 \\
08\end{array}$ & $\begin{array}{l}0.7 \\
77\end{array}$ & $\begin{array}{l}0.1 \\
53\end{array}$ & $\begin{array}{l}0.0 \\
70\end{array}$ & $\begin{array}{l}0.0 \\
90\end{array}$ & $\begin{array}{l}0.9 \\
00\end{array}$ & $\begin{array}{l}0.1 \\
00\end{array}$ & $\begin{array}{l}0.0 \\
00\end{array}$ & $\begin{array}{l}0.9 \\
00\end{array}$ & $\begin{array}{l}0.1 \\
00\end{array}$ & $\begin{array}{l}0.0 \\
00\end{array}$ & $\begin{array}{l}0.9 \\
00\end{array}$ & $\begin{array}{l}0.1 \\
00\end{array}$ & $\begin{array}{l}0.0 \\
00\end{array}$ & $\begin{array}{l}0.0 \\
30\end{array}$ \\
\hline 7 & 0.333 & $\begin{array}{l}0.3 \\
33\end{array}$ & $\begin{array}{l}0.3 \\
33\end{array}$ & $\begin{array}{l}0.0 \\
00\end{array}$ & $\begin{array}{l}0.4 \\
29\end{array}$ & $\begin{array}{l}0.1 \\
43\end{array}$ & $\begin{array}{l}0.4 \\
29\end{array}$ & $\begin{array}{l}0.0 \\
00\end{array}$ & $\begin{array}{l}0.73 \\
5\end{array}$ & $\begin{array}{l}0.20 \\
7\end{array}$ & $\begin{array}{l}0.0 \\
58\end{array}$ & $\begin{array}{l}0.1 \\
01\end{array}$ & $\begin{array}{l}0.7 \\
73\end{array}$ & $\begin{array}{l}0.1 \\
39\end{array}$ & $\begin{array}{l}0.0 \\
88\end{array}$ & $\begin{array}{l}0.0 \\
46\end{array}$ & $\begin{array}{l}0.8 \\
75\end{array}$ & $\begin{array}{l}0.1 \\
25\end{array}$ & $\begin{array}{l}0.0 \\
00\end{array}$ & $\begin{array}{l}0.8 \\
75\end{array}$ & $\begin{array}{l}0.1 \\
25\end{array}$ & $\begin{array}{l}0.0 \\
00\end{array}$ & $\begin{array}{l}0.8 \\
75\end{array}$ & $\begin{array}{l}0.1 \\
25\end{array}$ & $\begin{array}{l}0.0 \\
00\end{array}$ & $\begin{array}{l}0.0 \\
21\end{array}$ \\
\hline 8 & 0.413 & $\begin{array}{l}0.3 \\
27\end{array}$ & $\begin{array}{l}0.2 \\
60\end{array}$ & $\begin{array}{l}0.0 \\
46\end{array}$ & $\begin{array}{l}0.7 \\
85\end{array}$ & $\begin{array}{l}0.1 \\
49\end{array}$ & $\begin{array}{l}0.0 \\
66\end{array}$ & $\begin{array}{l}0.0 \\
46\end{array}$ & $\begin{array}{l}0.76 \\
3\end{array}$ & $\begin{array}{l}0.17 \\
6\end{array}$ & $\begin{array}{l}0.0 \\
61\end{array}$ & $\begin{array}{l}0.0 \\
93\end{array}$ & $\begin{array}{l}0.6 \\
96\end{array}$ & $\begin{array}{l}0.2 \\
29\end{array}$ & $\begin{array}{l}0.0 \\
75\end{array}$ & $\begin{array}{l}0.0 \\
66\end{array}$ & $\begin{array}{l}0.8 \\
89\end{array}$ & $\begin{array}{l}0.1 \\
11\end{array}$ & $\begin{array}{l}0.0 \\
00\end{array}$ & $\begin{array}{l}0.8 \\
89\end{array}$ & $\begin{array}{l}0.1 \\
11\end{array}$ & $\begin{array}{l}0.0 \\
00\end{array}$ & $\begin{array}{l}0.8 \\
89\end{array}$ & $\begin{array}{l}0.1 \\
11\end{array}$ & $\begin{array}{l}0.0 \\
00\end{array}$ & $\begin{array}{l}0.0 \\
36\end{array}$ \\
\hline 9 & 0.444 & $\begin{array}{l}0.4 \\
44\end{array}$ & $\begin{array}{l}0.1 \\
11\end{array}$ & $\begin{array}{l}0.0 \\
00\end{array}$ & $\begin{array}{l}0.3 \\
33\end{array}$ & $\begin{array}{l}0.3 \\
33\end{array}$ & $\begin{array}{l}0.3 \\
33\end{array}$ & $\begin{array}{l}0.0 \\
00\end{array}$ & $\begin{array}{l}0.73 \\
3\end{array}$ & $\begin{array}{l}0.19 \\
9\end{array}$ & $\begin{array}{l}0.0 \\
68\end{array}$ & $\begin{array}{l}0.0 \\
81\end{array}$ & $\begin{array}{l}0.7 \\
61\end{array}$ & $\begin{array}{l}0.1 \\
66\end{array}$ & $\begin{array}{l}0.0 \\
73\end{array}$ & $\begin{array}{l}0.0 \\
63\end{array}$ & $\begin{array}{l}0.9 \\
00\end{array}$ & $\begin{array}{l}0.1 \\
00\end{array}$ & $\begin{array}{l}0.0 \\
00\end{array}$ & $\begin{array}{l}0.9 \\
00\end{array}$ & $\begin{array}{l}0.1 \\
00\end{array}$ & $\begin{array}{l}0.0 \\
00\end{array}$ & $\begin{array}{l}0.9 \\
00\end{array}$ & $\begin{array}{l}0.1 \\
00\end{array}$ & $\begin{array}{l}0.0 \\
00\end{array}$ & $\begin{array}{l}0.0 \\
21\end{array}$ \\
\hline 10 & 0.462 & $\begin{array}{l}0.4 \\
62\end{array}$ & $\begin{array}{l}0.0 \\
77\end{array}$ & $\begin{array}{l}0.0 \\
00\end{array}$ & $\begin{array}{l}0.7 \\
14\end{array}$ & $\begin{array}{l}0.1 \\
43\end{array}$ & $\begin{array}{l}0.1 \\
43\end{array}$ & $\begin{array}{l}0.0 \\
00\end{array}$ & $\begin{array}{l}0.64 \\
4\end{array}$ & $\begin{array}{l}0.27 \\
1\end{array}$ & $\begin{array}{l}0.0 \\
85\end{array}$ & $\begin{array}{l}0.0 \\
46\end{array}$ & $\begin{array}{l}0.6 \\
99\end{array}$ & $\begin{array}{l}0.2 \\
37\end{array}$ & $\begin{array}{l}0.0 \\
64\end{array}$ & $\begin{array}{l}0.0 \\
81\end{array}$ & $\begin{array}{l}0.8 \\
33\end{array}$ & $\begin{array}{l}0.1 \\
67\end{array}$ & $\begin{array}{l}0.0 \\
00\end{array}$ & $\begin{array}{l}0.7 \\
50\end{array}$ & $\begin{array}{l}0.2 \\
50\end{array}$ & $\begin{array}{l}0.0 \\
00\end{array}$ & $\begin{array}{l}0.8 \\
75\end{array}$ & $\begin{array}{l}0.1 \\
25\end{array}$ & $\begin{array}{l}0.0 \\
00\end{array}$ & $\begin{array}{l}0.0 \\
18\end{array}$ \\
\hline 11 & 0.773 & $\begin{array}{l}0.0 \\
93\end{array}$ & $\begin{array}{l}0.1 \\
34\end{array}$ & $\begin{array}{l}0.0 \\
93\end{array}$ & $\begin{array}{l}0.4 \\
82\end{array}$ & $\begin{array}{l}0.0 \\
91\end{array}$ & $\begin{array}{l}0.4 \\
27\end{array}$ & $\begin{array}{l}0.0 \\
13\end{array}$ & $\begin{array}{l}0.64 \\
8\end{array}$ & $\begin{array}{l}0.30 \\
0\end{array}$ & $\begin{array}{l}0.0 \\
52\end{array}$ & $\begin{array}{l}0.0 \\
93\end{array}$ & $\begin{array}{l}0.6 \\
55\end{array}$ & $\begin{array}{l}0.2 \\
90\end{array}$ & $\begin{array}{l}0.0 \\
55\end{array}$ & $\begin{array}{l}0.0 \\
69\end{array}$ & $\begin{array}{l}0.8 \\
75\end{array}$ & $\begin{array}{l}0.1 \\
25\end{array}$ & $\begin{array}{l}0.0 \\
00\end{array}$ & $\begin{array}{l}0.8 \\
89\end{array}$ & $\begin{array}{l}0.1 \\
11\end{array}$ & $\begin{array}{l}0.0 \\
00\end{array}$ & $\begin{array}{l}0.9 \\
00\end{array}$ & $\begin{array}{l}0.1 \\
00\end{array}$ & $\begin{array}{l}0.0 \\
00\end{array}$ & $\begin{array}{l}0.0 \\
38\end{array}$ \\
\hline
\end{tabular}




\begin{tabular}{|c|c|c|c|c|c|c|c|c|c|c|c|c|c|c|c|c|c|c|c|c|c|c|c|c|c|c|}
\hline \multirow{2}{*}{$\begin{array}{l}\text { Pa } \\
\text { rt. } \\
\text { no. }\end{array}$} & \multicolumn{4}{|c|}{ Level 2.1} & \multicolumn{4}{|c|}{ Level 2.2} & \multicolumn{4}{|c|}{ Level 3.1} & \multicolumn{4}{|c|}{ Level 3.2} & \multicolumn{3}{|c|}{ Level 3.3} & \multicolumn{3}{|c|}{ Level 3.4} & \multicolumn{3}{|c|}{ Level 3.5} & \multirow{2}{*}{$\begin{array}{l}\text { Av } \\
\text { g. } \\
\text { C } \\
\text { R }\end{array}$} \\
\hline & $\begin{array}{l}\text { Tech. } \\
\text { /Eq. }\end{array}$ & $\begin{array}{l}\text { Inf } \\
\text { ra. }\end{array}$ & $\begin{array}{l}\text { Pr } \\
\text { oc. }\end{array}$ & $\begin{array}{l}\mathbf{C} \\
\mathbf{R}\end{array}$ & $\begin{array}{l}\mathbf{E q} \\
.\end{array}$ & $\begin{array}{l}\text { Tr } \\
\text { an. }\end{array}$ & $\begin{array}{l}\text { Co } \\
\text { nt. }\end{array}$ & $\begin{array}{l}\mathbf{C} \\
\mathbf{R}\end{array}$ & $\begin{array}{l}\text { SA } \\
\text { SSI } \\
-E\end{array}$ & $\begin{array}{l}\text { SA } \\
\text { SSI } \\
-\mathbf{M}\end{array}$ & $\begin{array}{l}\mathbf{O} \\
\mathbf{A} \\
\mathbf{B}\end{array}$ & $\begin{array}{l}\mathbf{C} \\
\mathbf{R}\end{array}$ & $\begin{array}{l}\mathbf{E} \\
\mathbf{W} \\
\mathbf{T}\end{array}$ & $\begin{array}{l}\mathbf{G} \\
\mathbf{T}\end{array}$ & IT & $\begin{array}{l}\mathbf{C} \\
\mathbf{R}\end{array}$ & $\begin{array}{l}\mathbf{E} \\
\mathbf{C}\end{array}$ & $\begin{array}{l}\mathbf{n E} \\
\mathbf{C}\end{array}$ & $\begin{array}{l}\mathbf{C} \\
\mathbf{R}\end{array}$ & ES & $\begin{array}{l}\mathbf{n E} \\
\mathrm{S}\end{array}$ & $\begin{array}{l}\mathbf{C} \\
\mathbf{R}\end{array}$ & $\begin{array}{l}\mathbf{D} \\
\mathbf{P}\end{array}$ & $\mathbf{n P}$ & $\begin{array}{l}\mathbf{C} \\
\mathbf{R}\end{array}$ & \\
\hline $\begin{array}{l}\mathrm{M} \\
\text { ea } \\
\mathrm{n}\end{array}$ & 0.420 & $\begin{array}{l}0.3 \\
21\end{array}$ & $\begin{array}{l}0.2 \\
03\end{array}$ & - & $\begin{array}{l}0.4 \\
80\end{array}$ & $\begin{array}{l}0.1 \\
58\end{array}$ & $\begin{array}{l}0.2 \\
68\end{array}$ & - & $\begin{array}{l}0.68 \\
3\end{array}$ & $\begin{array}{l}0.24 \\
8\end{array}$ & $\begin{array}{l}0.0 \\
62\end{array}$ & - & $\begin{array}{l}0.7 \\
49\end{array}$ & $\begin{array}{l}0.1 \\
74\end{array}$ & $\begin{array}{l}0.0 \\
82\end{array}$ & - & $\begin{array}{l}0.8 \\
85\end{array}$ & $\begin{array}{l}0.1 \\
13\end{array}$ & - & $\begin{array}{l}0.8 \\
73\end{array}$ & $\begin{array}{l}0.1 \\
22\end{array}$ & - & $\begin{array}{l}0.8 \\
84\end{array}$ & $\begin{array}{l}0.1 \\
15\end{array}$ & - & - \\
\hline $\begin{array}{l}\text { Av } \\
\text { g. }\end{array}$ & 0.431 & $\begin{array}{l}0.3 \\
42\end{array}$ & $\begin{array}{l}0.2 \\
26\end{array}$ & $\begin{array}{l}0.0 \\
26\end{array}$ & $\begin{array}{l}0.5 \\
14\end{array}$ & $\begin{array}{l}0.1 \\
75\end{array}$ & $\begin{array}{l}0.3 \\
11\end{array}$ & $\begin{array}{l}0.0 \\
25\end{array}$ & $\begin{array}{l}0.68 \\
5\end{array}$ & $\begin{array}{l}0.25 \\
3\end{array}$ & $\begin{array}{l}0.0 \\
62\end{array}$ & $\begin{array}{l}0.0 \\
71\end{array}$ & $\begin{array}{l}0.7 \\
51\end{array}$ & $\begin{array}{l}0.1 \\
79\end{array}$ & $\begin{array}{l}0.0 \\
88\end{array}$ & $\begin{array}{l}0.0 \\
62\end{array}$ & $\begin{array}{l}0.8 \\
85\end{array}$ & $\begin{array}{l}0.1 \\
15\end{array}$ & $\begin{array}{l}0.0 \\
00\end{array}$ & $\begin{array}{l}0.8 \\
74\end{array}$ & $\begin{array}{l}0.1 \\
26\end{array}$ & $\begin{array}{l}0.0 \\
00\end{array}$ & $\begin{array}{l}0.8 \\
84\end{array}$ & $\begin{array}{l}0.1 \\
16\end{array}$ & $\begin{array}{l}0.0 \\
00\end{array}$ & $\begin{array}{l}0.0 \\
50\end{array}$ \\
\hline $\begin{array}{l}\text { St } \\
\text { d. } \\
\text { de } \\
\text { v. }\end{array}$ & 0.122 & $\begin{array}{l}0.1 \\
01\end{array}$ & $\begin{array}{l}0.0 \\
96\end{array}$ & $\begin{array}{l}0.0 \\
31\end{array}$ & $\begin{array}{l}0.1 \\
86\end{array}$ & $\begin{array}{l}0.0 \\
80\end{array}$ & $\begin{array}{l}0.1 \\
51\end{array}$ & $\begin{array}{l}0.0 \\
35\end{array}$ & $\begin{array}{l}0.05 \\
2\end{array}$ & $\begin{array}{l}0.05 \\
3\end{array}$ & $\begin{array}{l}0.0 \\
09\end{array}$ & $\begin{array}{l}0.0 \\
30\end{array}$ & $\begin{array}{l}0.0 \\
45\end{array}$ & $\begin{array}{l}0.0 \\
50\end{array}$ & $\begin{array}{l}0.0 \\
40\end{array}$ & $\begin{array}{l}0.0 \\
22\end{array}$ & $\begin{array}{l}0.0 \\
20\end{array}$ & $\begin{array}{l}0.0 \\
20\end{array}$ & $\begin{array}{l}0.0 \\
00\end{array}$ & $\begin{array}{l}0.0 \\
43\end{array}$ & $\begin{array}{l}0.0 \\
43\end{array}$ & $\begin{array}{l}0.0 \\
00\end{array}$ & $\begin{array}{l}0.0 \\
21\end{array}$ & $\begin{array}{l}0.0 \\
21\end{array}$ & $\begin{array}{l}0.0 \\
00\end{array}$ & $\begin{array}{l}0.0 \\
62\end{array}$ \\
\hline
\end{tabular}


Table 7.

AHP results for the waste disposal life cycle phase (onsite)

\begin{tabular}{|c|c|c|c|c|c|c|c|c|c|c|c|c|c|c|c|c|c|c|c|c|c|c|}
\hline \multirow{2}{*}{$\begin{array}{l}\text { Par } \\
\text { t. } \\
\text { no. }\end{array}$} & \multicolumn{3}{|c|}{ Level 2.1} & \multicolumn{4}{|c|}{ Level 2.2} & \multicolumn{4}{|c|}{ Level 3.1} & \multicolumn{4}{|c|}{ Level 3.2} & \multicolumn{3}{|c|}{ Level 3.3} & \multicolumn{3}{|c|}{ Level 3.4} & \multirow{2}{*}{$\begin{array}{c}\text { Avg } \\
\dot{C} \\
\text { CR } \\
\end{array}$} \\
\hline & $\begin{array}{l}\text { Tech./ } \\
\text { Eq. }\end{array}$ & $\begin{array}{l}\text { Pro } \\
\text { c. }\end{array}$ & CR & Eq. & $\begin{array}{l}\text { Tra } \\
\text { n. }\end{array}$ & $\begin{array}{l}\text { Con } \\
\text { t. }\end{array}$ & CR & OD & CI & El & CR & $\begin{array}{l}\text { EW } \\
\text { T }\end{array}$ & GT & IT & CR & EC & $\begin{array}{l}\mathbf{n E} \\
\mathbf{C}\end{array}$ & CR & DF & $\mathbf{n P}$ & CR & \\
\hline \multicolumn{23}{|c|}{ South African workshop } \\
\hline 1 & 0.500 & $\begin{array}{l}0.5 \\
00\end{array}$ & $\begin{array}{l}0.0 \\
00\end{array}$ & $\begin{array}{l}0.4 \\
89\end{array}$ & $\begin{array}{l}0.06 \\
7\end{array}$ & $\begin{array}{l}0.44 \\
4\end{array}$ & $\begin{array}{l}0.0 \\
08\end{array}$ & $\begin{array}{l}0.0 \\
55\end{array}$ & $\begin{array}{l}0.2 \\
03\end{array}$ & $\begin{array}{l}0 . \\
42\end{array}$ & \begin{tabular}{|l|}
0.3 \\
76
\end{tabular} & $\begin{array}{l}0.81 \\
4\end{array}$ & $\begin{array}{l}0.1 \\
14\end{array}$ & $\begin{array}{l}0 . \\
72\end{array}$ & $\begin{array}{l}0.0 \\
46\end{array}$ & $\begin{array}{l}0.9 \\
00\end{array}$ & $\begin{array}{l}0.1 \\
00\end{array}$ & $\begin{array}{l}0.0 \\
00\end{array}$ & $\begin{array}{l}0.9 \\
00\end{array}$ & \begin{tabular}{|l}
0.1 \\
00
\end{tabular} & $\begin{array}{l}0.0 \\
00\end{array}$ & $\begin{array}{l}0.0 \\
72\end{array}$ \\
\hline 2 & 0.500 & $\begin{array}{l}0.5 \\
00\end{array}$ & $\begin{array}{l}0.0 \\
00\end{array}$ & $\begin{array}{l}0.5 \\
40\end{array}$ & $\begin{array}{l}0.16 \\
3\end{array}$ & $\begin{array}{l}0.29 \\
7\end{array}$ & $\begin{array}{l}0.0 \\
08\end{array}$ & $\begin{array}{l}0.0 \\
58\end{array}$ & $\begin{array}{l}0 . \\
51\end{array}$ & $\begin{array}{l}0 . \\
91\end{array}$ & \begin{tabular}{|l|}
0.1 \\
56
\end{tabular} & $\begin{array}{l}0.78 \\
0\end{array}$ & $\begin{array}{l}0.1 \\
70\end{array}$ & $\begin{array}{l}0 . \\
50\end{array}$ & $\begin{array}{l}0.2 \\
75\end{array}$ & $\begin{array}{l}0.9 \\
00\end{array}$ & $\begin{array}{l}0.1 \\
00\end{array}$ & $\begin{array}{l}0.0 \\
00\end{array}$ & $\begin{array}{l}0.8 \\
75\end{array}$ & $\begin{array}{l}0.1 \\
25\end{array}$ & $\begin{array}{l}0.0 \\
00\end{array}$ & $\begin{array}{l}0.0 \\
73\end{array}$ \\
\hline 3 & 0.500 & $\begin{array}{l}0.5 \\
00\end{array}$ & $\begin{array}{l}0.0 \\
00\end{array}$ & $\begin{array}{l}0.3 \\
33\end{array}$ & $\begin{array}{l}0.33 \\
3\end{array}$ & $\begin{array}{l}0.33 \\
3\end{array}$ & $\begin{array}{l}0.0 \\
00\end{array}$ & $\begin{array}{l}0.0 \\
67\end{array}$ & $\begin{array}{l}0.2 \\
72\end{array}$ & $\begin{array}{l}0 . \\
38\end{array}$ & $\begin{array}{l}0.0 \\
38\end{array}$ & $\begin{array}{l}0.75 \\
7\end{array}$ & $\begin{array}{l}0.1 \\
88\end{array}$ & $\begin{array}{l}0 . \\
54\end{array}$ & $\begin{array}{l}0.2 \\
70\end{array}$ & $\begin{array}{l}0.8 \\
75\end{array}$ & $\begin{array}{l}0.1 \\
25\end{array}$ & $\begin{array}{l}0.0 \\
00\end{array}$ & $\begin{array}{l}0.8 \\
00\end{array}$ & \begin{tabular}{|l}
0.2 \\
00
\end{tabular} & $\begin{array}{l}0.0 \\
00\end{array}$ & $\begin{array}{l}0.0 \\
51\end{array}$ \\
\hline 4 & 0.667 & $\begin{array}{l}0.3 \\
33\end{array}$ & $\begin{array}{l}0.0 \\
00\end{array}$ & $\begin{array}{l}0.2 \\
68\end{array}$ & $\begin{array}{l}0.11 \\
7\end{array}$ & $\begin{array}{l}0.61 \\
4\end{array}$ & $\begin{array}{l}0.0 \\
63\end{array}$ & $\begin{array}{l}0.0 \\
75\end{array}$ & $\begin{array}{l}0 . \\
33\end{array}$ & $\begin{array}{l}0 . \\
92\end{array}$ & $\begin{array}{l}0.0 \\
12\end{array}$ & $\begin{array}{l}0.77 \\
2\end{array}$ & $\begin{array}{l}0.1 \\
73\end{array}$ & $\begin{array}{l}0 . \\
55\end{array}$ & $\begin{array}{l}0.1 \\
80\end{array}$ & $\begin{array}{l}0.8 \\
75\end{array}$ & $\begin{array}{l}0.1 \\
25\end{array}$ & $\begin{array}{l}0.0 \\
00\end{array}$ & $\begin{array}{l}0.8 \\
57\end{array}$ & $\begin{array}{l}0.1 \\
43\end{array}$ & $\begin{array}{l}0.0 \\
00\end{array}$ & $\begin{array}{l}0.0 \\
43\end{array}$ \\
\hline 5 & 0.200 & $\begin{array}{l}0.8 \\
00\end{array}$ & $\begin{array}{l}0.0 \\
00\end{array}$ & $\begin{array}{l}0.3 \\
33\end{array}$ & $\begin{array}{l}0.33 \\
3\end{array}$ & $\begin{array}{l}0.33 \\
3\end{array}$ & $\begin{array}{l}0.0 \\
00\end{array}$ & $\begin{array}{l}0.0 \\
50\end{array}$ & $\begin{array}{l}0 . \\
70\end{array}$ & $\begin{array}{l}0 . \\
80\end{array}$ & $\begin{array}{l}0.2 \\
75\end{array}$ & $\begin{array}{l}0.79 \\
7\end{array}$ & $\begin{array}{l}0.1 \\
51\end{array}$ & $\begin{array}{l}0 . \\
52\end{array}$ & $\begin{array}{l}0.2 \\
54\end{array}$ & $\begin{array}{l}0.9 \\
00\end{array}$ & $\begin{array}{l}0.1 \\
00\end{array}$ & $\begin{array}{l}0.0 \\
00\end{array}$ & $\begin{array}{l}0.9 \\
00\end{array}$ & \begin{tabular}{|l}
0.1 \\
00
\end{tabular} & $\begin{array}{l}0.0 \\
00\end{array}$ & $\begin{array}{l}0.0 \\
88\end{array}$ \\
\hline 6 & 0.500 & $\begin{array}{l}0.5 \\
00\end{array}$ & $\begin{array}{l}0.0 \\
00\end{array}$ & $\begin{array}{l}0.3 \\
33\end{array}$ & $\begin{array}{l}0.33 \\
3\end{array}$ & $\begin{array}{l}0.33 \\
3\end{array}$ & $\begin{array}{l}0.0 \\
00\end{array}$ & $\begin{array}{l}0.1 \\
14\end{array}$ & $\begin{array}{l}0 . \\
00\end{array}$ & $\begin{array}{l}0 . \\
86\end{array}$ & $\begin{array}{l}0.0 \\
16\end{array}$ & $\begin{array}{l}0.80 \\
9\end{array}$ & $\begin{array}{l}0.0 \\
94\end{array}$ & $\begin{array}{l}0 . \\
97\end{array}$ & $\begin{array}{l}0.0 \\
01\end{array}$ & $\begin{array}{l}0.9 \\
00\end{array}$ & $\begin{array}{l}0.1 \\
00\end{array}$ & $\begin{array}{l}0.0 \\
00\end{array}$ & $\begin{array}{l}0.9 \\
00\end{array}$ & $\begin{array}{l}0.1 \\
00\end{array}$ & $\begin{array}{l}0.0 \\
00\end{array}$ & $\begin{array}{l}0.0 \\
03\end{array}$ \\
\hline 7 & 0.500 & $\begin{array}{l}0.5 \\
00\end{array}$ & $\begin{array}{l}0.0 \\
00\end{array}$ & $\begin{array}{l}0.3 \\
33\end{array}$ & $\begin{array}{l}0.33 \\
3\end{array}$ & $\begin{array}{l}0.33 \\
3\end{array}$ & $\begin{array}{l}0.0 \\
00\end{array}$ & $\begin{array}{l}0.0 \\
47\end{array}$ & $\begin{array}{l}0 . \\
78\end{array}$ & 0. & $\begin{array}{l}0.3 \\
30\end{array}$ & $\begin{array}{l}0.77 \\
5\end{array}$ & $\begin{array}{l}0.1 \\
78\end{array}$ & $\begin{array}{l}0 . \\
47\end{array}$ & $\begin{array}{l}0.3 \\
30\end{array}$ & $\begin{array}{l}0.9 \\
00\end{array}$ & $\begin{array}{l}0.1 \\
00\end{array}$ & $\begin{array}{l}0.0 \\
00\end{array}$ & $\begin{array}{l}0.8 \\
89\end{array}$ & $\begin{array}{l}0.1 \\
11\end{array}$ & $\begin{array}{l}0.0 \\
00\end{array}$ & $\begin{array}{l}0.1 \\
10\end{array}$ \\
\hline 8 & 0.500 & $\begin{array}{l}0.5 \\
00\end{array}$ & $\begin{array}{l}0.0 \\
00\end{array}$ & $\begin{array}{l}0.3 \\
33\end{array}$ & $\begin{array}{l}0.33 \\
3\end{array}$ & $\begin{array}{l}0.33 \\
3\end{array}$ & $\begin{array}{l}0.0 \\
00\end{array}$ & $\begin{array}{l}0.0 \\
54\end{array}$ & $\begin{array}{l}0.2 \\
33\end{array}$ & $\begin{array}{l}0 . \\
12\end{array}$ & $\begin{array}{l}0.2 \\
13\end{array}$ & $\begin{array}{l}0.80 \\
0\end{array}$ & $\begin{array}{l}0.1 \\
00\end{array}$ & $\begin{array}{l}0 . \\
00\end{array}$ & $\begin{array}{l}0.0 \\
00\end{array}$ & $\begin{array}{l}0.8 \\
89\end{array}$ & $\begin{array}{l}0.1 \\
11\end{array}$ & $\begin{array}{l}0.0 \\
00\end{array}$ & $\begin{array}{l}0.9 \\
00\end{array}$ & $\begin{array}{l}0.1 \\
00\end{array}$ & $\begin{array}{l}0.0 \\
00\end{array}$ & $\begin{array}{l}0.0 \\
36\end{array}$ \\
\hline 9 & 0.500 & 0.5 & 0.0 & 0.6 & 0.10 & 0.25 & 0.0 & 0.0 & 0. & 0. & 0.0 & 0.58 & 0.3 & 0.1 & 0.0 & 0.8 & 0.1 & 0.0 & 0.8 & 0.1 & 0.0 & 0.0 \\
\hline
\end{tabular}




\begin{tabular}{|c|c|c|c|c|c|c|c|c|c|c|c|c|c|c|c|c|c|c|c|c|c|c|}
\hline \multirow{2}{*}{$\begin{array}{l}\text { Par } \\
\text { t. } \\
\text { no. }\end{array}$} & \multicolumn{3}{|c|}{ Level 2.1} & \multicolumn{4}{|c|}{ Level 2.2} & \multicolumn{4}{|c|}{ Level 3.1} & \multicolumn{4}{|c|}{ Level 3.2} & \multicolumn{3}{|c|}{ Level 3.3} & \multicolumn{3}{|c|}{ Level 3.4} & \multirow{2}{*}{$\begin{array}{c}\text { Avg } \\
\dot{C R} \\
\end{array}$} \\
\hline & $\begin{array}{l}\text { Tech./ } \\
\text { Eq. }\end{array}$ & $\begin{array}{l}\text { Pro } \\
\text { c. }\end{array}$ & $\mathbf{C R}$ & Eq. & $\begin{array}{l}\text { Tra } \\
\text { n. }\end{array}$ & $\begin{array}{l}\text { Con } \\
\text { t. }\end{array}$ & CR & OD & CD & EP & $\mathbf{C R}$ & $\begin{array}{l}\mathbf{E W} \\
\mathbf{T}\end{array}$ & GT & IT & CR & EC & $\begin{array}{l}\mathbf{n E} \\
\mathbf{C}\end{array}$ & $\mathbf{C R}$ & DP & $\mathbf{n P}$ & CR & \\
\hline & & 00 & 00 & 37 & 5 & 8 & 33 & 78 & 71 & 50 & 86 & 8 & 23 & 89 & 08 & 33 & 67 & 00 & 57 & 43 & 00 & 21 \\
\hline 10 & 0.500 & $\begin{array}{l}0.5 \\
00\end{array}$ & $\begin{array}{l}0.0 \\
00\end{array}$ & $\begin{array}{l}0.4 \\
55\end{array}$ & $\begin{array}{l}0.09 \\
1\end{array}$ & $\begin{array}{l}0.45 \\
5\end{array}$ & $\begin{array}{l}0.0 \\
00\end{array}$ & $\begin{array}{l}0.0 \\
63\end{array}$ & $\begin{array}{l}0.2 \\
65\end{array}$ & $\begin{array}{l}0.6 \\
72\end{array}$ & $\begin{array}{l}0.0 \\
25\end{array}$ & $\begin{array}{l}0.66 \\
9\end{array}$ & $\begin{array}{l}0.2 \\
43\end{array}$ & $\begin{array}{l}0.0 \\
88\end{array}$ & $\begin{array}{l}0.0 \\
06\end{array}$ & $\begin{array}{l}0.8 \\
75\end{array}$ & $\begin{array}{l}0.1 \\
25\end{array}$ & $\begin{array}{l}0.0 \\
00\end{array}$ & $\begin{array}{l}0.7 \\
50\end{array}$ & $\begin{array}{l}0.2 \\
50\end{array}$ & $\begin{array}{l}0.0 \\
00\end{array}$ & $\begin{array}{l}0.0 \\
05\end{array}$ \\
\hline 11 & 0.833 & $\begin{array}{l}0.1 \\
67\end{array}$ & $\begin{array}{l}0.0 \\
00\end{array}$ & $\begin{array}{l}0.3 \\
33\end{array}$ & $\begin{array}{l}0.33 \\
3\end{array}$ & $\begin{array}{l}0.33 \\
3\end{array}$ & $\begin{array}{l}0.0 \\
00\end{array}$ & $\begin{array}{l}0.0 \\
46\end{array}$ & $\begin{array}{l}0.1 \\
67\end{array}$ & $\begin{array}{l}0.7 \\
87\end{array}$ & $\begin{array}{l}0.3 \\
76\end{array}$ & $\begin{array}{l}0.72 \\
2\end{array}$ & $\begin{array}{l}0.2 \\
27\end{array}$ & $\begin{array}{l}0.0 \\
51\end{array}$ & $\begin{array}{l}0.1 \\
80\end{array}$ & $\begin{array}{l}0.9 \\
00\end{array}$ & $\begin{array}{l}0.1 \\
00\end{array}$ & $\begin{array}{l}0.0 \\
00\end{array}$ & $\begin{array}{l}0.7 \\
50\end{array}$ & $\begin{array}{l}0.2 \\
50\end{array}$ & $\begin{array}{l}0.0 \\
00\end{array}$ & $\begin{array}{l}0.0 \\
93\end{array}$ \\
\hline $\begin{array}{l}\mathrm{Me} \\
\text { an }\end{array}$ & 0.495 & $\begin{array}{l}0.4 \\
55\end{array}$ & - & $\begin{array}{l}0.3 \\
86\end{array}$ & $\begin{array}{l}0.19 \\
6\end{array}$ & $\begin{array}{l}0.35 \\
9\end{array}$ & - & $\begin{array}{l}0.0 \\
78\end{array}$ & $\begin{array}{l}0.2 \\
10\end{array}$ & $\begin{array}{l}0.3 \\
89\end{array}$ & - & $\begin{array}{l}0.75 \\
0\end{array}$ & $\begin{array}{l}0.1 \\
67\end{array}$ & $\begin{array}{l}0.0 \\
66\end{array}$ & - & $\begin{array}{l}0.8 \\
86\end{array}$ & $\begin{array}{l}0.1 \\
12\end{array}$ & - & $\begin{array}{l}0.8 \\
51\end{array}$ & $\begin{array}{l}0.1 \\
38\end{array}$ & - & - \\
\hline Avg & 0.518 & $\begin{array}{l}0.4 \\
82\end{array}$ & $\begin{array}{l}0.0 \\
00\end{array}$ & $\begin{array}{l}0.3 \\
99\end{array}$ & $\begin{array}{l}0.23 \\
1\end{array}$ & $\begin{array}{l}0.37 \\
0\end{array}$ & $\begin{array}{l}0.0 \\
10\end{array}$ & $\begin{array}{l}0.0 \\
79\end{array}$ & $\begin{array}{l}0.2 \\
28\end{array}$ & $\begin{array}{l}0.5 \\
68\end{array}$ & $\begin{array}{l}0.0 \\
35\end{array}$ & $\begin{array}{l}0.75 \\
3\end{array}$ & $\begin{array}{l}0.1 \\
78\end{array}$ & $\begin{array}{l}0.0 \\
69\end{array}$ & $\begin{array}{l}0.1 \\
41\end{array}$ & $\begin{array}{l}0.8 \\
86\end{array}$ & $\begin{array}{l}0.1 \\
14\end{array}$ & $\begin{array}{l}0.0 \\
00\end{array}$ & $\begin{array}{l}0.8 \\
53\end{array}$ & $\begin{array}{l}0.1 \\
47\end{array}$ & $\begin{array}{l}0.0 \\
00\end{array}$ & $\begin{array}{l}0.0 \\
54\end{array}$ \\
\hline $\begin{array}{l}\text { Std. } \\
\text { dev. }\end{array}$ & 0.150 & $\begin{array}{l}0.1 \\
50\end{array}$ & $\begin{array}{l}0.0 \\
00\end{array}$ & $\begin{array}{l}0.1 \\
14\end{array}$ & $\begin{array}{l}0.11 \\
9\end{array}$ & $\begin{array}{l}0.09 \\
9\end{array}$ & $\begin{array}{l}0.0 \\
20\end{array}$ & $\begin{array}{l}0.0 \\
20\end{array}$ & $\begin{array}{l}0.0 \\
92\end{array}$ & $\begin{array}{l}0.3 \\
05\end{array}$ & $\begin{array}{l}0.0 \\
30\end{array}$ & $\begin{array}{l}0.06 \\
9\end{array}$ & $\begin{array}{l}0.0 \\
68\end{array}$ & $\begin{array}{l}0.0 \\
21\end{array}$ & $\begin{array}{l}0.1 \\
31\end{array}$ & $\begin{array}{l}0.0 \\
21\end{array}$ & $\begin{array}{l}0.0 \\
21\end{array}$ & $\begin{array}{l}0.0 \\
00\end{array}$ & $\begin{array}{l}0.0 \\
59\end{array}$ & $\begin{array}{l}0.0 \\
59\end{array}$ & $\begin{array}{l}0.0 \\
00\end{array}$ & $\begin{array}{l}0.0 \\
36\end{array}$ \\
\hline \multicolumn{18}{|c|}{ Lesotho workshop } & & & & & \\
\hline & \multicolumn{3}{|c|}{ Level 2.1} & \multicolumn{3}{|c|}{ Level 2.2} & \multicolumn{4}{|c|}{ Level 3.1} & \multicolumn{3}{|c|}{ Level 3.2} & \multicolumn{3}{|c|}{ Level 3.3} & \multirow{2}{*}{$\begin{array}{l}\text { Avg } \\
\text { CR }\end{array}$} & & & & & \\
\hline & $\begin{array}{l}\text { Tech./E } \\
\text { q. }\end{array}$ & $\begin{array}{l}\text { Pro } \\
\text { c. }\end{array}$ & CR & Eq. & $\begin{array}{l}\text { Con } \\
\text { t. }\end{array}$ & $\mathrm{CR}$ & OD & $\mathrm{CD}$ & EP & CR & $\mathrm{EC}$ & $\mathrm{nEC}$ & CR & DP & $\mathrm{nP}$ & CR & & & & & & \\
\hline 1 & 0.500 & $\begin{array}{l}0.5 \\
00\end{array}$ & $\begin{array}{l}0.0 \\
00\end{array}$ & $\begin{array}{l}0.5 \\
00\end{array}$ & $\begin{array}{l}0.50 \\
0\end{array}$ & $\begin{array}{l}0.00 \\
0\end{array}$ & $\begin{array}{l}0.0 \\
67\end{array}$ & $\begin{array}{l}0.2 \\
93\end{array}$ & $\begin{array}{l}0.6 \\
41\end{array}$ & $\begin{array}{l}0.0 \\
86\end{array}$ & $\begin{array}{l}0.8 \\
89\end{array}$ & $\begin{array}{l}0.11 \\
1\end{array}$ & $\begin{array}{l}0.0 \\
00\end{array}$ & $\begin{array}{l}0.8 \\
75\end{array}$ & $\begin{array}{l}0.1 \\
25\end{array}$ & $\begin{array}{l}0.0 \\
00\end{array}$ & $\begin{array}{l}0.0 \\
17\end{array}$ & & & & & \\
\hline
\end{tabular}




\begin{tabular}{|c|c|c|c|c|c|c|c|c|c|c|c|c|c|c|c|c|c|c|c|c|c|c|}
\hline \multirow{2}{*}{$\begin{array}{l}\text { Par } \\
\text { t. } \\
\text { no. }\end{array}$} & \multicolumn{3}{|c|}{ Level 2.1} & \multicolumn{4}{|c|}{ Level 2.2} & \multicolumn{4}{|c|}{ Level 3.1} & \multicolumn{4}{|c|}{ Level 3.2} & \multicolumn{3}{|c|}{ Level 3.3} & \multicolumn{3}{|c|}{ Level 3.4} & \multirow{2}{*}{$\begin{array}{c}\text { Avg } \\
\dot{C R} \\
\end{array}$} \\
\hline & $\begin{array}{l}\text { Tech./ } \\
\text { Eq. }\end{array}$ & $\begin{array}{l}\text { Pro } \\
\text { c. }\end{array}$ & CR & Eq. & $\begin{array}{l}\text { Tra } \\
\text { n. }\end{array}$ & $\begin{array}{l}\text { Con } \\
\text { t. }\end{array}$ & CR & OD & CD & EP & CR & $\begin{array}{l}\mathbf{E W} \\
\mathbf{T}\end{array}$ & GT & IT & CR & EC & $\begin{array}{l}\mathbf{n E} \\
\mathbf{C}\end{array}$ & CR & DP & $\mathbf{n P}$ & CR & \\
\hline 2 & 0.500 & $\begin{array}{l}0.5 \\
00\end{array}$ & $\begin{array}{l}0.0 \\
00\end{array}$ & $\begin{array}{l}0.8 \\
75\end{array}$ & $\begin{array}{l}0.12 \\
5\end{array}$ & $\begin{array}{l}0.00 \\
0\end{array}$ & $\begin{array}{l}0.0 \\
72\end{array}$ & $\begin{array}{l}0.2 \\
79\end{array}$ & $\begin{array}{l}0.6 \\
41\end{array}$ & $\begin{array}{l}0.0 \\
86\end{array}$ & $\begin{array}{l}0.8 \\
75\end{array}$ & $\begin{array}{l}0.12 \\
5\end{array}$ & $\begin{array}{l}0.0 \\
00\end{array}$ & $\begin{array}{l}0.9 \\
00\end{array}$ & $\begin{array}{l}0.1 \\
00\end{array}$ & $\begin{array}{l}0.0 \\
00\end{array}$ & $\begin{array}{l}0.0 \\
17\end{array}$ & & & & & \\
\hline 3 & 0.500 & $\begin{array}{l}0.5 \\
00\end{array}$ & $\begin{array}{l}0.0 \\
00\end{array}$ & $\begin{array}{l}0.8 \\
75\end{array}$ & $\begin{array}{l}0.12 \\
5\end{array}$ & $\begin{array}{l}0.00 \\
0\end{array}$ & $\begin{array}{l}0.0 \\
75\end{array}$ & $\begin{array}{l}0.3 \\
33\end{array}$ & $\begin{array}{l}0.5 \\
92\end{array}$ & $\begin{array}{l}0.0 \\
12\end{array}$ & $\begin{array}{l}0.8 \\
75\end{array}$ & $\begin{array}{l}0.12 \\
5\end{array}$ & $\begin{array}{l}0.0 \\
00\end{array}$ & $\begin{array}{l}0.9 \\
00\end{array}$ & $\begin{array}{l}0.1 \\
00\end{array}$ & $\begin{array}{l}0.0 \\
00\end{array}$ & $\begin{array}{l}0.0 \\
02\end{array}$ & & & & & \\
\hline 4 & 0.500 & $\begin{array}{l}0.5 \\
00\end{array}$ & $\begin{array}{l}0.0 \\
00\end{array}$ & $\begin{array}{l}0.3 \\
33\end{array}$ & $\begin{array}{l}0.66 \\
7\end{array}$ & $\begin{array}{l}0.00 \\
0\end{array}$ & $\begin{array}{l}0.0 \\
70\end{array}$ & $\begin{array}{l}0.1 \\
78\end{array}$ & $\begin{array}{l}0.7 \\
51\end{array}$ & $\begin{array}{l}0.0 \\
25\end{array}$ & $\begin{array}{l}0.9 \\
00\end{array}$ & $\begin{array}{l}0.10 \\
0\end{array}$ & $\begin{array}{l}0.0 \\
00\end{array}$ & $\begin{array}{l}0.9 \\
00\end{array}$ & $\begin{array}{l}0.1 \\
00\end{array}$ & $\begin{array}{l}0.0 \\
00\end{array}$ & $\begin{array}{l}0.0 \\
05\end{array}$ & & & & & \\
\hline 5 & 0.500 & $\begin{array}{l}0.5 \\
00\end{array}$ & $\begin{array}{l}0.0 \\
00\end{array}$ & $\begin{array}{l}0.3 \\
33\end{array}$ & $\begin{array}{l}0.66 \\
7\end{array}$ & $\begin{array}{l}0.00 \\
0\end{array}$ & $\begin{array}{l}0.0 \\
70\end{array}$ & $\begin{array}{l}0.1 \\
78\end{array}$ & $\begin{array}{l}0.7 \\
51\end{array}$ & $\begin{array}{l}0.0 \\
25\end{array}$ & $\begin{array}{l}0.9 \\
00\end{array}$ & $\begin{array}{l}0.10 \\
0\end{array}$ & $\begin{array}{l}0.0 \\
00\end{array}$ & $\begin{array}{l}0.9 \\
00\end{array}$ & $\begin{array}{l}0.1 \\
00\end{array}$ & $\begin{array}{l}0.0 \\
00\end{array}$ & $\begin{array}{l}0.0 \\
05\end{array}$ & & & & & \\
\hline 6 & 0.500 & $\begin{array}{l}0.5 \\
00\end{array}$ & $\begin{array}{l}0.0 \\
00\end{array}$ & $\begin{array}{l}0.5 \\
00\end{array}$ & $\begin{array}{l}0.50 \\
0\end{array}$ & $\begin{array}{l}0.00 \\
0\end{array}$ & $\begin{array}{l}0.1 \\
43\end{array}$ & $\begin{array}{l}0.1 \\
43\end{array}$ & $\begin{array}{l}0.7 \\
14\end{array}$ & $\begin{array}{l}0.0 \\
00\end{array}$ & $\begin{array}{l}0.9 \\
00\end{array}$ & $\begin{array}{l}0.10 \\
0\end{array}$ & $\begin{array}{l}0.0 \\
00\end{array}$ & $\begin{array}{l}0.9 \\
00\end{array}$ & $\begin{array}{l}0.1 \\
00\end{array}$ & $\begin{array}{l}0.0 \\
00\end{array}$ & $\begin{array}{l}0.0 \\
00\end{array}$ & & & & & \\
\hline 7 & 0.500 & $\begin{array}{l}0.5 \\
00\end{array}$ & $\begin{array}{l}0.0 \\
00\end{array}$ & $\begin{array}{l}0.5 \\
00\end{array}$ & $\begin{array}{l}0.50 \\
0\end{array}$ & $\begin{array}{l}0.00 \\
0\end{array}$ & $\begin{array}{l}0.1 \\
25\end{array}$ & $\begin{array}{l}0.0 \\
79\end{array}$ & $\begin{array}{l}0.7 \\
96\end{array}$ & $\begin{array}{l}0.0 \\
46\end{array}$ & $\begin{array}{l}0.8 \\
89\end{array}$ & $\begin{array}{l}0.11 \\
1\end{array}$ & $\begin{array}{l}0.0 \\
00\end{array}$ & $\begin{array}{l}0.9 \\
00\end{array}$ & $\begin{array}{l}0.1 \\
00\end{array}$ & $\begin{array}{l}0.0 \\
00\end{array}$ & $\begin{array}{l}0.0 \\
09\end{array}$ & & & & & \\
\hline 8 & 0.500 & $\begin{array}{l}0.5 \\
00\end{array}$ & $\begin{array}{l}0.0 \\
00\end{array}$ & $\begin{array}{l}0.8 \\
00\end{array}$ & $\begin{array}{l}0.20 \\
0\end{array}$ & $\begin{array}{l}0.00 \\
0\end{array}$ & $\begin{array}{l}0.1 \\
25\end{array}$ & $\begin{array}{l}0.0 \\
79\end{array}$ & $\begin{array}{l}0.7 \\
96\end{array}$ & $\begin{array}{l}0.0 \\
46\end{array}$ & $\begin{array}{l}0.8 \\
57\end{array}$ & $\begin{array}{l}0.14 \\
3\end{array}$ & $\begin{array}{l}0.0 \\
00\end{array}$ & $\begin{array}{l}0.8 \\
75\end{array}$ & $\begin{array}{l}0.1 \\
25\end{array}$ & $\begin{array}{l}0.0 \\
00\end{array}$ & $\begin{array}{l}0.0 \\
09\end{array}$ & & & & & \\
\hline 9 & 0.500 & $\begin{array}{l}0.5 \\
00\end{array}$ & $\begin{array}{l}0.0 \\
00\end{array}$ & $\begin{array}{l}0.5 \\
00\end{array}$ & $\begin{array}{l}0.50 \\
0\end{array}$ & $\begin{array}{l}0.00 \\
0\end{array}$ & $\begin{array}{l}0.0 \\
61\end{array}$ & $\begin{array}{l}0.1 \\
76\end{array}$ & $\begin{array}{l}0.7 \\
63\end{array}$ & $\begin{array}{l}0.0 \\
93\end{array}$ & $\begin{array}{l}0.9 \\
00\end{array}$ & $\begin{array}{l}0.10 \\
0\end{array}$ & $\begin{array}{l}0.0 \\
00\end{array}$ & $\begin{array}{l}0.9 \\
00\end{array}$ & $\begin{array}{l}0.1 \\
00\end{array}$ & $\begin{array}{l}0.0 \\
00\end{array}$ & $\begin{array}{l}0.0 \\
19\end{array}$ & & & & & \\
\hline 10 & 0.500 & $\begin{array}{l}0.5 \\
00\end{array}$ & $\begin{array}{l}0.0 \\
00\end{array}$ & $\begin{array}{l}0.8 \\
75\end{array}$ & $\begin{array}{l}0.12 \\
5\end{array}$ & $\begin{array}{l}0.00 \\
0\end{array}$ & $\begin{array}{l}0.0 \\
58\end{array}$ & $\begin{array}{l}0.2 \\
07\end{array}$ & $\begin{array}{l}0.7 \\
35\end{array}$ & $\begin{array}{l}0.1 \\
01\end{array}$ & $\begin{array}{l}0.8 \\
75\end{array}$ & $\begin{array}{l}0.12 \\
5\end{array}$ & $\begin{array}{l}0.0 \\
00\end{array}$ & $\begin{array}{l}0.9 \\
00\end{array}$ & $\begin{array}{l}0.1 \\
00\end{array}$ & $\begin{array}{l}0.0 \\
00\end{array}$ & $\begin{array}{l}0.0 \\
20\end{array}$ & & & & & \\
\hline 11 & 0.833 & $\begin{array}{l}0.1 \\
67\end{array}$ & $\begin{array}{l}0.0 \\
00\end{array}$ & $\begin{array}{l}0.8 \\
00\end{array}$ & $\begin{array}{l}0.20 \\
0\end{array}$ & $\begin{array}{l}0.00 \\
0\end{array}$ & $\begin{array}{l}0.0 \\
56\end{array}$ & $\begin{array}{l}0.2 \\
43\end{array}$ & $\begin{array}{l}0.7 \\
01\end{array}$ & $\begin{array}{l}0.0 \\
93\end{array}$ & $\begin{array}{l}0.8 \\
33\end{array}$ & $\begin{array}{l}0.16 \\
7\end{array}$ & $\begin{array}{l}0.0 \\
00\end{array}$ & $\begin{array}{l}0.7 \\
50\end{array}$ & $\begin{array}{l}0.2 \\
50\end{array}$ & $\begin{array}{l}0.0 \\
00\end{array}$ & $\begin{array}{l}0.0 \\
19\end{array}$ & & & & & \\
\hline
\end{tabular}




\begin{tabular}{|c|c|c|c|c|c|c|c|c|c|c|c|c|c|c|c|c|c|c|c|c|c|c|}
\hline \multirow{2}{*}{$\begin{array}{l}\text { Par } \\
\text { t. } \\
\text { no. }\end{array}$} & \multicolumn{3}{|c|}{ Level 2.1} & \multicolumn{4}{|c|}{ Level 2.2} & \multicolumn{4}{|c|}{ Level 3.1} & \multicolumn{4}{|c|}{ Level 3.2} & \multicolumn{3}{|c|}{ Level 3.3} & \multicolumn{3}{|c|}{ Level 3.4} & \multirow{2}{*}{$\begin{array}{c}\text { Avg } \\
\dot{C R} \\
\end{array}$} \\
\hline & $\begin{array}{l}\text { Tech./ } \\
\text { Eq. }\end{array}$ & $\begin{array}{l}\text { Pro } \\
\text { c. }\end{array}$ & CR & Eq. & $\begin{array}{l}\text { Tra } \\
\text { n. }\end{array}$ & $\begin{array}{l}\text { Con } \\
\text { t. }\end{array}$ & CR & OD & CD & EP & CR & $\begin{array}{l}\mathbf{E W} \\
\mathbf{T}\end{array}$ & GT & IT & CR & EC & $\begin{array}{l}\mathbf{n E} \\
\mathrm{C}\end{array}$ & CR & DP & $\mathbf{n P}$ & CR & \\
\hline $\begin{array}{l}\mathrm{Me} \\
\text { an }\end{array}$ & 0.547 & $\begin{array}{l}0.4 \\
16\end{array}$ & - & $\begin{array}{l}0.5 \\
89\end{array}$ & $\begin{array}{l}0.30 \\
6\end{array}$ & - & $\begin{array}{l}0.0 \\
80\end{array}$ & $\begin{array}{l}0.1 \\
78\end{array}$ & $\begin{array}{l}0.7 \\
16\end{array}$ & - & $\begin{array}{l}0.8 \\
79\end{array}$ & $\begin{array}{l}0.11 \\
9\end{array}$ & - & $\begin{array}{l}0.8 \\
80\end{array}$ & $\begin{array}{l}0.1 \\
14\end{array}$ & - & - & & & & & \\
\hline Avg & 0.558 & $\begin{array}{l}0.4 \\
42\end{array}$ & $\begin{array}{l}0.0 \\
00\end{array}$ & $\begin{array}{l}0.6 \\
26\end{array}$ & $\begin{array}{l}0.37 \\
4\end{array}$ & $\begin{array}{l}0.00 \\
0\end{array}$ & $\begin{array}{l}0.0 \\
84\end{array}$ & $\begin{array}{l}0.1 \\
96\end{array}$ & $\begin{array}{l}0.7 \\
19\end{array}$ & $\begin{array}{l}0.0 \\
55\end{array}$ & $\begin{array}{l}0.8 \\
80\end{array}$ & $\begin{array}{l}0.12 \\
0\end{array}$ & $\begin{array}{l}0.0 \\
00\end{array}$ & $\begin{array}{l}0.8 \\
81\end{array}$ & $\begin{array}{l}0.1 \\
19\end{array}$ & $\begin{array}{l}0.0 \\
00\end{array}$ & $\begin{array}{l}0.0 \\
11\end{array}$ & & & & & \\
\hline $\begin{array}{l}\text { Std. } \\
\text { dev. }\end{array}$ & 0.128 & $\begin{array}{l}0.1 \\
28\end{array}$ & $\begin{array}{l}0.0 \\
00\end{array}$ & $\begin{array}{l}0.2 \\
19\end{array}$ & $\begin{array}{l}0.21 \\
9\end{array}$ & $\begin{array}{l}0.00 \\
0\end{array}$ & $\begin{array}{l}0.0 \\
31\end{array}$ & $\begin{array}{l}0.0 \\
83\end{array}$ & $\begin{array}{l}0.0 \\
68\end{array}$ & $\begin{array}{l}0.0 \\
36\end{array}$ & $\begin{array}{l}0.0 \\
23\end{array}$ & $\begin{array}{l}0.02 \\
3\end{array}$ & $\begin{array}{l}0.0 \\
00\end{array}$ & $\begin{array}{l}0.0 \\
45\end{array}$ & $\begin{array}{l}0.0 \\
45\end{array}$ & $\begin{array}{l}0.0 \\
00\end{array}$ & $\begin{array}{l}0.0 \\
07\end{array}$ & & & & & \\
\hline
\end{tabular}

Table 8.

AHP results for the waste treatment life cycle phase (central)

\begin{tabular}{|c|c|c|c|c|c|c|c|c|c|c|c|c|c|c|c|c|c|c|c|c|c|c|c|c|c|c|c|c|c|c|}
\hline \multirow{2}{*}{$\begin{array}{l}\text { Pa } \\
\text { rt. } \\
\text { no } \\
\cdot\end{array}$} & \multicolumn{4}{|c|}{ Level 2.1} & \multicolumn{4}{|c|}{ Level 2.2} & \multicolumn{8}{|c|}{ Level 3.1} & \multicolumn{4}{|c|}{ Level 3.2} & \multicolumn{3}{|c|}{ Level 3.3} & \multicolumn{3}{|c|}{ Level 3.4} & \multicolumn{3}{|c|}{ Level 3.5} & \multirow{2}{*}{$\begin{array}{l}\text { A } \\
\text { vg } \\
\dot{C} \\
\text { R }\end{array}$} \\
\hline & $\begin{array}{l}\text { Tec } \\
\text { h/E } \\
\text { q. }\end{array}$ & $\begin{array}{l}\text { In } \\
\text { fr } \\
\text { a. }\end{array}$ & $\begin{array}{l}\text { Pr } \\
\text { oc } \\
\text { • }\end{array}$ & $\begin{array}{l}\mathbf{C} \\
\mathbf{R}\end{array}$ & $\begin{array}{l}\mathbf{E} \\
\text { q. }\end{array}$ & $\begin{array}{l}\text { Tr } \\
\text { an } \\
\text {. }\end{array}$ & $\begin{array}{l}\text { C } \\
\text { on } \\
\text { t. }\end{array}$ & $\begin{array}{l}\mathbf{C} \\
\mathbf{R}\end{array}$ & $\begin{array}{l}\mathbf{M} \\
\mathbf{C} \\
\mathbf{I}\end{array}$ & $\begin{array}{l}\text { S } \\
\text { C } \\
\text { I }\end{array}$ & $\begin{array}{l}\text { S } \\
\& \\
D\end{array}$ & $\begin{array}{l}\mathbf{W} \\
\mathbf{S}\end{array}$ & $\begin{array}{l}\text { D } \\
\text { S }\end{array}$ & I & & $\begin{array}{l}\mathbf{C} \\
\mathbf{R}\end{array}$ & $\begin{array}{l}\mathbf{E} \\
\mathbf{W} \\
\mathbf{V}\end{array}$ & $\begin{array}{l}\mathbf{G} \\
\mathbf{V}\end{array}$ & $\begin{array}{l}\text { I } \\
\text { V }\end{array}$ & & $\begin{array}{l}\mathbf{E} \\
\mathrm{C}\end{array}$ & $\begin{array}{l}\mathbf{n} \\
\mathbf{E} \\
\mathbf{C}\end{array}$ & $\begin{array}{l}\mathbf{C} \\
\mathbf{R}\end{array}$ & $\begin{array}{l}\mathbf{E} \\
\mathbf{S}\end{array}$ & $\begin{array}{l}\mathbf{n} \\
\mathbf{E} \\
\mathbf{S}\end{array}$ & $\begin{array}{l}\mathbf{C} \\
\mathbf{R}\end{array}$ & $\begin{array}{l}\mathbf{D} \\
\mathbf{P}\end{array}$ & $\begin{array}{l}\mathbf{n} \\
\mathbf{P}\end{array}$ & $\begin{array}{l}\mathbf{C} \\
\mathbf{R}\end{array}$ & \\
\hline \multicolumn{31}{|c|}{ South African workshop } \\
\hline 1 & $\begin{array}{l}0.57 \\
1\end{array}$ & $\begin{array}{l}0 . \\
14 \\
3\end{array}$ & $\begin{array}{l}0 . \\
28 \\
6\end{array}$ & $\begin{array}{l}0 . \\
43 \\
1\end{array}$ & $\begin{array}{l}0 . \\
47 \\
4\end{array}$ & $\begin{array}{l}0 . \\
05 \\
3\end{array}$ & $\begin{array}{l}0 . \\
47 \\
4\end{array}$ & $\begin{array}{l}0 . \\
00 \\
0\end{array}$ & $\begin{array}{l}0 . \\
54 \\
7\end{array}$ & $\begin{array}{l}0 . \\
06 \\
3\end{array}$ & $\begin{array}{l}0 . \\
04 \\
5\end{array}$ & $\begin{array}{l}0 . \\
14 \\
5\end{array}$ & $\begin{array}{l}0 . \\
09 \\
0\end{array}$ & & & $\begin{array}{l}0 . \\
22 \\
1\end{array}$ & $\begin{array}{l}0 . \\
80 \\
0\end{array}$ & $\begin{array}{l}0 . \\
10 \\
0\end{array}$ & $\begin{array}{l}0 . \\
10 \\
0\end{array}$ & & $\begin{array}{l}0 . \\
90 \\
0\end{array}$ & $\begin{array}{l}0 . \\
10 \\
0\end{array}$ & $\begin{array}{l}0 . \\
00 \\
0\end{array}$ & $\begin{array}{l}0 . \\
90 \\
0\end{array}$ & $\begin{array}{l}0 . \\
10 \\
0\end{array}$ & $\begin{array}{l}0 . \\
00 \\
0\end{array}$ & $\begin{array}{l}0 . \\
90 \\
0\end{array}$ & $\begin{array}{l}0 . \\
10 \\
0\end{array}$ & $\begin{array}{l}0 . \\
00 \\
0\end{array}$ & $\begin{array}{l}0 . \\
10 \\
6\end{array}$ \\
\hline
\end{tabular}




\begin{tabular}{|c|c|c|c|c|c|c|c|c|c|c|c|c|c|c|c|c|c|c|c|c|c|c|c|c|c|c|c|c|c|c|c|c|}
\hline \multirow{2}{*}{$\begin{array}{l}\text { Pa } \\
\text { rt. } \\
\text { no } \\
\text { • }\end{array}$} & \multicolumn{4}{|c|}{ Level 2.1} & \multicolumn{4}{|c|}{ Level 2.2} & \multicolumn{9}{|c|}{ Level 3.1} & \multicolumn{4}{|c|}{ Level 3.2} & \multicolumn{4}{|c|}{ Level 3.3} & \multicolumn{3}{|c|}{ Level 3.4} & \multicolumn{3}{|c|}{ Level 3.5} & \multirow{2}{*}{$\begin{array}{l}\text { A } \\
\text { vg } \\
\dot{C} \\
\text { C } \\
\text { R }\end{array}$} \\
\hline & $\begin{array}{l}\text { Tec } \\
\text { h/E } \\
\text { q. }\end{array}$ & $\begin{array}{l}\text { In } \\
\text { fr } \\
\text { a. }\end{array}$ & $\begin{array}{l}\text { Pr } \\
\text { oc } \\
\text {. }\end{array}$ & $\begin{array}{l}\mathbf{C} \\
\mathbf{R}\end{array}$ & $\begin{array}{l}\text { E } \\
\text { q. }\end{array}$ & $\begin{array}{l}\mathrm{Tr} \\
\text { an } \\
\text {. }\end{array}$ & $\begin{array}{l}\text { C } \\
\text { o } \\
\text { t. }\end{array}$ & & & $\begin{array}{l}\text { M } \\
\mathbf{C} \\
\mathbf{I}\end{array}$ & $\begin{array}{l}\mathbf{S} \\
\mathbf{C} \\
\mathbf{I}\end{array}$ & $\begin{array}{l}\text { S } \\
\& \\
D\end{array}$ & $\begin{array}{l}\text { W } \\
\text { S }\end{array}$ & & & $\mathbf{E}$ & $\begin{array}{l}\mathbf{C} \\
\mathbf{R}\end{array}$ & $\begin{array}{l}\mathbf{E} \\
\mathbf{W} \\
\mathbf{V}\end{array}$ & $\begin{array}{l}\mathbf{G} \\
\mathbf{V}\end{array}$ & 1 & & & $\begin{array}{l}\mathbf{E} \\
\mathbf{C}\end{array}$ & $\begin{array}{l}\mathbf{n} \\
\mathbf{E} \\
\mathbf{C}\end{array}$ & $\begin{array}{l}\mathbf{C} \\
\mathbf{R}\end{array}$ & $\begin{array}{l}\mathbf{E} \\
\mathbf{S}\end{array}$ & $\begin{array}{l}\mathbf{n} \\
\mathbf{E} \\
\mathbf{S}\end{array}$ & $\begin{array}{l}\mathbf{C} \\
\mathbf{R}\end{array}$ & $\begin{array}{l}\mathbf{D} \\
\mathbf{P}\end{array}$ & $\begin{array}{l}\mathbf{n} \\
\mathbf{P}\end{array}$ & $\begin{array}{l}\mathbf{C} \\
\mathbf{R}\end{array}$ & \\
\hline 2 & $\begin{array}{l}0.50 \\
0\end{array}$ & $\begin{array}{l}0 . \\
25 \\
0\end{array}$ & $\begin{array}{l}0 . \\
25 \\
0\end{array}$ & $\begin{array}{l}0 . \\
00 \\
0\end{array}$ & $\begin{array}{l}0 . \\
50 \\
0\end{array}$ & $\begin{array}{l}0 . \\
07 \\
3\end{array}$ & $\begin{array}{l}0 \\
4 \\
7\end{array}$ & 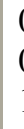 & & $\begin{array}{l}0 . \\
45 \\
1\end{array}$ & $\begin{array}{l}0 . \\
09 \\
6\end{array}$ & $\begin{array}{l}0 . \\
06 \\
9\end{array}$ & $\begin{array}{l}0 \\
3 \\
2\end{array}$ & & & $\begin{array}{l}0 . \\
05 \\
3\end{array}$ & $\begin{array}{l}0 . \\
19 \\
0\end{array}$ & $\begin{array}{l}0 . \\
77 \\
0\end{array}$ & $\begin{array}{l}0 . \\
18 \\
5\end{array}$ & 5 & & & $\begin{array}{l}0 . \\
90 \\
0\end{array}$ & $\begin{array}{l}0 . \\
10 \\
0\end{array}$ & $\begin{array}{l}0 . \\
00 \\
0\end{array}$ & $\begin{array}{l}0 . \\
88 \\
9\end{array}$ & $\begin{array}{l}0 . \\
11 \\
1\end{array}$ & $\begin{array}{l}0 . \\
00 \\
0\end{array}$ & $\begin{array}{l}0 . \\
88 \\
9\end{array}$ & $\begin{array}{l}0 . \\
11 \\
1\end{array}$ & $\begin{array}{l}0 . \\
00 \\
0\end{array}$ & $\begin{array}{l}0 . \\
08 \\
5\end{array}$ \\
\hline 3 & $\begin{array}{l}0.33 \\
3\end{array}$ & $\begin{array}{l}0 . \\
33 \\
3\end{array}$ & $\begin{array}{l}0 . \\
33 \\
3\end{array}$ & $\begin{array}{l}0 . \\
00 \\
0\end{array}$ & $\begin{array}{l}0 . \\
54 \\
0\end{array}$ & $\begin{array}{l}0 . \\
16 \\
3\end{array}$ & $\begin{array}{l}0 \\
2 \\
7\end{array}$ & . & & $\begin{array}{l}0 . \\
50 \\
6\end{array}$ & $\begin{array}{l}0 . \\
05 \\
2\end{array}$ & $\begin{array}{l}0 . \\
16 \\
1\end{array}$ & 6 & & & $\begin{array}{l}0 . \\
03 \\
5\end{array}$ & $\begin{array}{l}0 . \\
17 \\
5\end{array}$ & $\begin{array}{l}0 . \\
77 \\
3\end{array}$ & $\begin{array}{l}0 . \\
13 \\
9\end{array}$ & 0 & ( & & $\begin{array}{l}0 . \\
88 \\
9\end{array}$ & $\begin{array}{l}0 . \\
11 \\
1\end{array}$ & $\begin{array}{l}0 . \\
00 \\
0\end{array}$ & $\begin{array}{l}0 . \\
90 \\
0\end{array}$ & $\begin{array}{l}0 . \\
10 \\
0\end{array}$ & $\begin{array}{l}0 . \\
00 \\
0\end{array}$ & $\begin{array}{l}0 . \\
83 \\
3\end{array}$ & $\begin{array}{l}0 . \\
16 \\
7\end{array}$ & $\begin{array}{l}0 . \\
00 \\
0\end{array}$ & $\begin{array}{l}0 . \\
07 \\
7\end{array}$ \\
\hline 4 & $\begin{array}{l}0.73 \\
2\end{array}$ & $\begin{array}{l}0 . \\
13 \\
0\end{array}$ & $\begin{array}{l}0 . \\
13 \\
8\end{array}$ & $\begin{array}{l}0 . \\
00 \\
3\end{array}$ & $\begin{array}{l}0 . \\
26 \\
0\end{array}$ & $\begin{array}{l}0 . \\
32 \\
7\end{array}$ & $\begin{array}{l}0 \\
4 \\
3\end{array}$ & 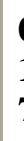 & & $\begin{array}{l}0 . \\
43 \\
4\end{array}$ & $\begin{array}{l}0 . \\
26 \\
4\end{array}$ & $\begin{array}{l}0 . \\
04 \\
6\end{array}$ & $\begin{array}{l}0 \\
1 \\
2\end{array}$ & & & $\begin{array}{l}0 . \\
02 \\
2\end{array}$ & $\begin{array}{l}0 . \\
29 \\
8\end{array}$ & $\begin{array}{l}0 . \\
77 \\
8\end{array}$ & $\begin{array}{l}0 . \\
18 \\
0\end{array}$ & 2 & & & $\begin{array}{l}0 . \\
90 \\
0\end{array}$ & $\begin{array}{l}0 . \\
10 \\
0\end{array}$ & $\begin{array}{l}0 . \\
00 \\
0\end{array}$ & $\begin{array}{l}0 . \\
90 \\
0\end{array}$ & $\begin{array}{l}0 . \\
10 \\
0\end{array}$ & $\begin{array}{l}0 . \\
00 \\
0\end{array}$ & $\begin{array}{l}0 . \\
83 \\
3\end{array}$ & $\begin{array}{l}0 . \\
16 \\
7\end{array}$ & $\begin{array}{l}0 . \\
00 \\
0\end{array}$ & $\begin{array}{l}0 . \\
11 \\
0\end{array}$ \\
\hline 5 & $\begin{array}{l}0.33 \\
3\end{array}$ & $\begin{array}{l}0 . \\
33 \\
3\end{array}$ & $\begin{array}{l}0 . \\
33 \\
3\end{array}$ & $\begin{array}{l}0 . \\
00 \\
0\end{array}$ & $\begin{array}{l}0 . \\
33 \\
3\end{array}$ & $\begin{array}{l}0 . \\
33 \\
3\end{array}$ & $\begin{array}{l}0 \\
3 \\
3\end{array}$ & ( & & $\begin{array}{l}0 . \\
55 \\
5\end{array}$ & $\begin{array}{l}0 . \\
20 \\
0\end{array}$ & $\begin{array}{l}0 . \\
06 \\
8\end{array}$ & $\begin{array}{l}0 \\
4\end{array}$ & & & $\begin{array}{l}0 . \\
02 \\
8\end{array}$ & $\begin{array}{l}0 . \\
10 \\
0\end{array}$ & $\begin{array}{l}0 . \\
77 \\
8\end{array}$ & $\begin{array}{l}0 . \\
18 \\
0\end{array}$ & 0 & & & $\begin{array}{l}0 . \\
90 \\
0\end{array}$ & $\begin{array}{l}0 . \\
10 \\
0\end{array}$ & $\begin{array}{l}0 . \\
00 \\
0\end{array}$ & $\begin{array}{l}0 . \\
87 \\
5\end{array}$ & $\begin{array}{l}0 . \\
12 \\
5\end{array}$ & $\begin{array}{l}0 . \\
00 \\
0\end{array}$ & $\begin{array}{l}0 . \\
90 \\
0\end{array}$ & $\begin{array}{l}0 . \\
10 \\
0\end{array}$ & $\begin{array}{l}0 . \\
00 \\
0\end{array}$ & $\begin{array}{l}0 . \\
08 \\
3\end{array}$ \\
\hline 6 & $\begin{array}{l}0.33 \\
3\end{array}$ & $\begin{array}{l}0 . \\
33 \\
3\end{array}$ & $\begin{array}{l}0 . \\
33 \\
3\end{array}$ & $\begin{array}{l}0 . \\
00 \\
0\end{array}$ & $\begin{array}{l}0 . \\
06 \\
0\end{array}$ & $\begin{array}{l}0 . \\
21 \\
0\end{array}$ & $\begin{array}{l}0 \\
7 \\
0\end{array}$ & 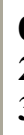 & & $\begin{array}{l}0 . \\
44 \\
4\end{array}$ & $\begin{array}{l}0 . \\
18 \\
9\end{array}$ & $\begin{array}{l}0 . \\
04 \\
9\end{array}$ & 3 & & & $\begin{array}{l}0 . \\
18 \\
7\end{array}$ & $\begin{array}{l}0 . \\
39 \\
7\end{array}$ & $\begin{array}{l}0 . \\
72 \\
4\end{array}$ & $\begin{array}{l}0 . \\
22 \\
8\end{array}$ & $\delta$ & & & $\begin{array}{l}0 . \\
90 \\
0\end{array}$ & $\begin{array}{l}0 . \\
10 \\
0\end{array}$ & $\begin{array}{l}0 . \\
00 \\
0\end{array}$ & $\begin{array}{l}0 . \\
90 \\
0\end{array}$ & $\begin{array}{l}0 . \\
10 \\
0\end{array}$ & $\begin{array}{l}0 . \\
00 \\
0\end{array}$ & $\begin{array}{l}0 . \\
88 \\
9\end{array}$ & $\begin{array}{l}0 . \\
11 \\
1\end{array}$ & $\begin{array}{l}0 . \\
00 \\
0\end{array}$ & $\begin{array}{l}0 . \\
14 \\
6\end{array}$ \\
\hline 7 & $\begin{array}{l}0.16 \\
6\end{array}$ & $\begin{array}{l}0 . \\
22 \\
5\end{array}$ & $\begin{array}{l}0 . \\
61 \\
0\end{array}$ & $\begin{array}{l}0 . \\
08 \\
1\end{array}$ & $\begin{array}{l}0 . \\
55 \\
9\end{array}$ & $\begin{array}{l}0 \\
10 \\
3\end{array}$ & $\begin{array}{l}0 \\
3 \\
9\end{array}$ & & & $\begin{array}{l}0 . \\
36 \\
8\end{array}$ & $\begin{array}{l}0 . \\
37 \\
3\end{array}$ & $\begin{array}{l}0 . \\
08 \\
3\end{array}$ & $\begin{array}{l}0 \\
0 \\
2\end{array}$ & & & $\begin{array}{l}0 . \\
02 \\
4\end{array}$ & $\begin{array}{l}0 . \\
12 \\
9\end{array}$ & $\begin{array}{l}0 . \\
78 \\
3\end{array}$ & $\begin{array}{l}0 \\
17 \\
4\end{array}$ & & & & $\begin{array}{l}0 . \\
90 \\
0\end{array}$ & $\begin{array}{l}0 . \\
10 \\
0\end{array}$ & $\begin{array}{l}0 . \\
00 \\
0\end{array}$ & $\begin{array}{l}0 . \\
90 \\
0\end{array}$ & $\begin{array}{l}0 . \\
10 \\
0\end{array}$ & $\begin{array}{l}0 . \\
00 \\
0\end{array}$ & $\begin{array}{l}0 . \\
80 \\
0\end{array}$ & $\begin{array}{l}0 . \\
20 \\
0\end{array}$ & $\begin{array}{l}0 . \\
00 \\
0\end{array}$ & $\begin{array}{l}0 . \\
12 \\
3\end{array}$ \\
\hline 8 & $\begin{array}{l}0.33 \\
3\end{array}$ & $\begin{array}{l}0 . \\
33 \\
3\end{array}$ & $\begin{array}{l}0 . \\
33 \\
3\end{array}$ & $\begin{array}{l}0 . \\
00 \\
0\end{array}$ & $\begin{array}{l}0 . \\
42 \\
9\end{array}$ & $\begin{array}{l}0 . \\
14 \\
3\end{array}$ & $\begin{array}{l}0 \\
4 \\
9\end{array}$ & ( & & $\begin{array}{l}0 . \\
37 \\
2\end{array}$ & $\begin{array}{l}0 . \\
07 \\
4\end{array}$ & $\begin{array}{l}0 \\
16 \\
3\end{array}$ & 1 & & & $\begin{array}{l}0 . \\
10 \\
8\end{array}$ & $\begin{array}{l}0 . \\
06 \\
1\end{array}$ & $\begin{array}{l}0 . \\
78 \\
4\end{array}$ & $\begin{array}{l}0 \\
13 \\
5\end{array}$ & 0 & 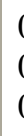 & & $\begin{array}{l}0 . \\
88 \\
9\end{array}$ & $\begin{array}{l}0 . \\
11 \\
1\end{array}$ & $\begin{array}{l}0 . \\
00 \\
0\end{array}$ & $\begin{array}{l}0 . \\
88 \\
9\end{array}$ & $\begin{array}{l}0 . \\
11 \\
1\end{array}$ & $\begin{array}{l}0 . \\
00 \\
0\end{array}$ & $\begin{array}{l}0 . \\
88 \\
9\end{array}$ & $\begin{array}{l}0 . \\
11 \\
1\end{array}$ & $\begin{array}{l}0 . \\
00 \\
0\end{array}$ & $\begin{array}{l}0 . \\
01 \\
3\end{array}$ \\
\hline 9 & $\begin{array}{l}0.23 \\
8\end{array}$ & $\begin{array}{l}0 . \\
13\end{array}$ & $\begin{array}{l}0 . \\
62\end{array}$ & $\begin{array}{l}0 . \\
01\end{array}$ & $\begin{array}{l}0 . \\
19\end{array}$ & $\begin{array}{l}0 . \\
17\end{array}$ & 6 & & & $\begin{array}{l}0 . \\
47\end{array}$ & $\begin{array}{l}0 . \\
22\end{array}$ & $\begin{array}{l}0 . \\
10\end{array}$ & 0 & & & $\begin{array}{l}0 . \\
06\end{array}$ & $\begin{array}{l}0 . \\
03\end{array}$ & $\begin{array}{l}0 . \\
74\end{array}$ & $\begin{array}{l}0 . \\
18\end{array}$ & & & & $\begin{array}{l}0 . \\
90\end{array}$ & $\begin{array}{l}0 . \\
10\end{array}$ & $\begin{array}{l}0 . \\
00\end{array}$ & $\begin{array}{l}0 . \\
87\end{array}$ & $\begin{array}{l}0 . \\
12\end{array}$ & $\begin{array}{l}0 . \\
00\end{array}$ & $\begin{array}{l}0 . \\
85\end{array}$ & $\begin{array}{l}0 . \\
14\end{array}$ & $\begin{array}{l}0 . \\
00\end{array}$ & $\begin{array}{l}0 . \\
01\end{array}$ \\
\hline
\end{tabular}




\begin{tabular}{|c|c|c|c|c|c|c|c|c|c|c|c|c|c|c|c|c|c|c|c|c|c|c|c|c|c|c|c|c|c|}
\hline \multirow{2}{*}{$\begin{array}{l}\text { Pa } \\
\text { rt. } \\
\text { no } \\
\cdot\end{array}$} & \multicolumn{4}{|c|}{ Level 2.1} & \multicolumn{4}{|c|}{ Level 2.2} & \multicolumn{7}{|c|}{ Level 3.1} & \multicolumn{4}{|c|}{ Level 3.2} & \multicolumn{3}{|c|}{ Level 3.3} & \multicolumn{3}{|c|}{ Level 3.4} & \multicolumn{3}{|c|}{ Level 3.5} & \multirow{2}{*}{$\begin{array}{l}\text { A } \\
\text { vg } \\
\dot{C} \\
\text { R }\end{array}$} \\
\hline & \multirow[t]{2}{*}{$\begin{array}{l}\text { Tec } \\
\text { h/E } \\
\text { q. }\end{array}$} & $\begin{array}{l}\text { In } \\
\text { fr } \\
\text { a. }\end{array}$ & $\begin{array}{l}\text { Pr } \\
\text { oc } \\
\cdot\end{array}$ & $\begin{array}{l}\mathbf{C} \\
\mathbf{R}\end{array}$ & $\begin{array}{l}\text { E } \\
\text { q. }\end{array}$ & $\begin{array}{l}\text { Tr } \\
\text { an } \\
.\end{array}$ & $\begin{array}{l}\text { C } \\
\text { on } \\
\text { t. }\end{array}$ & $\begin{array}{l}\mathbf{C} \\
\mathbf{R}\end{array}$ & $\begin{array}{l}\text { M } \\
\text { C } \\
\text { I }\end{array}$ & $\begin{array}{l}\text { S } \\
\text { C } \\
\text { I }\end{array}$ & $\begin{array}{l}\text { S } \\
\& \\
D\end{array}$ & $\begin{array}{l}\text { W } \\
\text { S }\end{array}$ & $\begin{array}{l}\text { D } \\
\text { S }\end{array}$ & $\mathbf{E}$ & $\begin{array}{l}\mathbf{C} \\
\mathbf{R}\end{array}$ & $\begin{array}{l}\mathbf{E} \\
\mathbf{W} \\
\mathbf{V}\end{array}$ & $\begin{array}{l}\mathbf{G} \\
\mathbf{V}\end{array}$ & $\begin{array}{l}\mathbf{I} \\
\mathbf{V}\end{array}$ & $\begin{array}{l}\mathbf{C} \\
\mathbf{R}\end{array}$ & $\begin{array}{l}\mathbf{E} \\
\mathbf{C}\end{array}$ & $\begin{array}{l}\mathbf{n} \\
\mathbf{E} \\
\mathbf{C}\end{array}$ & $\begin{array}{l}\mathbf{C} \\
\mathbf{R}\end{array}$ & $\begin{array}{l}\mathbf{E} \\
\mathbf{S}\end{array}$ & $\begin{array}{l}\mathbf{n} \\
\mathbf{E} \\
\mathbf{S}\end{array}$ & $\begin{array}{l}\mathbf{C} \\
\mathbf{R}\end{array}$ & $\begin{array}{l}\mathbf{D} \\
\mathbf{P}\end{array}$ & $\begin{array}{l}\mathbf{n} \\
\mathbf{P}\end{array}$ & $\begin{array}{l}\mathbf{C} \\
\mathbf{R}\end{array}$ & \\
\hline & & 6 & 5 & 6 & 2 & 4 & 4 & 8 & 4 & 0 & 6 & 9 & 9 & 2 & 6 & 2 & 3 & 5 & 8 & 0 & 0 & 0 & 5 & 5 & 0 & 7 & 3 & 0 & 4 \\
\hline 10 & $\begin{array}{l}0.45 \\
5\end{array}$ & $\begin{array}{l}0 . \\
09 \\
1\end{array}$ & $\begin{array}{l}0 . \\
45 \\
5\end{array}$ & $\begin{array}{l}0 . \\
00 \\
0\end{array}$ & $\begin{array}{l}0 . \\
28 \\
0\end{array}$ & $\begin{array}{l}0 . \\
09 \\
4\end{array}$ & $\begin{array}{l}0 . \\
62 \\
7\end{array}$ & $\begin{array}{l}0 . \\
07 \\
4\end{array}$ & $\begin{array}{l}0 . \\
39 \\
2\end{array}$ & \begin{tabular}{l|}
0. \\
13 \\
2
\end{tabular} & $\begin{array}{l}0 . \\
11 \\
2\end{array}$ & $\begin{array}{l}0 . \\
17 \\
9\end{array}$ & $\begin{array}{l}0 . \\
08 \\
7\end{array}$ & $\begin{array}{l}0 . \\
09 \\
9\end{array}$ & $\begin{array}{l}\mathbf{0 .} \\
33 \\
8\end{array}$ & $\begin{array}{l}0 . \\
77 \\
2\end{array}$ & $\begin{array}{l}0 . \\
17 \\
3\end{array}$ & $\begin{array}{l}0 . \\
05 \\
5\end{array}$ & \begin{tabular}{|l|}
0. \\
18 \\
0
\end{tabular} & $\begin{array}{l}0 . \\
83 \\
3\end{array}$ & $\begin{array}{l}0 . \\
16 \\
7\end{array}$ & $\begin{array}{l}0 . \\
00 \\
0\end{array}$ & $\begin{array}{l}0 . \\
83 \\
3\end{array}$ & $\begin{array}{l}0 . \\
16 \\
7\end{array}$ & $\begin{array}{l}0 . \\
00 \\
0\end{array}$ & $\begin{array}{l}0 . \\
87 \\
5\end{array}$ & $\begin{array}{l}0 . \\
12 \\
5\end{array}$ & $\begin{array}{l}0 . \\
00 \\
0\end{array}$ & $\begin{array}{l}0 . \\
08 \\
5\end{array}$ \\
\hline 11 & $\begin{array}{l}0.05 \\
4\end{array}$ & $\begin{array}{l}0 . \\
21 \\
8\end{array}$ & $\begin{array}{l}0 . \\
72 \\
8\end{array}$ & $\begin{array}{l}0 . \\
14 \\
1\end{array}$ & $\begin{array}{l}0 . \\
05 \\
5\end{array}$ & $\begin{array}{l}0 . \\
17 \\
3\end{array}$ & $\begin{array}{l}0 . \\
77 \\
2\end{array}$ & $\begin{array}{l}\text { 0. } \\
18 \\
0\end{array}$ & $\begin{array}{l}0 . \\
21 \\
3\end{array}$ & \begin{tabular}{l|}
0. \\
09 \\
3
\end{tabular} & $\begin{array}{l}0 . \\
14 \\
1\end{array}$ & $\begin{array}{l}0 . \\
26 \\
1\end{array}$ & $\begin{array}{l}0 . \\
26 \\
1\end{array}$ & $\begin{array}{l}0 . \\
03 \\
2\end{array}$ & $\begin{array}{l}0 . \\
07 \\
6\end{array}$ & $\begin{array}{l}0 . \\
76 \\
3\end{array}$ & $\begin{array}{l}0 . \\
17 \\
8\end{array}$ & $\begin{array}{l}0 . \\
05 \\
8\end{array}$ & $\begin{array}{l}0 . \\
21 \\
3\end{array}$ & $\begin{array}{l}0 . \\
90 \\
0\end{array}$ & $\begin{array}{l}0 . \\
10 \\
0\end{array}$ & $\begin{array}{l}0 . \\
00 \\
0\end{array}$ & $\begin{array}{l}0 . \\
88 \\
9\end{array}$ & $\begin{array}{l}0 . \\
11 \\
1\end{array}$ & $\begin{array}{l}0 . \\
00 \\
0\end{array}$ & $\begin{array}{l}0 . \\
90 \\
0\end{array}$ & $\begin{array}{l}0 . \\
10 \\
0\end{array}$ & $\begin{array}{l}0 . \\
00 \\
0\end{array}$ & $\begin{array}{l}0 . \\
08 \\
7\end{array}$ \\
\hline $\begin{array}{l}\text { M } \\
\text { ea } \\
n\end{array}$ & $\begin{array}{l}0.35 \\
1\end{array}$ & $\begin{array}{l}0 . \\
21 \\
8\end{array}$ & $\begin{array}{l}0 . \\
34 \\
7\end{array}$ & - & $\begin{array}{l}0 . \\
34 \\
9\end{array}$ & $\begin{array}{l}0 . \\
12 \\
0\end{array}$ & $\begin{array}{l}0 . \\
47 \\
5\end{array}$ & - & $\begin{array}{l}0 . \\
38 \\
0\end{array}$ & $\begin{array}{l}0 . \\
13 \\
2\end{array}$ & $\begin{array}{l}0 . \\
11 \\
4\end{array}$ & $\begin{array}{l}0 . \\
12 \\
1\end{array}$ & $\begin{array}{l}0 . \\
11 \\
2\end{array}$ & $\begin{array}{l}0 . \\
04 \\
9\end{array}$ & - & $\begin{array}{l}0 . \\
77 \\
4\end{array}$ & $\begin{array}{l}0 . \\
13 \\
6\end{array}$ & $\begin{array}{l}0 . \\
08 \\
6\end{array}$ & - & $\begin{array}{l}0 . \\
89 \\
2\end{array}$ & $\begin{array}{l}0 . \\
10 \\
7\end{array}$ & - & $\begin{array}{l}0 . \\
88 \\
6\end{array}$ & $\begin{array}{l}0 . \\
11 \\
2\end{array}$ & - & $\begin{array}{l}0 . \\
86 \\
9\end{array}$ & $\begin{array}{l}0 . \\
12 \\
7\end{array}$ & - & - \\
\hline $\begin{array}{l}\text { A } \\
\mathrm{vg}\end{array}$ & $\begin{array}{l}0.38 \\
0\end{array}$ & $\begin{array}{l}0 . \\
24 \\
0\end{array}$ & $\begin{array}{l}0 . \\
37 \\
9\end{array}$ & $\begin{array}{l}0 . \\
01 \\
1\end{array}$ & $\begin{array}{l}0 . \\
36 \\
8\end{array}$ & $\begin{array}{l}0 . \\
14 \\
5\end{array}$ & $\begin{array}{l}0 . \\
48 \\
7\end{array}$ & $\begin{array}{l}0 . \\
01 \\
7\end{array}$ & $\begin{array}{l}0 . \\
40 \\
4\end{array}$ & \begin{tabular}{l|}
0. \\
14 \\
7
\end{tabular} & $\begin{array}{l}0 . \\
12 \\
0\end{array}$ & $\begin{array}{l}0 . \\
14 \\
2\end{array}$ & $\begin{array}{l}0 . \\
13 \\
1\end{array}$ & $\begin{array}{l}0 . \\
05 \\
7\end{array}$ & $\begin{array}{l}0 . \\
06 \\
8\end{array}$ & $\begin{array}{l}0 . \\
77 \\
5\end{array}$ & $\begin{array}{l}0 . \\
13 \\
9\end{array}$ & $\begin{array}{l}0 . \\
08 \\
6\end{array}$ & $\begin{array}{l}0 . \\
05 \\
1\end{array}$ & $\begin{array}{l}0 . \\
89 \\
2\end{array}$ & $\begin{array}{l}0 . \\
10 \\
8\end{array}$ & $\begin{array}{l}0 . \\
00 \\
0\end{array}$ & $\begin{array}{l}0 . \\
88 \\
6\end{array}$ & $\begin{array}{l}0 . \\
11 \\
4\end{array}$ & $\begin{array}{l}0 . \\
00 \\
0\end{array}$ & $\begin{array}{l}0 . \\
87 \\
0\end{array}$ & $\begin{array}{l}0 . \\
13 \\
0\end{array}$ & $\begin{array}{l}0 . \\
00 \\
0\end{array}$ & $\begin{array}{l}0 . \\
08 \\
4\end{array}$ \\
\hline $\begin{array}{l}\text { St } \\
\text { d. } \\
\text { de } \\
\text { v. }\end{array}$ & $\begin{array}{l}0.16 \\
5\end{array}$ & $\begin{array}{l}0 . \\
10 \\
0\end{array}$ & $\begin{array}{l}0 . \\
15 \\
9\end{array}$ & $\begin{array}{l}0 . \\
02 \\
7\end{array}$ & $\begin{array}{l}0 . \\
12 \\
0\end{array}$ & $\begin{array}{l}0 . \\
10 \\
2\end{array}$ & $\begin{array}{l}0 . \\
12 \\
0\end{array}$ & $\begin{array}{l}0 . \\
02 \\
9\end{array}$ & $\begin{array}{l}0 . \\
14 \\
8\end{array}$ & $\begin{array}{l}0 . \\
07 \\
4\end{array}$ & $\begin{array}{l}0 . \\
04 \\
2\end{array}$ & $\begin{array}{l}0 . \\
09 \\
0\end{array}$ & $\begin{array}{l}0 . \\
08 \\
9\end{array}$ & $\begin{array}{l}0 . \\
03 \\
7\end{array}$ & $\begin{array}{l}0 . \\
02 \\
7\end{array}$ & $\begin{array}{l}0 . \\
02 \\
5\end{array}$ & $\begin{array}{l}0 . \\
03 \\
4\end{array}$ & $\begin{array}{l}0 . \\
01 \\
1\end{array}$ & $\begin{array}{l}0 . \\
02 \\
7\end{array}$ & $\begin{array}{l}0 . \\
02 \\
0\end{array}$ & $\begin{array}{l}0 . \\
02 \\
0\end{array}$ & $\begin{array}{l}0 . \\
00 \\
0\end{array}$ & $\begin{array}{l}0 . \\
02 \\
0\end{array}$ & $\begin{array}{l}0 . \\
02 \\
0\end{array}$ & $\begin{array}{l}0 . \\
00 \\
0\end{array}$ & $\begin{array}{l}0 . \\
03 \\
4\end{array}$ & $\begin{array}{l}0 . \\
03 \\
4\end{array}$ & $\begin{array}{l}0 . \\
00 \\
0\end{array}$ & $\begin{array}{l}0 . \\
04 \\
1\end{array}$ \\
\hline Lesc & tho we & KSIIC & & & & & & & & & & & & & & & & & & & & & & & & & & & \\
\hline & Level & & & & Lev & el 2.2 & & & Leve & 3.1 & & Lev & 3.2 & & & Leve & 13.3 & & Lev & 3.4 & & Lev & 3.5 & & A & & & & \\
\hline
\end{tabular}




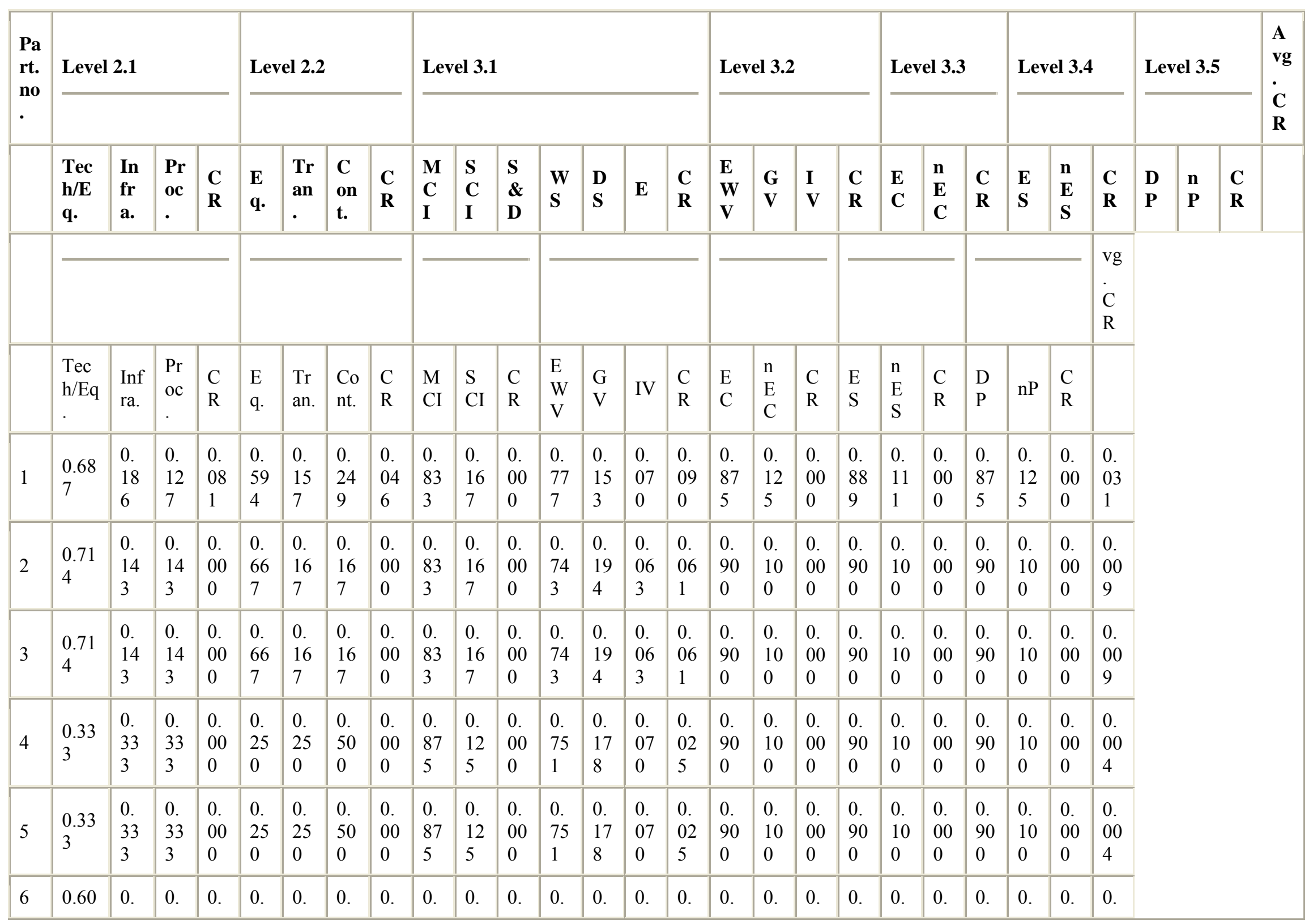




\begin{tabular}{|c|c|c|c|c|c|c|c|c|c|c|c|c|c|c|c|c|c|c|c|c|c|c|c|c|c|c|c|c|c|c|}
\hline \multirow{3}{*}{$\begin{array}{l}\text { Pa } \\
\text { rt. } \\
\text { no } \\
\cdot\end{array}$} & \multicolumn{4}{|c|}{ Level 2.1} & \multicolumn{4}{|c|}{ Level 2.2} & \multicolumn{7}{|c|}{ Level 3.1} & \multicolumn{5}{|c|}{ Level 3.2} & \multicolumn{3}{|c|}{ Level 3.3} & \multicolumn{3}{|c|}{ Level 3.4} & \multicolumn{3}{|c|}{ Level 3.5} & \multirow{2}{*}{$\begin{array}{l}\text { A } \\
\text { vg } \\
\dot{C} \\
\text { R }\end{array}$} \\
\hline & $\begin{array}{l}\text { Tec } \\
\text { h/E } \\
\text { q. } \\
\end{array}$ & $\begin{array}{l}\text { In } \\
\text { fr } \\
\text { a. }\end{array}$ & $\begin{array}{l}\text { Pr } \\
\text { oc } \\
\cdot\end{array}$ & $\begin{array}{l}\mathbf{C} \\
\mathbf{R}\end{array}$ & $\begin{array}{l}\mathbf{E} \\
\mathbf{q} .\end{array}$ & $\begin{array}{l}\text { Tr } \\
\text { an } \\
\cdot \\
\end{array}$ & $\begin{array}{l}\text { C } \\
\text { on } \\
\text { t. }\end{array}$ & $\begin{array}{l}\mathbf{C} \\
\mathbf{R}\end{array}$ & $\begin{array}{l}\mathbf{M} \\
\mathbf{C} \\
\mathbf{I}\end{array}$ & $\begin{array}{l}\text { S } \\
\text { C } \\
\text { I }\end{array}$ & $\begin{array}{l}\text { S } \\
\& \\
\text { D }\end{array}$ & $\begin{array}{l}\text { W } \\
\text { S }\end{array}$ & $\begin{array}{l}\text { D } \\
\text { S }\end{array}$ & I & & & & $\begin{array}{l}\text { G } \\
\text { V }\end{array}$ & $\begin{array}{l}\text { I } \\
\text { V }\end{array}$ & $\begin{array}{l}\mathbf{C} \\
\mathbf{R}\end{array}$ & $\begin{array}{l}\mathbf{E} \\
\mathbf{C}\end{array}$ & $\begin{array}{l}\mathbf{n} \\
\mathbf{E} \\
\mathbf{C}\end{array}$ & $\begin{array}{l}\mathbf{C} \\
\mathbf{R}\end{array}$ & $\begin{array}{l}E \\
S\end{array}$ & E & I & & $\begin{array}{l}\mathbf{n} \\
\mathbf{P}\end{array}$ & $\begin{array}{l}\mathbf{C} \\
\mathbf{R}\end{array}$ & \\
\hline & 0 & $\begin{array}{l}20 \\
0\end{array}$ & $\begin{array}{l}20 \\
0\end{array}$ & $\begin{array}{l}00 \\
0\end{array}$ & $\begin{array}{l}42 \\
9\end{array}$ & $\begin{array}{l}14 \\
3\end{array}$ & $\begin{array}{l}42 \\
9\end{array}$ & $\begin{array}{l}00 \\
0\end{array}$ & $\begin{array}{l}85 \\
7\end{array}$ & $\begin{array}{l}12 \\
5\end{array}$ & $\begin{array}{l}00 \\
0\end{array}$ & $\begin{array}{l}79 \\
9\end{array}$ & $\begin{array}{l}10 \\
5\end{array}$ & 0 & 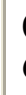 & 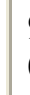 & & $\begin{array}{l}10 \\
0\end{array}$ & $\begin{array}{l}00 \\
0\end{array}$ & $\begin{array}{l}90 \\
0\end{array}$ & $\begin{array}{l}10 \\
0\end{array}$ & $\begin{array}{l}00 \\
0\end{array}$ & $\begin{array}{l}90 \\
0\end{array}$ & $\begin{array}{l}10 \\
0\end{array}$ & $\begin{array}{l}0 \\
0\end{array}$ & 1 & & & & \\
\hline 7 & $\begin{array}{l}0.33 \\
3\end{array}$ & $\begin{array}{l}0 . \\
33 \\
3\end{array}$ & $\begin{array}{l}0 . \\
33 \\
3\end{array}$ & \begin{tabular}{|l}
0. \\
00 \\
0
\end{tabular} & $\begin{array}{l}0 . \\
42 \\
9\end{array}$ & $\begin{array}{l}0 . \\
14 \\
3\end{array}$ & $\begin{array}{l}0 . \\
42 \\
9\end{array}$ & \begin{tabular}{|l}
0. \\
00 \\
0
\end{tabular} & $\begin{array}{l}0 . \\
85 \\
7\end{array}$ & $\begin{array}{l}0 . \\
12 \\
5\end{array}$ & \begin{tabular}{|l}
0. \\
00 \\
0
\end{tabular} & $\begin{array}{l}0 . \\
79 \\
9\end{array}$ & $\begin{array}{l}0 . \\
10 \\
5\end{array}$ & c & , & . & & $\begin{array}{l}0 . \\
10 \\
0\end{array}$ & \begin{tabular}{|l}
0. \\
00 \\
0
\end{tabular} & $\begin{array}{l}0 . \\
90 \\
0\end{array}$ & $\begin{array}{l}0 . \\
10 \\
0\end{array}$ & $\begin{array}{l}00 \\
0\end{array}$ & $\begin{array}{l}0 . \\
90 \\
0\end{array}$ & $\begin{array}{l}0 . \\
10 \\
0\end{array}$ & $\begin{array}{l}0 \\
0\end{array}$ & ( & & & & \\
\hline 8 & $\begin{array}{l}0.44 \\
4\end{array}$ & $\begin{array}{l}0 . \\
08 \\
4\end{array}$ & $\begin{array}{l}0 . \\
47 \\
2\end{array}$ & $\begin{array}{l}0 . \\
00 \\
3\end{array}$ & $\begin{array}{l}0 . \\
68 \\
7\end{array}$ & $\begin{array}{l}0 . \\
06 \\
9\end{array}$ & $\begin{array}{l}0 . \\
24 \\
4\end{array}$ & $\begin{array}{l}0 . \\
10 \\
7\end{array}$ & $\begin{array}{l}0 . \\
88 \\
9\end{array}$ & $\begin{array}{l}0 . \\
11 \\
1\end{array}$ & $\begin{array}{l}0 . \\
00 \\
0\end{array}$ & $\begin{array}{l}0 . \\
70 \\
7\end{array}$ & $\begin{array}{l}0 . \\
22 \\
3\end{array}$ & c & . & . & & $\begin{array}{l}0 . \\
11 \\
1\end{array}$ & $\begin{array}{l}0 . \\
00 \\
0\end{array}$ & $\begin{array}{l}0 . \\
87 \\
5\end{array}$ & $\begin{array}{l}0 . \\
12 \\
5\end{array}$ & $\begin{array}{l}00 \\
0\end{array}$ & $\begin{array}{l}0 . \\
88 \\
9\end{array}$ & $\begin{array}{l}0 . \\
11 \\
1\end{array}$ & 0 & 2 & & & & \\
\hline 9 & $\begin{array}{l}0.33 \\
3\end{array}$ & $\begin{array}{l}0 . \\
33 \\
3\end{array}$ & $\begin{array}{l}0 . \\
33 \\
3\end{array}$ & \begin{tabular}{|l}
0. \\
00 \\
0
\end{tabular} & $\begin{array}{l}0 . \\
67 \\
2\end{array}$ & $\begin{array}{l}0 . \\
06 \\
3\end{array}$ & $\begin{array}{l}0 . \\
26 \\
5\end{array}$ & \begin{tabular}{|l}
0. \\
02 \\
5
\end{tabular} & $\begin{array}{l}0 . \\
87 \\
5\end{array}$ & $\begin{array}{l}0 . \\
12 \\
5\end{array}$ & \begin{tabular}{|l}
0. \\
00 \\
0
\end{tabular} & $\begin{array}{l}0 . \\
78 \\
5\end{array}$ & $\begin{array}{l}0 . \\
14 \\
9\end{array}$ & c & 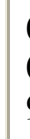 & 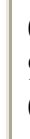 & & $\begin{array}{l}0 . \\
10 \\
0\end{array}$ & \begin{tabular}{|l}
0. \\
00 \\
0
\end{tabular} & $\begin{array}{l}0 . \\
90 \\
0\end{array}$ & $\begin{array}{l}0 . \\
10 \\
0\end{array}$ & $\begin{array}{l}00 \\
0\end{array}$ & $\begin{array}{l}0 . \\
90 \\
0\end{array}$ & $\begin{array}{l}0 . \\
10 \\
0\end{array}$ & 0 & 3 & & & & \\
\hline 10 & $\begin{array}{l}0.33 \\
3\end{array}$ & $\begin{array}{l}0 . \\
33 \\
3\end{array}$ & $\begin{array}{l}0 . \\
33 \\
3\end{array}$ & \begin{tabular}{|l}
0. \\
00 \\
0
\end{tabular} & $\begin{array}{l}0 . \\
66 \\
7\end{array}$ & $\begin{array}{l}0 . \\
16 \\
7\end{array}$ & $\begin{array}{l}0 . \\
16 \\
7\end{array}$ & \begin{tabular}{|l}
0. \\
00 \\
0
\end{tabular} & $\begin{array}{l}0 . \\
85 \\
7\end{array}$ & $\begin{array}{l}0 . \\
14 \\
3\end{array}$ & \begin{tabular}{|l}
0. \\
00 \\
0
\end{tabular} & $\begin{array}{l}0 . \\
76 \\
1\end{array}$ & $\begin{array}{l}0 . \\
1 \epsilon \\
6\end{array}$ & 3 & 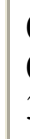 & 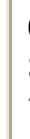 & & $\begin{array}{l}0 . \\
14 \\
3\end{array}$ & \begin{tabular}{|l}
0. \\
00 \\
0
\end{tabular} & \begin{tabular}{|l}
0. \\
85 \\
7
\end{tabular} & $\begin{array}{l}0 . \\
14 \\
3\end{array}$ & $\begin{array}{l}00 \\
0\end{array}$ & \begin{tabular}{|l}
0. \\
88 \\
9
\end{tabular} & $\begin{array}{l}0 . \\
11 \\
1\end{array}$ & 0 & S & & & & \\
\hline 11 & $\begin{array}{l}0.52 \\
8\end{array}$ & $\begin{array}{l}0 . \\
09 \\
1\end{array}$ & $\begin{array}{l}0 . \\
38 \\
1\end{array}$ & \begin{tabular}{|l}
0. \\
09 \\
3
\end{tabular} & $\begin{array}{l}0 . \\
76 \\
1\end{array}$ & $\begin{array}{l}0 . \\
16 \\
6\end{array}$ & $\begin{array}{l}0 . \\
07 \\
3\end{array}$ & $\begin{array}{l}0 . \\
06 \\
3\end{array}$ & $\begin{array}{l}0 . \\
83 \\
3\end{array}$ & $\begin{array}{l}0 . \\
16 \\
7\end{array}$ & \begin{tabular}{|l}
0. \\
00 \\
0
\end{tabular} & $\begin{array}{l}0 . \\
77 \\
0\end{array}$ & $\begin{array}{l}0 . \\
1 \epsilon \\
2\end{array}$ & 8 & ( & 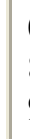 & & $\begin{array}{l}0 . \\
11 \\
1\end{array}$ & \begin{tabular}{|l}
0. \\
00 \\
0
\end{tabular} & $\begin{array}{l}0 . \\
90 \\
0\end{array}$ & $\begin{array}{l}0 . \\
10 \\
0\end{array}$ & $\begin{array}{l}00 \\
0\end{array}$ & \begin{tabular}{|l}
0. \\
88 \\
9
\end{tabular} & $\begin{array}{l}0 . \\
11 \\
1\end{array}$ & 0 & s & & & & \\
\hline $\begin{array}{l}\mathrm{M} \\
\mathrm{ea} \\
\mathrm{n}\end{array}$ & $\begin{array}{l}0.46 \\
1\end{array}$ & $\begin{array}{l}0 . \\
20 \\
3\end{array}$ & $\begin{array}{l}0 . \\
26 \\
1\end{array}$ & - & $\begin{array}{l}0 . \\
51 \\
7\end{array}$ & $\begin{array}{l}0 . \\
14 \\
7\end{array}$ & $\begin{array}{l}0 . \\
25 \\
1\end{array}$ & - & $\begin{array}{l}0 . \\
85 \\
6\end{array}$ & $\begin{array}{l}0 . \\
13 \\
9\end{array}$ & - & $\begin{array}{l}0 . \\
76 \\
2\end{array}$ & $\begin{array}{l}0 . \\
16 \\
0\end{array}$ & 0 & & . & & $\begin{array}{l}0 . \\
10 \\
7\end{array}$ & - & $\begin{array}{l}0 . \\
89 \\
3\end{array}$ & $\begin{array}{l}0 . \\
10 \\
6\end{array}$ & - & $\begin{array}{l}0 . \\
89 \\
5\end{array}$ & $\begin{array}{l}0 . \\
10 \\
5\end{array}$ & - & - & & & & \\
\hline A & 0.48 & 0. & 0. & 0. & 0. & 0. & 0. & 0. & 0. & 0. & 0. & 0. & 0. & 0 & & . & & 0. & 0. & 0. & 0 . & 0 . & 0. & 0. & 0 & ( & & & & \\
\hline
\end{tabular}




\begin{tabular}{|c|c|c|c|c|c|c|c|c|c|c|c|c|c|c|c|c|c|c|c|c|c|c|c|c|c|c|c|c|c|}
\hline \multirow{2}{*}{$\begin{array}{l}\text { Pa } \\
\text { rt. } \\
\text { no } \\
\cdot\end{array}$} & \multicolumn{4}{|c|}{ Level 2.1} & \multicolumn{4}{|c|}{ Level 2.2} & \multicolumn{7}{|c|}{ Level 3.1} & \multicolumn{4}{|c|}{ Level 3.2} & \multicolumn{3}{|c|}{ Level 3.3} & \multicolumn{3}{|c|}{ Level 3.4} & \multicolumn{3}{|c|}{ Level 3.5} & \multirow{2}{*}{$\begin{array}{l}\text { A } \\
\text { vg } \\
\text { C } \\
\text { R }\end{array}$} \\
\hline & $\begin{array}{l}\text { Tec } \\
\text { h/E } \\
\text { q. }\end{array}$ & $\begin{array}{l}\text { In } \\
\text { fr } \\
\text { a. }\end{array}$ & $\begin{array}{l}\text { Pr } \\
\text { oc } \\
\text {. }\end{array}$ & $\begin{array}{l}\mathbf{C} \\
\mathbf{R}\end{array}$ & $\begin{array}{l}\mathbf{E} \\
\mathbf{q} .\end{array}$ & $\begin{array}{l}\text { Tr } \\
\text { an } \\
\text {. }\end{array}$ & $\begin{array}{l}\text { C } \\
\text { on } \\
\text { t. }\end{array}$ & $\begin{array}{l}\mathbf{C} \\
\mathbf{R}\end{array}$ & $\begin{array}{l}\mathbf{M} \\
\mathbf{C} \\
\mathbf{I}\end{array}$ & $\begin{array}{l}\mathbf{S} \\
\mathbf{C} \\
\mathbf{I}\end{array}$ & $\begin{array}{l}\text { S } \\
\boldsymbol{\&} \\
\mathbf{D}\end{array}$ & $\begin{array}{l}\mathbf{W} \\
\mathbf{S}\end{array}$ & $\begin{array}{l}\text { D } \\
\text { S }\end{array}$ & $\mathbf{E}$ & $\begin{array}{l}\mathbf{C} \\
\mathbf{R}\end{array}$ & $\begin{array}{l}\mathbf{E} \\
\mathbf{W} \\
\mathbf{V}\end{array}$ & $\begin{array}{l}\mathbf{G} \\
\mathbf{V}\end{array}$ & $\begin{array}{l}\text { I } \\
\text { V }\end{array}$ & $\begin{array}{l}\mathbf{C} \\
\mathbf{R}\end{array}$ & $\begin{array}{l}\mathbf{E} \\
\mathbf{C}\end{array}$ & $\begin{array}{l}\mathbf{n} \\
\mathbf{E} \\
\mathbf{C}\end{array}$ & $\begin{array}{l}\mathbf{C} \\
\mathbf{R}\end{array}$ & $\begin{array}{l}\mathbf{E} \\
\mathbf{S}\end{array}$ & $\begin{array}{l}\mathbf{n} \\
\mathbf{E} \\
\mathbf{S}\end{array}$ & $\begin{array}{l}\mathbf{C} \\
\mathbf{R}\end{array}$ & $\begin{array}{l}\mathbf{D} \\
\mathbf{P}\end{array}$ & $\begin{array}{l}\mathbf{n} \\
\mathbf{P}\end{array}$ & $\begin{array}{l}\mathbf{C} \\
\mathbf{R}\end{array}$ & \\
\hline $\begin{array}{l}\mathrm{vg} \\
.\end{array}$ & 7 & $\begin{array}{l}22 \\
8\end{array}$ & $\begin{array}{l}28 \\
5\end{array}$ & $\begin{array}{l}01 \\
6\end{array}$ & $\begin{array}{l}55 \\
2\end{array}$ & $\begin{array}{l}15 \\
8\end{array}$ & $\begin{array}{l}29 \\
0\end{array}$ & $\begin{array}{l}02 \\
2\end{array}$ & $\begin{array}{l}85 \\
6\end{array}$ & $\begin{array}{l}14 \\
1\end{array}$ & $\begin{array}{l}00 \\
0\end{array}$ & $\begin{array}{l}76 \\
2\end{array}$ & $\begin{array}{l}16 \\
4\end{array}$ & $\begin{array}{l}07 \\
3\end{array}$ & $\begin{array}{l}04 \\
5\end{array}$ & $\begin{array}{l}89 \\
2\end{array}$ & $\begin{array}{l}10 \\
8\end{array}$ & $\begin{array}{l}00 \\
0\end{array}$ & $\begin{array}{l}89 \\
3\end{array}$ & $\begin{array}{l}10 \\
7\end{array}$ & $\begin{array}{l}00 \\
0\end{array}$ & $\begin{array}{l}89 \\
5\end{array}$ & $\begin{array}{l}10 \\
5\end{array}$ & $\begin{array}{l}00 \\
0\end{array}$ & $\begin{array}{l}01 \\
2\end{array}$ & & & & \\
\hline $\begin{array}{l}\text { St } \\
\text { d. } \\
\text { de } \\
\text { v. }\end{array}$ & $\begin{array}{l}0.16 \\
7\end{array}$ & $\begin{array}{l}0 . \\
10 \\
6\end{array}$ & $\begin{array}{l}0 . \\
11 \\
3\end{array}$ & $\begin{array}{l}0 . \\
03 \\
5\end{array}$ & $\begin{array}{l}0 . \\
18 \\
2\end{array}$ & $\begin{array}{l}0 . \\
05 \\
9\end{array}$ & $\begin{array}{l}0 . \\
15 \\
0\end{array}$ & $\begin{array}{l}0 . \\
03 \\
6\end{array}$ & $\begin{array}{l}0 . \\
02 \\
1\end{array}$ & $\begin{array}{l}0 . \\
02 \\
2\end{array}$ & $\begin{array}{l}0 . \\
00 \\
0\end{array}$ & $\begin{array}{l}0 . \\
02 \\
7\end{array}$ & $\begin{array}{l}0 . \\
03 \\
6\end{array}$ & $\begin{array}{l}0 . \\
01 \\
2\end{array}$ & $\begin{array}{l}0 . \\
02 \\
7\end{array}$ & $\begin{array}{l}0 . \\
01 \\
4\end{array}$ & $\begin{array}{l}0 . \\
01 \\
4\end{array}$ & $\begin{array}{l}0 . \\
00 \\
0\end{array}$ & $\begin{array}{l}0 . \\
01 \\
4\end{array}$ & $\begin{array}{l}0 . \\
01 \\
4\end{array}$ & $\begin{array}{l}0 . \\
00 \\
0\end{array}$ & $\begin{array}{l}0 . \\
00 \\
8\end{array}$ & $\begin{array}{l}0 . \\
00 \\
8\end{array}$ & $\begin{array}{l}0 . \\
00 \\
0\end{array}$ & $\begin{array}{l}0 . \\
01 \\
1\end{array}$ & & & & \\
\hline
\end{tabular}

\section{Table 9.}

AHP results for the waste disposal life cycle phase (central)

\begin{tabular}{|c|c|c|c|c|c|c|c|c|c|c|c|c|c|c|c|c|c|c|c|c|c|c|}
\hline \multirow{2}{*}{$\begin{array}{l}\text { Par } \\
\text { t. } \\
\text { no. }\end{array}$} & \multicolumn{3}{|c|}{ Level 2.1} & \multicolumn{4}{|c|}{ Level 2.2} & \multicolumn{4}{|c|}{ Level 3.1} & \multicolumn{4}{|c|}{ Level 3.2} & \multicolumn{3}{|c|}{ Level 3.3} & \multicolumn{3}{|c|}{ Level 3.4} & \multirow{2}{*}{$\begin{array}{l}\text { Avg } \\
\dot{C R}\end{array}$} \\
\hline & $\begin{array}{l}\text { Tech./ } \\
\text { Eq. }\end{array}$ & $\begin{array}{l}\text { Pro } \\
\text { C. }\end{array}$ & CR & Eq. & $\begin{array}{l}\text { Tra } \\
\text { n. }\end{array}$ & $\begin{array}{l}\text { Con } \\
\text { t. }\end{array}$ & CR & OD & CD & LF & $\mathbf{C R}$ & $\begin{array}{l}\text { EW } \\
\mathbf{V}\end{array}$ & $\mathbf{G V}$ & IV & CR & EC & $\begin{array}{l}\mathbf{n E} \\
\mathrm{C}\end{array}$ & CR & DP & $\mathbf{n P}$ & CR & \\
\hline \multicolumn{23}{|c|}{ South African workshop } \\
\hline 1 & 0.889 & $\begin{array}{l}0.1 \\
11\end{array}$ & $\begin{array}{l}0.0 \\
00\end{array}$ & $\begin{array}{l}0.7 \\
72\end{array}$ & $\begin{array}{l}0.05 \\
3\end{array}$ & $\begin{array}{l}0.17 \\
5\end{array}$ & $\begin{array}{l}0.3 \\
17\end{array}$ & $\begin{array}{l}0.0 \\
52\end{array}$ & $\begin{array}{l}0.1 \\
65\end{array}$ & $\begin{array}{l}0.7 \\
84\end{array}$ & $\begin{array}{l}0.3 \\
61\end{array}$ & $\begin{array}{l}0.81 \\
4\end{array}$ & $\begin{array}{l}0.1 \\
14\end{array}$ & $\begin{array}{l}0.0 \\
72\end{array}$ & $\begin{array}{l}0.0 \\
46\end{array}$ & $\begin{array}{l}0.9 \\
00\end{array}$ & $\begin{array}{l}0.1 \\
00\end{array}$ & $\begin{array}{l}0.0 \\
00\end{array}$ & $\begin{array}{l}0.8 \\
89\end{array}$ & $\begin{array}{l}0.1 \\
11\end{array}$ & $\begin{array}{l}0.0 \\
00\end{array}$ & $\begin{array}{l}0.1 \\
21\end{array}$ \\
\hline 2 & 0.500 & $\begin{array}{l}0.5 \\
00\end{array}$ & $\begin{array}{l}0.0 \\
00\end{array}$ & $\begin{array}{l}0.5 \\
28\end{array}$ & $\begin{array}{l}0.22 \\
7\end{array}$ & $\begin{array}{l}0.24 \\
5\end{array}$ & $\begin{array}{l}0.5 \\
33\end{array}$ & $\begin{array}{l}0.0 \\
50\end{array}$ & $\begin{array}{l}0.1 \\
70\end{array}$ & $\begin{array}{l}0.7 \\
80\end{array}$ & $\begin{array}{l}0.2 \\
75\end{array}$ & $\begin{array}{l}0.77 \\
0\end{array}$ & $\begin{array}{l}0.1 \\
85\end{array}$ & $\begin{array}{l}0.0 \\
45\end{array}$ & $\begin{array}{l}0.3 \\
82\end{array}$ & $\begin{array}{l}0.9 \\
00\end{array}$ & $\begin{array}{l}0.1 \\
00\end{array}$ & $\begin{array}{l}0.0 \\
00\end{array}$ & $\begin{array}{l}0.9 \\
00\end{array}$ & $\begin{array}{l}0.1 \\
00\end{array}$ & $\begin{array}{l}0.0 \\
00\end{array}$ & $\begin{array}{l}0.1 \\
98\end{array}$ \\
\hline
\end{tabular}




\begin{tabular}{|c|c|c|c|c|c|c|c|c|c|c|c|c|c|c|c|c|c|c|c|c|c|c|}
\hline \multirow{2}{*}{$\begin{array}{l}\text { Par } \\
\text { t. } \\
\text { no. }\end{array}$} & \multicolumn{3}{|c|}{ Level 2.1} & \multicolumn{4}{|c|}{ Level 2.2} & \multicolumn{4}{|c|}{ Level 3.1} & \multicolumn{4}{|c|}{ Level 3.2} & \multicolumn{3}{|c|}{ Level 3.3} & \multicolumn{3}{|c|}{ Level 3.4} & \multirow{2}{*}{$\begin{array}{l}\text { Avg } \\
\text { CR }\end{array}$} \\
\hline & $\begin{array}{l}\text { Tech./ } \\
\text { Eq. }\end{array}$ & $\begin{array}{l}\text { Pro } \\
\text { c. }\end{array}$ & CR & Eq. & $\begin{array}{l}\text { Tra } \\
\text { n. }\end{array}$ & $\begin{array}{l}\text { Con } \\
\text { t. }\end{array}$ & CR & OD & CD & $\mathbf{L F}$ & CR & $\begin{array}{l}\text { EW } \\
\mathbf{V}\end{array}$ & GV & IV & CR & EC & $\begin{array}{l}\mathbf{n E} \\
\mathrm{C}\end{array}$ & CR & DP & $\mathbf{n P}$ & CR & \\
\hline 3 & 0.500 & $\begin{array}{l}0.5 \\
00\end{array}$ & $\begin{array}{l}0.0 \\
00\end{array}$ & $\begin{array}{l}0.5 \\
71\end{array}$ & $\begin{array}{l}0.14 \\
3\end{array}$ & $\begin{array}{l}0.28 \\
6\end{array}$ & $\begin{array}{l}0.4 \\
31\end{array}$ & $\begin{array}{l}0.0 \\
48\end{array}$ & $\begin{array}{l}0.1 \\
60\end{array}$ & $\begin{array}{l}0.7 \\
92\end{array}$ & $\begin{array}{l}0.3 \\
17\end{array}$ & $\begin{array}{l}0.79 \\
7\end{array}$ & $\begin{array}{l}0.1 \\
51\end{array}$ & $\begin{array}{l}0.0 \\
52\end{array}$ & $\begin{array}{l}0.2 \\
54\end{array}$ & $\begin{array}{l}0.9 \\
00\end{array}$ & $\begin{array}{l}0.1 \\
00\end{array}$ & $\begin{array}{l}0.0 \\
00\end{array}$ & $\begin{array}{l}0.8 \\
57\end{array}$ & $\begin{array}{l}0.1 \\
43\end{array}$ & $\begin{array}{l}0.0 \\
00\end{array}$ & $\begin{array}{l}0.1 \\
67\end{array}$ \\
\hline 4 & 0.667 & $\begin{array}{l}0.3 \\
33\end{array}$ & $\begin{array}{l}0.0 \\
00\end{array}$ & $\begin{array}{l}0.7 \\
07\end{array}$ & $\begin{array}{l}0.07 \\
0\end{array}$ & $\begin{array}{l}0.22 \\
3\end{array}$ & $\begin{array}{l}0.7 \\
90\end{array}$ & $\begin{array}{l}0.0 \\
54\end{array}$ & $\begin{array}{l}0.2 \\
33\end{array}$ & $\begin{array}{l}0.7 \\
12\end{array}$ & $\begin{array}{l}0.2 \\
13\end{array}$ & $\begin{array}{l}0.75 \\
6\end{array}$ & $\begin{array}{l}0.1 \\
88\end{array}$ & $\begin{array}{l}0.0 \\
56\end{array}$ & $\begin{array}{l}0.1 \\
41\end{array}$ & $\begin{array}{l}0.8 \\
89\end{array}$ & $\begin{array}{l}0.1 \\
11\end{array}$ & $\begin{array}{l}0.0 \\
00\end{array}$ & $\begin{array}{l}0.9 \\
00\end{array}$ & $\begin{array}{l}0.1 \\
00\end{array}$ & $\begin{array}{l}0.0 \\
00\end{array}$ & $\begin{array}{l}0.1 \\
91\end{array}$ \\
\hline 5 & 0.500 & $\begin{array}{l}0.5 \\
00\end{array}$ & $\begin{array}{l}0.0 \\
00\end{array}$ & $\begin{array}{l}0.7 \\
14\end{array}$ & $\begin{array}{l}0.14 \\
3\end{array}$ & $\begin{array}{l}0.14 \\
3\end{array}$ & $\begin{array}{l}0.0 \\
00\end{array}$ & $\begin{array}{l}0.1 \\
77\end{array}$ & $\begin{array}{l}0.5 \\
19\end{array}$ & $\begin{array}{l}0.3 \\
04\end{array}$ & $\begin{array}{l}0.2 \\
54\end{array}$ & $\begin{array}{l}0.76 \\
3\end{array}$ & $\begin{array}{l}0.1 \\
78\end{array}$ & $\begin{array}{l}0.0 \\
58\end{array}$ & $\begin{array}{l}0.2 \\
13\end{array}$ & $\begin{array}{l}0.9 \\
00\end{array}$ & $\begin{array}{l}0.1 \\
00\end{array}$ & $\begin{array}{l}0.0 \\
00\end{array}$ & $\begin{array}{l}0.9 \\
00\end{array}$ & $\begin{array}{l}0.1 \\
00\end{array}$ & $\begin{array}{l}0.0 \\
00\end{array}$ & $\begin{array}{l}0.0 \\
78\end{array}$ \\
\hline 6 & 0.900 & $\begin{array}{l}0.1 \\
00\end{array}$ & $\begin{array}{l}0.0 \\
00\end{array}$ & $\begin{array}{l}0.3 \\
33\end{array}$ & $\begin{array}{l}0.33 \\
3\end{array}$ & $\begin{array}{l}0.33 \\
3\end{array}$ & $\begin{array}{l}0.0 \\
00\end{array}$ & $\begin{array}{l}0.0 \\
65\end{array}$ & $\begin{array}{l}0.2 \\
04\end{array}$ & $\begin{array}{l}0.7 \\
30\end{array}$ & $\begin{array}{l}0.5 \\
90\end{array}$ & $\begin{array}{l}0.79 \\
7\end{array}$ & $\begin{array}{l}0.1 \\
51\end{array}$ & $\begin{array}{l}0.0 \\
52\end{array}$ & $\begin{array}{l}0.2 \\
54\end{array}$ & $\begin{array}{l}0.8 \\
89\end{array}$ & $\begin{array}{l}0.1 \\
11\end{array}$ & $\begin{array}{l}0.0 \\
00\end{array}$ & $\begin{array}{l}0.9 \\
00\end{array}$ & $\begin{array}{l}0.1 \\
00\end{array}$ & $\begin{array}{l}0.0 \\
00\end{array}$ & $\begin{array}{l}0.1 \\
41\end{array}$ \\
\hline 7 & 0.750 & $\begin{array}{l}0.2 \\
50\end{array}$ & $\begin{array}{l}0.0 \\
00\end{array}$ & $\begin{array}{l}0.3 \\
33\end{array}$ & $\begin{array}{l}0.33 \\
3\end{array}$ & $\begin{array}{l}0.33 \\
3\end{array}$ & $\begin{array}{l}0.0 \\
00\end{array}$ & $\begin{array}{l}0.0 \\
47\end{array}$ & $\begin{array}{l}0.1 \\
78\end{array}$ & $\begin{array}{l}0.7 \\
75\end{array}$ & $\begin{array}{l}0.3 \\
30\end{array}$ & $\begin{array}{l}0.78 \\
7\end{array}$ & $\begin{array}{l}0.1 \\
67\end{array}$ & $\begin{array}{l}0.0 \\
46\end{array}$ & $\begin{array}{l}0.3 \\
76\end{array}$ & $\begin{array}{l}0.9 \\
00\end{array}$ & $\begin{array}{l}0.1 \\
00\end{array}$ & $\begin{array}{l}0.0 \\
00\end{array}$ & $\begin{array}{l}0.9 \\
00\end{array}$ & $\begin{array}{l}0.1 \\
00\end{array}$ & $\begin{array}{l}0.0 \\
00\end{array}$ & $\begin{array}{l}0.1 \\
18\end{array}$ \\
\hline 8 & 0.500 & $\begin{array}{l}0.5 \\
00\end{array}$ & $\begin{array}{l}0.0 \\
00\end{array}$ & $\begin{array}{l}0.3 \\
33\end{array}$ & $\begin{array}{l}0.33 \\
3\end{array}$ & $\begin{array}{l}0.33 \\
3\end{array}$ & $\begin{array}{l}0.0 \\
00\end{array}$ & $\begin{array}{l}0.0 \\
75\end{array}$ & $\begin{array}{l}0.3 \\
57\end{array}$ & $\begin{array}{l}0.5 \\
67\end{array}$ & $\begin{array}{l}0.0 \\
46\end{array}$ & $\begin{array}{l}0.76 \\
1\end{array}$ & $\begin{array}{l}0.1 \\
66\end{array}$ & $\begin{array}{l}0.0 \\
73\end{array}$ & $\begin{array}{l}0.0 \\
63\end{array}$ & $\begin{array}{l}0.9 \\
00\end{array}$ & $\begin{array}{l}0.1 \\
00\end{array}$ & $\begin{array}{l}0.0 \\
00\end{array}$ & $\begin{array}{l}0.9 \\
00\end{array}$ & $\begin{array}{l}0.1 \\
00\end{array}$ & $\begin{array}{l}0.0 \\
00\end{array}$ & $\begin{array}{l}0.0 \\
18\end{array}$ \\
\hline 9 & 0.800 & $\begin{array}{l}0.2 \\
00\end{array}$ & $\begin{array}{l}0.0 \\
00\end{array}$ & $\begin{array}{l}0.7 \\
15\end{array}$ & $\begin{array}{l}0.09 \\
8\end{array}$ & $\begin{array}{l}0.18 \\
7\end{array}$ & $\begin{array}{l}0.0 \\
02\end{array}$ & $\begin{array}{l}0.1 \\
02\end{array}$ & $\begin{array}{l}0.1 \\
72\end{array}$ & $\begin{array}{l}0.7 \\
26\end{array}$ & $\begin{array}{l}0.0 \\
25\end{array}$ & $\begin{array}{l}0.70 \\
9\end{array}$ & $\begin{array}{l}0.2 \\
12\end{array}$ & $\begin{array}{l}0.0 \\
79\end{array}$ & $\begin{array}{l}0.1 \\
41\end{array}$ & $\begin{array}{l}0.8 \\
00\end{array}$ & $\begin{array}{l}0.2 \\
00\end{array}$ & $\begin{array}{l}0.0 \\
00\end{array}$ & $\begin{array}{l}0.8 \\
00\end{array}$ & $\begin{array}{l}0.2 \\
00\end{array}$ & $\begin{array}{l}0.0 \\
00\end{array}$ & $\begin{array}{l}0.0 \\
28\end{array}$ \\
\hline 10 & 0.167 & $\begin{array}{l}0.8 \\
33\end{array}$ & $\begin{array}{l}0.0 \\
00\end{array}$ & $\begin{array}{l}0.4 \\
55\end{array}$ & $\begin{array}{l}0.09 \\
1\end{array}$ & $\begin{array}{l}0.45 \\
5\end{array}$ & $\begin{array}{l}0.0 \\
00\end{array}$ & $\begin{array}{l}0.0 \\
63\end{array}$ & $\begin{array}{l}0.2 \\
65\end{array}$ & $\begin{array}{l}0.6 \\
72\end{array}$ & $\begin{array}{l}0.0 \\
25\end{array}$ & $\begin{array}{l}0.73 \\
1\end{array}$ & $\begin{array}{l}0.1 \\
88\end{array}$ & $\begin{array}{l}0.0 \\
81\end{array}$ & $\begin{array}{l}0.0 \\
56\end{array}$ & $\begin{array}{l}0.8 \\
75\end{array}$ & $\begin{array}{l}0.1 \\
25\end{array}$ & $\begin{array}{l}0.0 \\
00\end{array}$ & $\begin{array}{l}0.8 \\
75\end{array}$ & $\begin{array}{l}0.1 \\
25\end{array}$ & $\begin{array}{l}0.0 \\
00\end{array}$ & $\begin{array}{l}0.0 \\
13\end{array}$ \\
\hline 11 & 0.500 & $\begin{array}{l}0.5 \\
00\end{array}$ & $\begin{array}{l}0.0 \\
00\end{array}$ & $\begin{array}{l}0.7 \\
47\end{array}$ & $\begin{array}{l}0.04 \\
9\end{array}$ & $\begin{array}{l}0.20 \\
4\end{array}$ & $\begin{array}{l}0.3 \\
76\end{array}$ & $\begin{array}{l}0.0 \\
48\end{array}$ & $\begin{array}{l}0.2 \\
71\end{array}$ & $\begin{array}{l}0.6 \\
82\end{array}$ & $\begin{array}{l}0.1 \\
87\end{array}$ & $\begin{array}{l}0.75 \\
1\end{array}$ & $\begin{array}{l}0.2 \\
05\end{array}$ & $\begin{array}{l}0.0 \\
44\end{array}$ & $\begin{array}{l}0.3 \\
76\end{array}$ & $\begin{array}{l}0.9 \\
00\end{array}$ & $\begin{array}{l}0.1 \\
00\end{array}$ & $\begin{array}{l}0.0 \\
00\end{array}$ & $\begin{array}{l}0.9 \\
00\end{array}$ & $\begin{array}{l}0.1 \\
00\end{array}$ & $\begin{array}{l}0.0 \\
00\end{array}$ & $\begin{array}{l}0.1 \\
56\end{array}$ \\
\hline $\begin{array}{l}\mathrm{Me} \\
\text { an }\end{array}$ & 0.559 & $\begin{array}{l}0.3 \\
29\end{array}$ & - & $\begin{array}{l}0.4 \\
52\end{array}$ & $\begin{array}{l}0.19 \\
0\end{array}$ & $\begin{array}{l}0.27 \\
7\end{array}$ & - & $\begin{array}{l}0.0 \\
78\end{array}$ & $\begin{array}{l}0.2 \\
54\end{array}$ & $\begin{array}{l}0.6 \\
52\end{array}$ & - & $\begin{array}{l}0.76 \\
8\end{array}$ & $\begin{array}{l}0.1 \\
53\end{array}$ & $\begin{array}{l}0.0 \\
75\end{array}$ & - & $\begin{array}{l}0.8 \\
86\end{array}$ & $\begin{array}{l}0.1 \\
11\end{array}$ & - & $\begin{array}{l}0.8 \\
83\end{array}$ & $\begin{array}{l}0.1 \\
13\end{array}$ & - & - \\
\hline Avg & 0.607 & 0.3 & 0.0 & 0.4 & 0.22 & 0.29 & 0.0 & 0.0 & 0.2 & 0.6 & 0.0 & 0.76 & 0.1 & 0.0 & 0.0 & 0.8 & 0.1 & 0.0 & 0.8 & 0.1 & 0.0 & 0.1 \\
\hline
\end{tabular}




\begin{tabular}{|c|c|c|c|c|c|c|c|c|c|c|c|c|c|c|c|c|c|c|c|c|c|c|}
\hline \multirow{2}{*}{$\begin{array}{l}\text { Par } \\
\text { t. } \\
\text { no. }\end{array}$} & \multicolumn{3}{|c|}{ Level 2.1} & \multicolumn{4}{|c|}{ Level 2.2} & \multicolumn{4}{|c|}{ Level 3.1} & \multicolumn{4}{|c|}{ Level 3.2} & \multicolumn{3}{|c|}{ Level 3.3} & \multicolumn{3}{|c|}{ Level 3.4} & \multirow{2}{*}{$\begin{array}{l}\text { Avg } \\
\dot{\text { CR }}\end{array}$} \\
\hline & $\begin{array}{l}\text { Tech./ } \\
\text { Eq. }\end{array}$ & $\begin{array}{l}\text { Pro } \\
\text { c. }\end{array}$ & CR & Eq. & $\begin{array}{l}\text { Tra } \\
\text { n. }\end{array}$ & $\begin{array}{l}\text { Con } \\
\text { t. }\end{array}$ & CR & OD & CD & LF & CR & $\begin{array}{l}\text { EW } \\
\text { V }\end{array}$ & GV & IV & CR & EC & $\begin{array}{l}\mathbf{n E} \\
\mathrm{C}\end{array}$ & CR & DP & $\mathbf{n P}$ & CR & \\
\hline . & & 93 & 00 & 81 & 2 & 7 & 00 & 80 & 65 & 55 & 32 & 9 & 56 & 75 & 55 & 87 & 13 & 00 & 84 & 16 & 00 & 21 \\
\hline $\begin{array}{l}\text { Std. } \\
\text { dev. }\end{array}$ & 0.219 & $\begin{array}{l}0.2 \\
19\end{array}$ & $\begin{array}{l}0.0 \\
00\end{array}$ & $\begin{array}{l}0.1 \\
87\end{array}$ & $\begin{array}{l}0.12 \\
3\end{array}$ & $\begin{array}{l}0.11 \\
4\end{array}$ & $\begin{array}{l}0.0 \\
01\end{array}$ & $\begin{array}{l}0.0 \\
20\end{array}$ & $\begin{array}{l}0.0 \\
93\end{array}$ & $\begin{array}{l}0.0 \\
81\end{array}$ & $\begin{array}{l}0.0 \\
12\end{array}$ & $\begin{array}{l}0.04 \\
2\end{array}$ & $\begin{array}{l}0.0 \\
38\end{array}$ & $\begin{array}{l}0.0 \\
05\end{array}$ & $\begin{array}{l}0.0 \\
09\end{array}$ & $\begin{array}{l}0.0 \\
30\end{array}$ & $\begin{array}{l}0.0 \\
30\end{array}$ & $\begin{array}{l}0.0 \\
00\end{array}$ & $\begin{array}{l}0.0 \\
31\end{array}$ & $\begin{array}{l}0.0 \\
31\end{array}$ & $\begin{array}{l}0.0 \\
00\end{array}$ & $\begin{array}{l}0.0 \\
64\end{array}$ \\
\hline \multicolumn{23}{|c|}{ Lesotho workshop } \\
\hline 1 & 0.500 & $\begin{array}{l}0.5 \\
00\end{array}$ & $\begin{array}{l}0.0 \\
00\end{array}$ & $\begin{array}{l}0.7 \\
61\end{array}$ & $\begin{array}{l}0.07 \\
3\end{array}$ & $\begin{array}{l}0.16 \\
6\end{array}$ & $\begin{array}{l}0.0 \\
63\end{array}$ & $\begin{array}{l}0.0 \\
67\end{array}$ & $\begin{array}{l}0.4 \\
67\end{array}$ & $\begin{array}{l}0.4 \\
67\end{array}$ & $\begin{array}{l}0.0 \\
00\end{array}$ & $\begin{array}{l}0.77 \\
7\end{array}$ & $\begin{array}{l}0.1 \\
53\end{array}$ & $\begin{array}{l}0.0 \\
70\end{array}$ & $\begin{array}{l}0.0 \\
90\end{array}$ & $\begin{array}{l}0.8 \\
89\end{array}$ & $\begin{array}{l}0.1 \\
11\end{array}$ & $\begin{array}{l}0.0 \\
00\end{array}$ & $\begin{array}{l}0.8 \\
75\end{array}$ & $\begin{array}{l}0.1 \\
25\end{array}$ & $\begin{array}{l}0.0 \\
00\end{array}$ & $\begin{array}{l}0.0 \\
26\end{array}$ \\
\hline 2 & 0.500 & $\begin{array}{l}0.5 \\
00\end{array}$ & $\begin{array}{l}0.0 \\
00\end{array}$ & $\begin{array}{l}0.8 \\
00\end{array}$ & $\begin{array}{l}0.10 \\
0\end{array}$ & $\begin{array}{l}0.10 \\
0\end{array}$ & $\begin{array}{l}0.0 \\
00\end{array}$ & $\begin{array}{l}0.0 \\
78\end{array}$ & $\begin{array}{l}0.1 \\
71\end{array}$ & $\begin{array}{l}0.7 \\
50\end{array}$ & $\begin{array}{l}0.0 \\
86\end{array}$ & $\begin{array}{l}0.79 \\
8\end{array}$ & $\begin{array}{l}0.1 \\
38\end{array}$ & $\begin{array}{l}0.0 \\
64\end{array}$ & $\begin{array}{l}0.0 \\
93\end{array}$ & $\begin{array}{l}0.8 \\
89\end{array}$ & $\begin{array}{l}0.1 \\
11\end{array}$ & $\begin{array}{l}0.0 \\
00\end{array}$ & $\begin{array}{l}0.8 \\
75\end{array}$ & $\begin{array}{l}0.1 \\
25\end{array}$ & $\begin{array}{l}0.0 \\
00\end{array}$ & $\begin{array}{l}0.0 \\
30\end{array}$ \\
\hline 3 & 0.500 & $\begin{array}{l}0.5 \\
00\end{array}$ & $\begin{array}{l}0.0 \\
00\end{array}$ & $\begin{array}{l}0.8 \\
09\end{array}$ & $\begin{array}{l}0.09 \\
7\end{array}$ & $\begin{array}{l}0.09 \\
4\end{array}$ & $\begin{array}{l}0.0 \\
01\end{array}$ & $\begin{array}{l}0.0 \\
78\end{array}$ & $\begin{array}{l}0.1 \\
71\end{array}$ & $\begin{array}{l}0.7 \\
50\end{array}$ & $\begin{array}{l}0.0 \\
86\end{array}$ & $\begin{array}{l}0.79 \\
8\end{array}$ & $\begin{array}{l}0.1 \\
38\end{array}$ & $\begin{array}{l}0.0 \\
64\end{array}$ & $\begin{array}{l}0.0 \\
93\end{array}$ & $\begin{array}{l}0.8 \\
89\end{array}$ & $\begin{array}{l}0.1 \\
11\end{array}$ & $\begin{array}{l}0.0 \\
00\end{array}$ & $\begin{array}{l}0.8 \\
89\end{array}$ & $\begin{array}{l}0.1 \\
11\end{array}$ & $\begin{array}{l}0.0 \\
00\end{array}$ & $\begin{array}{l}0.0 \\
30\end{array}$ \\
\hline 4 & 0.500 & $\begin{array}{l}0.5 \\
00\end{array}$ & $\begin{array}{l}0.0 \\
00\end{array}$ & $\begin{array}{l}0.2 \\
50\end{array}$ & $\begin{array}{l}0.25 \\
0\end{array}$ & $\begin{array}{l}0.50 \\
0\end{array}$ & $\begin{array}{l}0.0 \\
00\end{array}$ & $\begin{array}{l}0.0 \\
58\end{array}$ & $\begin{array}{l}0.2 \\
07\end{array}$ & $\begin{array}{l}0.7 \\
35\end{array}$ & $\begin{array}{l}0.1 \\
01\end{array}$ & $\begin{array}{l}0.76 \\
1\end{array}$ & $\begin{array}{l}0.1 \\
58\end{array}$ & $\begin{array}{l}0.0 \\
82\end{array}$ & $\begin{array}{l}0.0 \\
01\end{array}$ & $\begin{array}{l}0.9 \\
00\end{array}$ & $\begin{array}{l}0.1 \\
00\end{array}$ & $\begin{array}{l}0.0 \\
00\end{array}$ & $\begin{array}{l}0.9 \\
00\end{array}$ & $\begin{array}{l}0.1 \\
00\end{array}$ & $\begin{array}{l}0.0 \\
00\end{array}$ & $\begin{array}{l}0.0 \\
17\end{array}$ \\
\hline 5 & 0.500 & $\begin{array}{l}0.5 \\
00\end{array}$ & $\begin{array}{l}0.0 \\
00\end{array}$ & $\begin{array}{l}0.2 \\
50\end{array}$ & $\begin{array}{l}0.25 \\
0\end{array}$ & $\begin{array}{l}0.50 \\
0\end{array}$ & $\begin{array}{l}0.0 \\
00\end{array}$ & $\begin{array}{l}0.0 \\
58\end{array}$ & $\begin{array}{l}0.2 \\
07\end{array}$ & $\begin{array}{l}0.7 \\
35\end{array}$ & $\begin{array}{l}0.1 \\
01\end{array}$ & $\begin{array}{l}0.76 \\
1\end{array}$ & $\begin{array}{l}0.1 \\
58\end{array}$ & $\begin{array}{l}0.0 \\
82\end{array}$ & $\begin{array}{l}0.0 \\
01\end{array}$ & $\begin{array}{l}0.9 \\
00\end{array}$ & $\begin{array}{l}0.1 \\
00\end{array}$ & $\begin{array}{l}0.0 \\
00\end{array}$ & $\begin{array}{l}0.9 \\
00\end{array}$ & $\begin{array}{l}0.1 \\
00\end{array}$ & $\begin{array}{l}0.0 \\
00\end{array}$ & $\begin{array}{l}0.0 \\
17\end{array}$ \\
\hline 6 & 0.500 & $\begin{array}{l}0.5 \\
00\end{array}$ & $\begin{array}{l}0.0 \\
00\end{array}$ & $\begin{array}{l}0.4 \\
58\end{array}$ & $\begin{array}{l}0.06 \\
3\end{array}$ & $\begin{array}{l}0.47 \\
9\end{array}$ & $\begin{array}{l}0.0 \\
02\end{array}$ & $\begin{array}{l}0.0 \\
74\end{array}$ & $\begin{array}{l}0.1 \\
22\end{array}$ & $\begin{array}{l}0.8 \\
04\end{array}$ & $\begin{array}{l}0.0 \\
32\end{array}$ & $\begin{array}{l}0.81 \\
8\end{array}$ & $\begin{array}{l}0.0 \\
91\end{array}$ & $\begin{array}{l}0.0 \\
91\end{array}$ & $\begin{array}{l}0.0 \\
00\end{array}$ & $\begin{array}{l}0.9 \\
00\end{array}$ & $\begin{array}{l}0.1 \\
00\end{array}$ & $\begin{array}{l}0.0 \\
00\end{array}$ & $\begin{array}{l}0.9 \\
00\end{array}$ & $\begin{array}{l}0.1 \\
00\end{array}$ & $\begin{array}{l}0.0 \\
00\end{array}$ & $\begin{array}{l}0.0 \\
06\end{array}$ \\
\hline 7 & 0.500 & $\begin{array}{l}0.5 \\
00\end{array}$ & $\begin{array}{l}0.0 \\
00\end{array}$ & $\begin{array}{l}0.4 \\
29\end{array}$ & $\begin{array}{l}0.14 \\
3\end{array}$ & $\begin{array}{l}0.42 \\
9\end{array}$ & $\begin{array}{l}0.0 \\
00\end{array}$ & $\begin{array}{l}0.0 \\
75\end{array}$ & $\begin{array}{l}0.3 \\
33\end{array}$ & $\begin{array}{l}0.5 \\
92\end{array}$ & $\begin{array}{l}0.0 \\
12\end{array}$ & $\begin{array}{l}0.77 \\
7\end{array}$ & $\begin{array}{l}0.1 \\
53\end{array}$ & $\begin{array}{l}0.0 \\
70\end{array}$ & $\begin{array}{l}0.0 \\
90\end{array}$ & $\begin{array}{l}0.8 \\
89\end{array}$ & $\begin{array}{l}0.1 \\
11\end{array}$ & $\begin{array}{l}0.0 \\
00\end{array}$ & $\begin{array}{l}0.8 \\
89\end{array}$ & $\begin{array}{l}0.1 \\
11\end{array}$ & $\begin{array}{l}0.0 \\
00\end{array}$ & $\begin{array}{l}0.0 \\
17\end{array}$ \\
\hline 8 & 0.500 & $\begin{array}{l}0.5 \\
00\end{array}$ & $\begin{array}{l}0.0 \\
00\end{array}$ & $\begin{array}{l}0.3 \\
33\end{array}$ & $\begin{array}{l}0.33 \\
3\end{array}$ & $\begin{array}{l}0.33 \\
3\end{array}$ & $\begin{array}{l}0.0 \\
00\end{array}$ & $\begin{array}{l}0.0 \\
51\end{array}$ & $\begin{array}{l}0.2 \\
22\end{array}$ & $\begin{array}{l}0.7 \\
22\end{array}$ & $\begin{array}{l}\mathbf{0 . 1} \\
80\end{array}$ & $\begin{array}{l}0.79 \\
8\end{array}$ & $\begin{array}{l}0.1 \\
38\end{array}$ & $\begin{array}{l}0.0 \\
64\end{array}$ & $\begin{array}{l}0.0 \\
93\end{array}$ & $\begin{array}{l}0.8 \\
89\end{array}$ & $\begin{array}{l}0.1 \\
11\end{array}$ & $\begin{array}{l}0.0 \\
00\end{array}$ & $\begin{array}{l}0.8 \\
89\end{array}$ & $\begin{array}{l}0.1 \\
11\end{array}$ & $\begin{array}{l}0.0 \\
00\end{array}$ & $\begin{array}{l}0.0 \\
46\end{array}$ \\
\hline 9 & 0.500 & 0.5 & 0.0 & 0.3 & 0.33 & 0.33 & 0.0 & 0.0 & 0.2 & 0.7 & 0.1 & 0.66 & 0.2 & 0.0 & 0.0 & 0.9 & 0.1 & 0.0 & 0.9 & 0.1 & 0.0 & 0.0 \\
\hline
\end{tabular}




\begin{tabular}{|c|c|c|c|c|c|c|c|c|c|c|c|c|c|c|c|c|c|c|c|c|c|c|}
\hline \multirow{2}{*}{$\begin{array}{l}\text { Par } \\
\text { t. } \\
\text { no. }\end{array}$} & \multicolumn{3}{|c|}{ Level 2.1} & \multicolumn{4}{|c|}{ Level 2.2} & \multicolumn{4}{|c|}{ Level 3.1} & \multicolumn{4}{|c|}{ Level 3.2} & \multicolumn{3}{|c|}{ Level 3.3} & \multicolumn{3}{|c|}{ Level 3.4} & \multirow{2}{*}{$\begin{array}{l}\text { Avg } \\
\text { CR }\end{array}$} \\
\hline & $\begin{array}{l}\text { Tech./ } \\
\text { Eq. }\end{array}$ & $\begin{array}{l}\text { Pro } \\
\text { c. }\end{array}$ & CR & Eq. & $\begin{array}{l}\text { Tra } \\
\text { n. }\end{array}$ & $\begin{array}{l}\text { Con } \\
\text { t. }\end{array}$ & CR & OD & CD & $\mathbf{L F}$ & CR & $\begin{array}{l}\text { EW } \\
\mathbf{V}\end{array}$ & GV & IV & CR & EC & $\begin{array}{l}\mathbf{n E} \\
\mathrm{C}\end{array}$ & CR & DP & $\mathbf{n P}$ & CR & \\
\hline & & 00 & 00 & 33 & 3 & 3 & 00 & 51 & 27 & 22 & 80 & 3 & 78 & 58 & 46 & 00 & 00 & 00 & 00 & 00 & 00 & 38 \\
\hline 10 & 0.500 & $\begin{array}{l}0.5 \\
00\end{array}$ & $\begin{array}{l}0.0 \\
00\end{array}$ & $\begin{array}{l}0.7 \\
14\end{array}$ & $\begin{array}{l}0.14 \\
3\end{array}$ & $\begin{array}{l}0.14 \\
3\end{array}$ & $\begin{array}{l}0.0 \\
00\end{array}$ & $\begin{array}{l}0.0 \\
61\end{array}$ & \begin{tabular}{|l}
0.1 \\
76
\end{tabular} & $\begin{array}{l}0.7 \\
63\end{array}$ & $\begin{array}{l}0.0 \\
93\end{array}$ & $\begin{array}{l}0.76 \\
3\end{array}$ & $\begin{array}{l}0.1 \\
76\end{array}$ & $\begin{array}{l}0.0 \\
61\end{array}$ & $\begin{array}{l}0.0 \\
93\end{array}$ & $\begin{array}{l}0.8 \\
57\end{array}$ & $\begin{array}{l}0.1 \\
43\end{array}$ & $\begin{array}{l}0.0 \\
00\end{array}$ & $\begin{array}{l}0.8 \\
75\end{array}$ & $\begin{array}{l}0.1 \\
25\end{array}$ & $\begin{array}{l}0.0 \\
00\end{array}$ & $\begin{array}{l}0.0 \\
31\end{array}$ \\
\hline 11 & 0.889 & $\begin{array}{l}0.1 \\
11\end{array}$ & $\begin{array}{l}0.0 \\
00\end{array}$ & $\begin{array}{l}0.7 \\
96\end{array}$ & $\begin{array}{l}0.12 \\
5\end{array}$ & $\begin{array}{l}0.07 \\
9\end{array}$ & $\begin{array}{l}0.0 \\
46\end{array}$ & $\begin{array}{l}0.0 \\
56\end{array}$ & $\begin{array}{l}0.4 \\
63\end{array}$ & $\begin{array}{l}0.4 \\
81\end{array}$ & $\begin{array}{l}0.0 \\
01\end{array}$ & $\begin{array}{l}0.75 \\
1\end{array}$ & $\begin{array}{l}0.1 \\
78\end{array}$ & $\begin{array}{l}0.0 \\
70\end{array}$ & $\begin{array}{l}0.0 \\
25\end{array}$ & $\begin{array}{l}0.8 \\
89\end{array}$ & $\begin{array}{l}0.1 \\
11\end{array}$ & $\begin{array}{l}0.0 \\
00\end{array}$ & $\begin{array}{l}0.9 \\
00\end{array}$ & $\begin{array}{l}0.1 \\
00\end{array}$ & $\begin{array}{l}0.0 \\
00\end{array}$ & $\begin{array}{l}0.0 \\
12\end{array}$ \\
\hline $\begin{array}{l}\mathrm{Me} \\
\text { an }\end{array}$ & 0.527 & $\begin{array}{l}0.4 \\
36\end{array}$ & - & $\begin{array}{l}0.4 \\
89\end{array}$ & $\begin{array}{l}0.14 \\
9\end{array}$ & $\begin{array}{l}0.23 \\
1\end{array}$ & - & $\begin{array}{l}0.0 \\
66\end{array}$ & $\begin{array}{l}0.2 \\
22\end{array}$ & $\begin{array}{l}0.6 \\
73\end{array}$ & - & $\begin{array}{l}0.76 \\
8\end{array}$ & $\begin{array}{l}0.1 \\
55\end{array}$ & $\begin{array}{l}0.0 \\
70\end{array}$ & - & $\begin{array}{l}0.8 \\
90\end{array}$ & $\begin{array}{l}0.1 \\
09\end{array}$ & - & $\begin{array}{l}0.8 \\
90\end{array}$ & $\begin{array}{l}0.1 \\
09\end{array}$ & & - \\
\hline Avg & 0.535 & $\begin{array}{l}0.4 \\
65\end{array}$ & $\begin{array}{l}0.0 \\
00\end{array}$ & $\begin{array}{l}0.5 \\
39\end{array}$ & $\begin{array}{l}0.17 \\
4\end{array}$ & $\begin{array}{l}0.28 \\
7\end{array}$ & $\begin{array}{l}0.0 \\
10\end{array}$ & $\begin{array}{l}0.0 \\
66\end{array}$ & $\begin{array}{l}0.2 \\
48\end{array}$ & $\begin{array}{l}0.6 \\
86\end{array}$ & $\begin{array}{l}0.0 \\
63\end{array}$ & $\begin{array}{l}0.77 \\
0\end{array}$ & $\begin{array}{l}0.1 \\
60\end{array}$ & $\begin{array}{l}0.0 \\
71\end{array}$ & $\begin{array}{l}0.0 \\
57\end{array}$ & $\begin{array}{l}0.8 \\
90\end{array}$ & $\begin{array}{l}0.1 \\
10\end{array}$ & $\begin{array}{l}0.0 \\
00\end{array}$ & $\begin{array}{l}0.8 \\
90\end{array}$ & $\begin{array}{l}0.1 \\
10\end{array}$ & $\begin{array}{l}0.0 \\
00\end{array}$ & $\begin{array}{l}0.0 \\
24\end{array}$ \\
\hline $\begin{array}{l}\text { Std. } \\
\text { dev. }\end{array}$ & 0.117 & $\begin{array}{l}0.1 \\
17\end{array}$ & $\begin{array}{l}0.0 \\
00\end{array}$ & $\begin{array}{l}0.2 \\
36\end{array}$ & $\begin{array}{l}0.10 \\
0\end{array}$ & $\begin{array}{l}0.17 \\
4\end{array}$ & $\begin{array}{l}0.0 \\
22\end{array}$ & $\begin{array}{l}0.0 \\
09\end{array}$ & $\begin{array}{l}0.1 \\
37\end{array}$ & $\begin{array}{l}0.1 \\
32\end{array}$ & $\begin{array}{l}0.0 \\
44\end{array}$ & $\begin{array}{l}0.04 \\
1\end{array}$ & $\begin{array}{l}0.0 \\
46\end{array}$ & $\begin{array}{l}0.0 \\
10\end{array}$ & $\begin{array}{l}0.0 \\
42\end{array}$ & $\begin{array}{l}0.0 \\
12\end{array}$ & $\begin{array}{l}0.0 \\
12\end{array}$ & $\begin{array}{l}0.0 \\
00\end{array}$ & $\begin{array}{l}0.0 \\
11\end{array}$ & $\begin{array}{l}0.0 \\
11\end{array}$ & $\begin{array}{l}0.0 \\
00\end{array}$ & $\begin{array}{l}0.0 \\
12\end{array}$ \\
\hline
\end{tabular}


A.1. Acronyms of available options for the life cycle phases

$\mathrm{CD}$

DP

controlled dump

detailed procedures

DS

dry sterilisation/disinfection

$\mathrm{E}$

encapsulation

EC

engineered container

$\mathrm{EC} \rightarrow \mathrm{EC}$

from engineered container to engineered container

$\mathrm{EC} \rightarrow \mathrm{nEC}$

from engineered container to non-engineered container

EL

EnW

engineered location

EP

engineered non-wheeled

ES

EW

engineered pit

engineered storage

engineered wheeled

EWT

engineered wheeled transport

EWV

engineered wheeled vehicle

GT

GV

general transport

IT

general vehicle

IV

inappropriate transport

inappropriate vehicle

LF

landfill

$\mathrm{nEC}$

non-engineered container

$\mathrm{nEC} \rightarrow \mathrm{EC}$

$\mathrm{nEC} \rightarrow \mathrm{nEC}$

from non-engineered container to engineered container

from non-engineered container to non-engineered container 


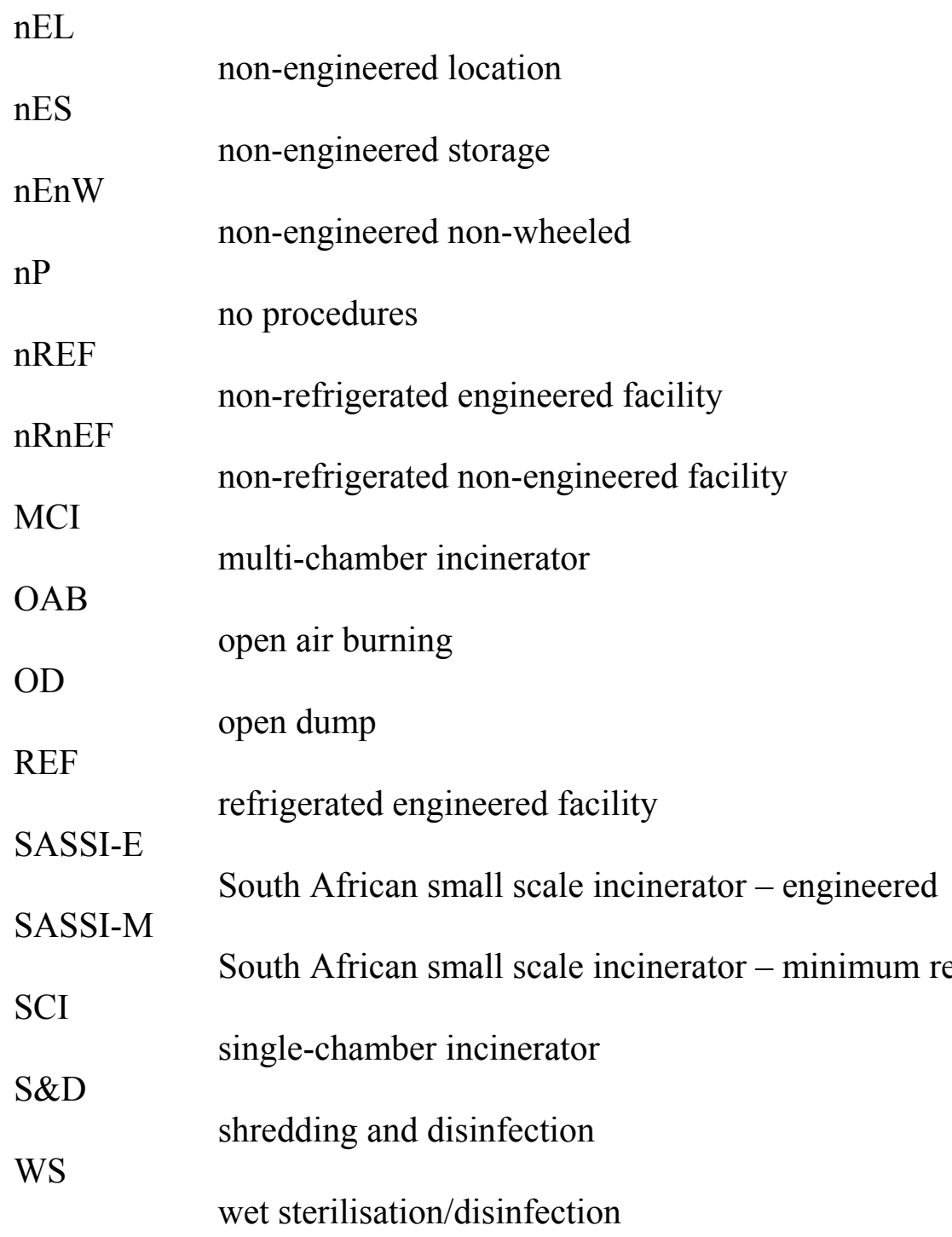


Corresponding author. Tel.: +27 12420 3929; fax: +27 123625307 .

${ }^{1}$ Consistency is a statistical measure of the extent to which an individual's decision structure, i.e. set of assessment judgements, is closer to being logically related than randomly chosen. The consistency of judgements reflects the extent to which the decision-maker(s) understands the problem, is knowledgeable of the decision variables involved, understands the assessment process, and is able to make a series of logically related judgements based on uncertain and often incomplete information (Noble, 2004). ${ }^{2}$ Sustainable development has been defined as - development that meets the needs of the present without compromising the ability of future generations to meet their own needs (Bruntland, 1987).

${ }^{3}$ Agenda 21 is the report of the United Nations Conference on Environment and Development held in Rio in 1992 at which consensus was reached on the principles of continuous improvement and a systems approach to waste management (UN, 1992). ${ }^{4}$ Seventy five percentage of deaths in sub-Saharan developing countries are reportedly due to infectious disease compared with $10 \%$ in the developed countries (WHO, 2004). ${ }^{5}$ As described in Chapter 34 of Agenda 21 (UN, 1992).

${ }^{6}$ Primary health care facilities, in the developing country context, are defined as facilities at and from which a range of primary health care services are provided, but inpatient services are typically not provided. It is normally open only 8 hours a day. Certain staff may, however, be required to sleep at or near the clinic so that they are available on call in case of emergency (SA DoH, 1998). 